$1 / 1968 \delta 1$
SANDIA REPORT

SAND96-8224 • UC-406

Unlimited Release

Printed July 1996

\title{
Simulation of Surface Roughness during the Formation of Thermal Spray Coatings
}

Michael Kanouff

Prepared by

Sandia National Laboratories

Albuquerque, New Mexico 87185 and Livermore, California 94551

for the United States Department of Energy

under Contract DE-AC04-94AL85000

Approved for public release; distributionits unlimited.

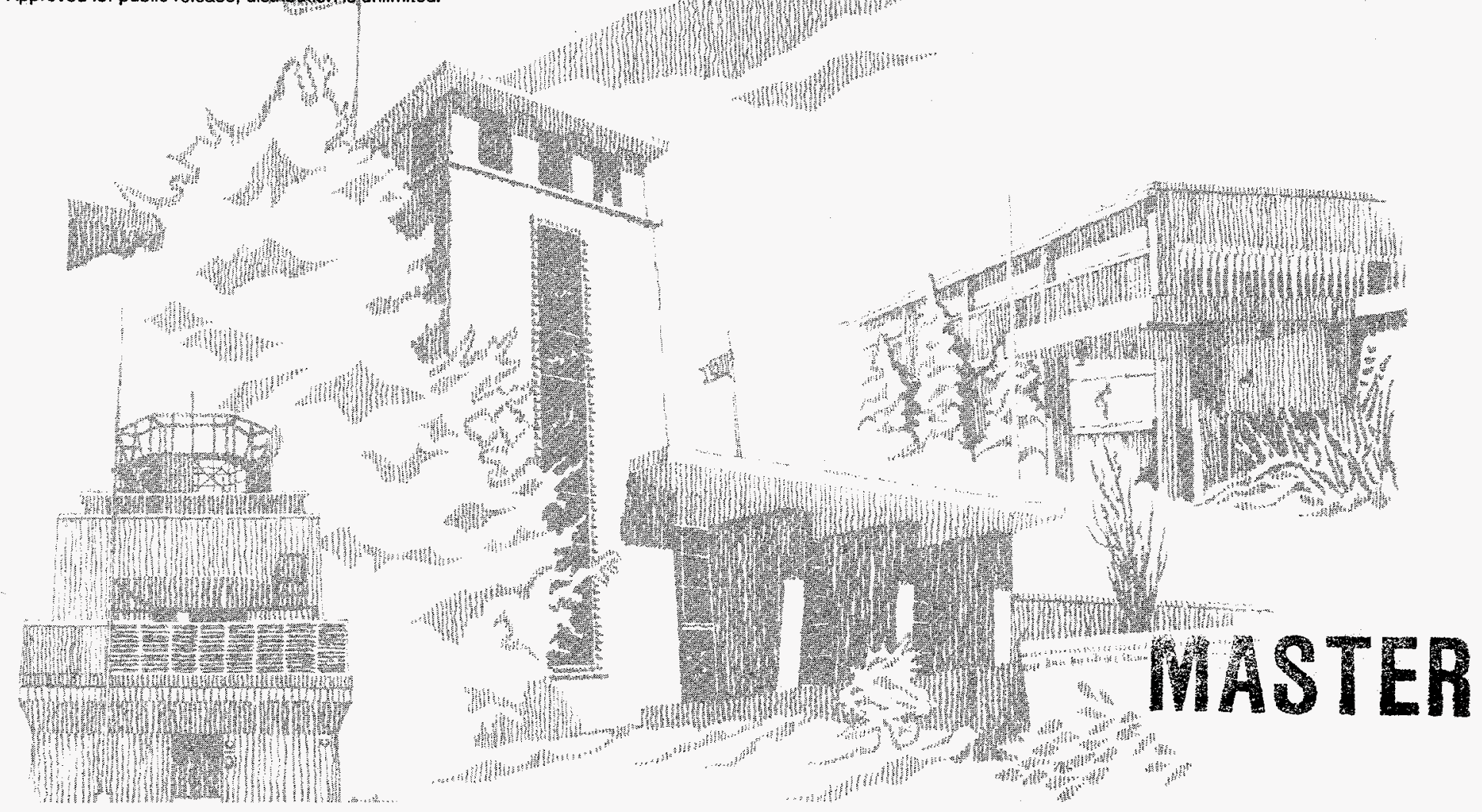


Issued by Sandia National Laboratories, operated for the United States Department of Energy by Sandia Corporation.

NOTICE: This report was prepared as an account of work sponsored by an agency of the United States Government. Neither the United States Government nor any agency thereof, nor any of their employees, nor any of the contractors, subcontractors, or their employees, makes any warranty, express or implied, or assumes any legal liability or responsibility for the accuracy, completeness, or usefulness of any information apparatus, product, or process disclosed, or represents that its use would not infringe privately owned rights. Reterence nerein to any specific commercial product, process, or service by trade name, trademark, manufacturer, or otherwise, does not necessarily constitute or imply its endorsement, recommendation, or favoring by the United States Government, any agency thereof or any of their contractors or subconractors. The views and opinions expressed herein do not necessarily state or reflect those of the United States Government, any agency thereof or any of their contractors or subcontractors. 
SAND96-8224

Unlimited Release

Printed July 1996

\title{
SIMULATION OF SURFACE ROUGHNESS DURING THE FORMATION OF THERMAL SPRAY COATINGS
}

\author{
M. P. Kanouff \\ Mechanics and Simulation of Manufacturing Processes Department \\ Sandia National Laboratories \\ Livermore, CA 94550
}

\begin{abstract}
The formation of a thermal spray coating has been analyzed to identify methods to reduce the surface roughness of the coating. In developing the analysis, a new methodology was developed. The method uses a string of equally spaced node points to define the shape of the coating surface and to track the change in this shape as the thermal spray mass is deposited. The method allows for the calculation of arbitrary shapes for the coating surface which may be very complex. The model simulates the stochastic deposition of a large number of thermal spray droplets, where experimental data is used for the mass flux distribution on the target surface. This data shows that when the thermal spray mass impinges on the target surface a large fraction of it, called over-spray, splashes off the target and is re-deposited with a small spray angle. This component of the deposited mass results in a large coating roughness. The analysis was used in a parameter study to identify methods for reducing the coating roughness. The effect of the shape of the profile for the preroughened substrate was found to be small. Decreasing the droplet size by a factor of two decreased the roughness by $13 \%$. Increasing the spray angle for the over-spray by a factor of two decreased the roughness by $50 \%$, and decreasing the amount of over-spray by a factor of two decreased the roughness by $51 \%$.
\end{abstract}




\section{ACKNOWLEDGMENT}

The author wishes to thank the Sandia and General Motors personnel who were members of this research and development team, including; Dr. W. L. Oberkampf, Org. 9115, for his guidance and encouragement during the development of the coating formation model; Dr. R. A. Neiser, Dr. M. F. Smith and Mr. T. J. Roemer, all Org. 1841, for the creative experimental techniques they used to provide the data which lead to a more realistic model; Dr. R. C. Dykhuizen, Org. 9113, and Dr. R. Teets, GM R\&D, for their ideas for some of the physical phenomena included in the model; and to Mr. M. Kramer and Mr. L. Byrnes, both GM Powertrain, for the education they provided to me on the High Velocity Oxygen-Fuel thermal spray process.

The author would also like to thank Professor R. Greif, UC Berkeley, for his extensive editing of this manuscript. Finally, the author wishes to thank Dr. M. L. Callabresi, Org. 8743, for the confidence he showed when he provided the author with the opportunity to work on this most challenging and interesting program.

This work was funded through a Cooperative Research and Development Agreement between Sandia National Laboratories and the General Motors Corporation. 


\section{DISCLAIMER}

Portions of this document may be illegible in electronic image products. Images are produced from the best available original document. 


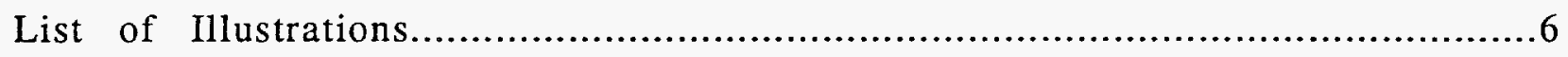

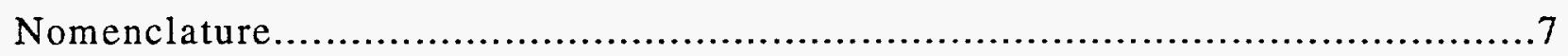

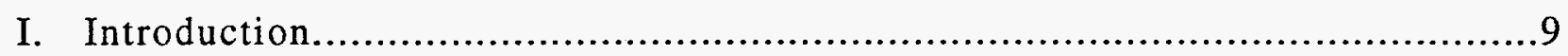

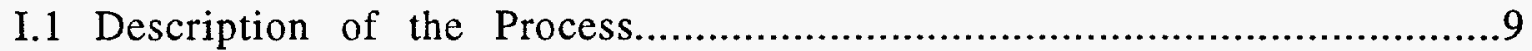

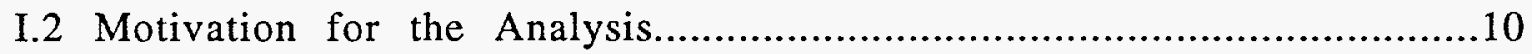

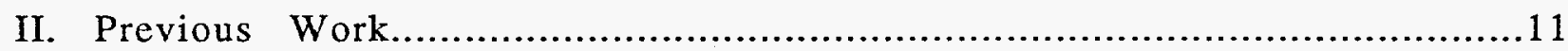

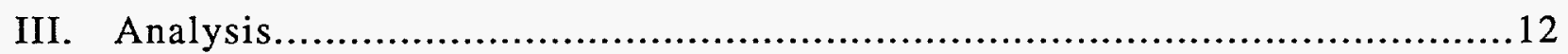

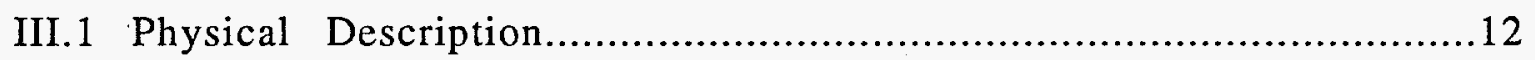

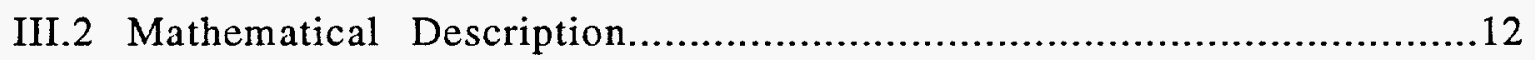

III.2.1 The Thermal Spray Mass Flux Distributions..................................13

III.2.2 Describing the Mass Flux in Terms of Droplets..............................16

III.2.3 Shading ........................................................ 17

III.2.4 Droplet Spreading................................................18

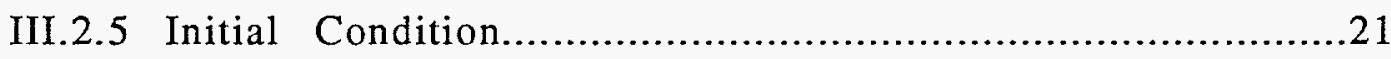

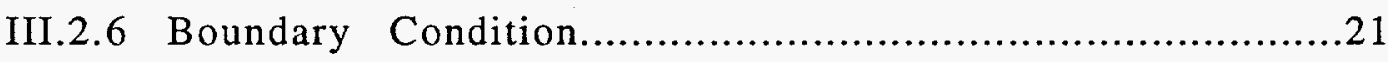

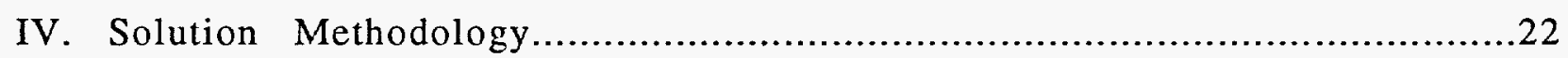

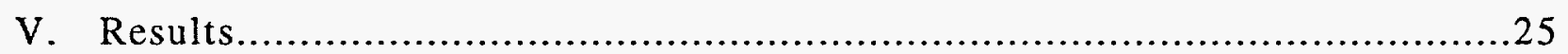

V.1 Continuous Mass Deposition on Simple Surfaces.......................................26

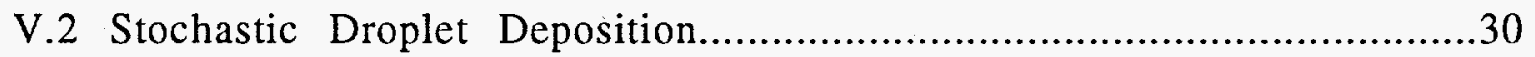

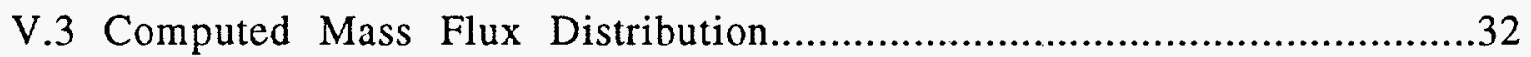

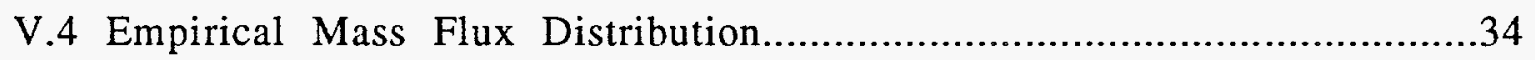

V.4.1 Comparison with Experimental Data.............................................36

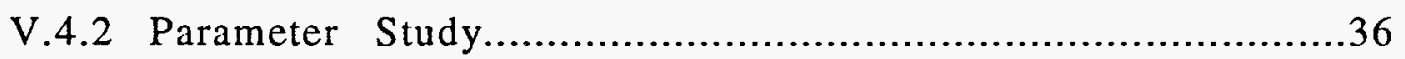

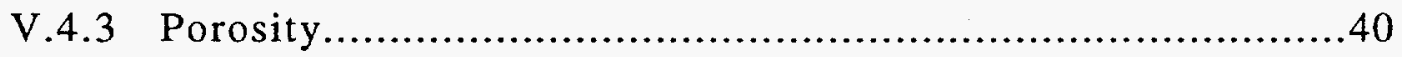

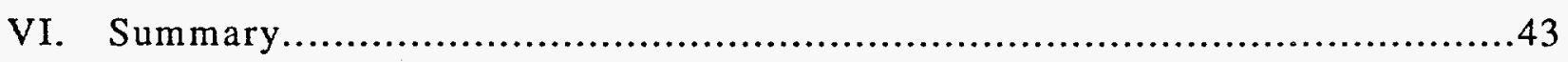

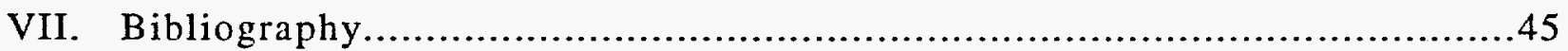

Appendix A. The Thermal Spray Mass Flux Distribution..........................................

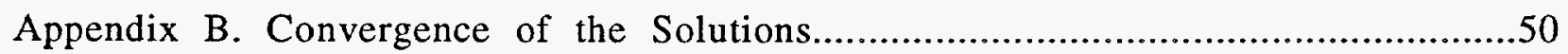




\section{ILLUSTRATIONS}

No.

1. A schematic diagram of the HVOF process for coating engine cylinder bores...................9

2. The coordinate system used for the coating formation analysis...............................12

3. A schematic diagram of the mass flux and coating profile........................................13

4. An infra-red image of the HVOF thermal spray impinging on a flat plate........................14

5. The mass flux distributions for an HVOF thermal spray jet impinging on a flat plate..........15

6. A schematic diagram for the position of the target ............................................15

7 A schematic diagram showing the division of the droplet mass flux field into bins............16

8. A schematic diagram of a streamline and droplet impact point on the coating surface ..........18

9. The data by Madejski (1976) for the splat length as a function of impact angle.................19

10. A schematic diagram showing the splat location relative to the droplet impact point ..........20

11. A schematic diagram of the node point distribution along the coating surface...................22

12. A schematic diagram of erroneous loops in the computed coating surface......................23

13. A schematic diagram of the computed coating surface after removal of a loop..................24

14. A schematic diagram of the coating surface and the arrangement of peaks and valleys.......25

15. The coating surface for $L=400, \theta=26^{\circ}$ and $S_{m} 0=1000$ (Stable Scallop Regime).............26

16. The four regimes for scallop growth in the input parameter space..............................27

17. The coating surface for $L=400$ (a) $\theta=15^{\circ}, S_{m 0}=700$ (b) $\theta=45^{\circ}, S_{m 0}=1000$ (Stable Rg.).27,28

18. The coating surface for $L=230, \theta=26^{\circ}$ and $S_{m} 0=750$ (Bifurcating Scallop Regime)........28

19. The coating surface for $L=550, \theta=26^{\circ}$ and $S_{m 0}=400$ (No Scallop Regime)..................28

20. The coating surface for $L=300, \theta=20^{\circ}$ and $S_{m 0}=300$ (Merging Scallop Regime)............29

21. The effect of pre-roughness elements with non-uniform amplitudes (Stable Scallop Reg.)...30

22. The coating surface for stochastic droplet deposition on a smooth substrate ...................31

23. The coating roughness for stochastic droplet deposition on smooth and rough substrates ...31

24. The coating surface for the calculated mass flux distribution......................................33

25. A comparison of the calculated and measured coating surface profiles...........................34

26. The coating roughness for the empirical mass flux distribution..............................35

27. Comparison of calculated and measured results for the coating roughness ......................36

28. The effect of the pre-roughness profile shape on the coating roughness.........................37

29. The effect of the droplet size on the coating roughness..........................................38

30. The effect of the splash fraction on the coating roughness........................................39

31. The effect of the spray angle for the over-spray on the coating roughness.......................40

32. The shape of a large pore which forms in a hole in the substrate...............................41

33. The shape of a large pore which forms in a hole in the substrate (second example)...........42

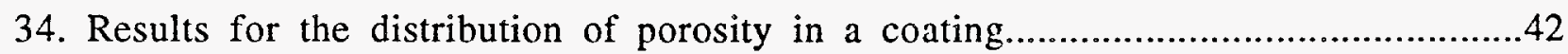

35. The effect of modifications to the model parameters on the coating roughness..................44

36. A conceptual design for a spray gun for increasing the spray angle for the over-spray.........44 


\section{NOMENCLATURE}

A

$\mathrm{Ab}$

D

$\mathrm{D}_{\mathrm{m}}$

$D_{S}$

f

$\mathrm{f}_{\mathrm{S}}$

$\mathrm{g}$

HVOF

i

j

L

m

$\mathrm{m}$

$\mathrm{N}_{\mathrm{D}}$

n

$\mathrm{R}_{\mathrm{a}}$

$\mathrm{R}_{\mathrm{a} 0}$

$\mathrm{Re}$

$\mathrm{R}_{\mathrm{n}}$

$\mathbf{r}$

$\mathrm{S}_{\mathrm{m}}$

$\mathrm{S}_{\mathrm{m} 0}$

$T$

$\mathbf{t}$

$\mathrm{V}_{\mathrm{D}}$

W

$w_{i}$

$\mathbf{X}$

$\mathrm{x}_{\mathrm{S}}$

y

Amplitude of a sine wave

Cross sectional area of a mass flux bin measured perpendicular to the direction of the mass flux

Droplet size

Droplet size in the main spray

Droplet size in the over-spray

Fraction of a splat located 'up-stream' of the droplet impact point Splash fraction (fraction of the mass deposited by the over-spray) Rate of accumulation of mass at a point on the coating surface per unit area

Acronym for High Velocity Oxygen Fuel thermal spray process

Unit vector in the $\mathrm{x}$-direction

Unit vector in the $y$-direction

Length of a splat

Thermal spray mass flux vector

Thermal spray mass flux magnitude

Droplet number density distribution at the exit of a thermal spray gun

Unit vector locally perpendicular to the coating surface

Roughness variable quantifying the dimension of the surface roughness elements perpendicular to the substrate

Initial value for $\mathrm{R}_{\mathrm{a}}$

Reynolds number

Random number

Position vector for a point on the coating surface

Roughness variable quantifying the dimension of the surface roughness elements parallel to the substrate

Initial value for $S_{m}$

Thickness of a splat

Time

Volume of a droplet

Width of a splat

Fraction of the thermal spray mass deposited in mass flux zone, $\mathrm{i}$

Cartesian coordinate parallel to the substrate

Constant defining a streamline

Cartesian coordinate perpendicular to the substrate 


$\mathrm{y}_{\mathrm{m}}$
$\mathrm{zg}_{\mathrm{g}}$
$\beta$
$\chi$
$\delta \mathrm{t}$
$\delta \mathrm{t}_{\mathrm{a}}$
$\delta \mathrm{t}_{\mathrm{m}}$
$\gamma$
$\theta$
$\theta_{\mathrm{s}}$
$\rho$
$\tau$
$\xi^{\prime}$
$\xi^{\prime}$

Subscripts:

b

i

k

\section{Superscipts:}

$\mathrm{n}$
Average value of $y$ for the coating surface

Initial position of a droplet in the exit plane of the thermal spray gun

Local impact angle of a droplet on the coating surface

$\mathrm{x}$-component of $\tau$

Time interval between droplet impacts

Inverse of the average value of $1 / \delta \mathrm{t}$

Average value of $\delta t$

y-component of $\tau$

Thermal spray angle

Spray angle of the over-spray

Density of the thermal spray material

Unit vector locally tangent to the coating surface

Spread coefficient for a perpendicular droplet impact

Spread coefficient for a non-perpendicular droplet impact

Value for the mass flux bin, $\mathrm{b}$

Value for the mass flux zone, $i$

Value for the node point, $k$

Value for the time step, $n$ 


\section{INTRODUCTION}

\section{I.1 Description of the Process}

Thermal spraying refers to a family of processes used to apply coatings on surfaces for providing enhanced protection from wear, corrosion, or thermal damage. In a cooperative research and development agreement (CRADA), GM and Sandia National Laboratories developed a thermal spray process, called High-Velocity Oxygen-Fuel (HVOF), for applying a wear resistant coating to the surfaces of aluminum engine cylinder bores [Byrnes and Kramer, 1994]. Figure 1 shows a schematic of the process hardware. Fuel (methane), oxygen and air are supplied to the HVOF gun, where the fuel and oxygen react in a chamber called an air cap near the exit of the gun [Hassan, Oberkampf, Neiser and Roemer (1995)]. The combustion process creates high gas temperatures and pressures in the air cap. The coating material, steel, is fed into the gun in the form of wire. The wire travels into the air cap and is melted by the high gas temperatures. The high gas pressure in the air cap accelerates the gas to high velocities, which flows over the wire and strips the molten steel off of it [Neiser, Brockmann, O'Hern, Dykhuizen, Smith, Roemer and Teets (1995)]. The gas velocity atomizes the molten steel, i.e. breaks it up into small droplets, which are accelerated and transported to the target surface where they are deposited. As illustrated in Figure 1, the steel spray exits the gun and impinges on the surface of the cylinder bore with an off-normal angle. The HVOF gun rotates rapidly and strokes vertically along the axis of the cylinder bore to attain uniform deposition of the steel.

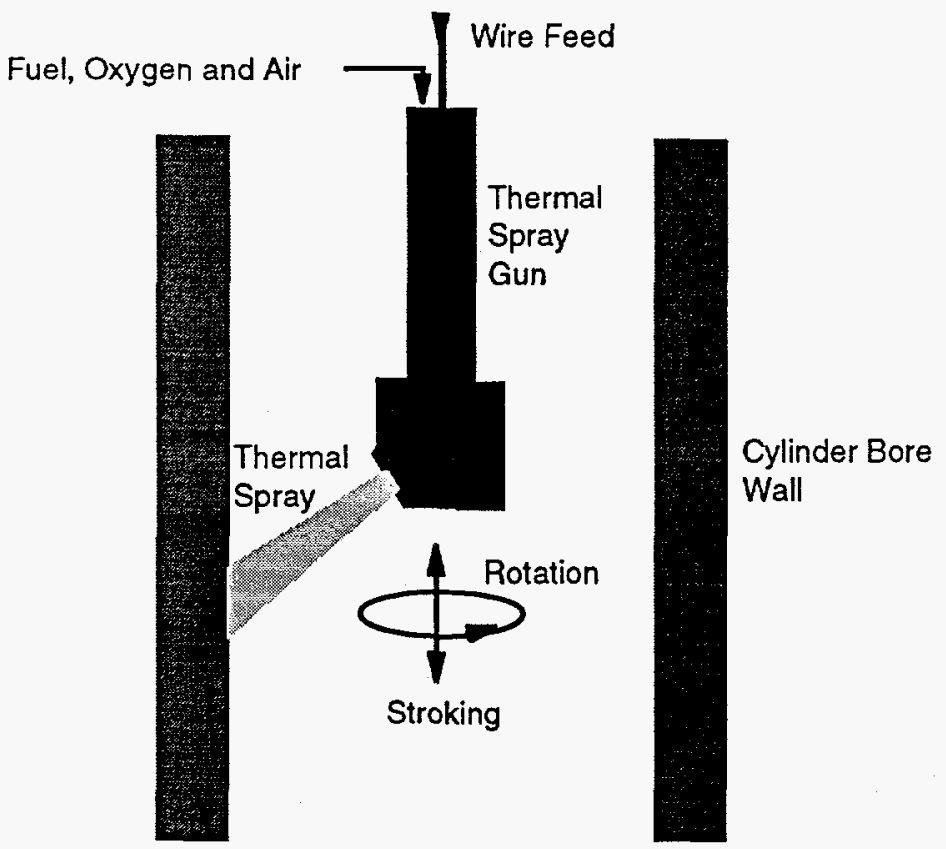

Figure 1. A schematic of the HVOF process for coating engine cylinder bores.

When the droplets strike the cylinder surface they deform into small flat disk shaped 'splats'. The heat in the splat rapidly conducts into the massive cylinder wall and the splat solidifies. The solidification process occurs over a period of micro-seconds, and each splat is well solidified before another droplet is deposited on top of it. The rapid solidification of the splat results in very small grain sizes in the solidified steel which makes it hard and gives it good wear resistant 
properties. The surface of the cylinder bore is pre-roughened before coating to promote good bonding with the thermal sprayed coating.

\section{I.2 Motivation for the Analysis}

One problem with the process is that the coating obtained has a very rough surface. A smooth surface is required because the engine piston rings must slide upon this surface and maintain a good seal between the cylinder bore surface and ring to contain the high pressure gases created during engine operation. Consequently, a machining operation must be added to the overall production procedure to obtain a smooth coating surface. This increases the time and cost of the production procedure. Moreover, machining the coating is difficult due to the hardness of the material, which results in short lifetimes for the machine tools and increases the time and cost of the machining operation.

The purpose for developing the analytical model is to help identify methods for creating smoother coatings during spraying. In order to be a predictive tool, the model must reproduce the roughness observed in the coating. This will reveal the phenomena which contribute to the roughness formation. Then, the model can be used to investigate the effects of modifications to the process on the resulting coating. This will quantify the magnitude and scope of the modifications required with respect to the resulting reductions in coating roughness that are obtained. 


\section{PREVIOUS WORK}

Cirolini, Harding and Jaccuci (1991) studied the formation of a plasma sprayed thermal barrier coating for the purpose of calculating the porosity in the coating. They calculated the coating shape in two-dimensions for thermal spray droplets impacting on a pre-roughened substrate surface at a normal angle. Experimental data were used for the thermal spray droplet size, droplet impact velocity and droplet temperature distributions. They simulated the stochastic nature of the droplet deposition process when the droplets impact on the substrate in a random order. A rectangular mesh was constructed in the space above the substrate where the coating formed which divided the space into a large number of small voids. As each droplet was deposited in the simulation, the location of the resulting splat was recorded in the appropriate voids. The size of a splat was related to the size and Reynolds number of the impacting droplet (based on work by Madejski, 1976). They assumed that the central portion of the splat adhered to the underlying target surface, but the outer portion of the splat was assumed to curl up based on calculations for the temperature difference across the splat and the resulting thermal stresses. They also assumed that curled up splats could be 'hammered' back down onto the underlying target surface if they experienced a direct impact from another droplet. Although the primary purpose was not to compute surface roughness, their results showed that the roughness of the coating surface was larger than the pre-roughness of the substrate. They later extended their work to study the effects of rastering the thermal spray gun on coating porosity (Mulheran, Harding, Kingswell and Scott, 1992, and Harding, Mulheran, Cirolini, Marchese and Jaccuci, 1995). They did not investigate the effect of process parameters on the coating roughness.

Knotek, Lugscheider, Jokiel, Schnaut and Wiemers (1994) modeled the formation of a chromium coating as applied with a powder fed HVOF process. They calculated the droplet temperatures and velocities for input to their coating formation model, where they calculated the two-dimensional shape of the coating. They simulated the stochastic deposition of the droplets onto a pre-roughened substrate with a normal angle of incidence. The size of a splat resulting from a droplet impact was related to the size and Reynolds number of the impacting droplet (based on the work of Madejski, 1976). Their results also showed that the roughness on the coating surface was larger than the roughness of the substrate, although they did not emphasize this aspect of their results.

There appears to be very little work done in the form of modeling the formation of rough surfaces on thermal spray coatings. The work cited above describe models which compute the shape of the coating surface, and therefore its roughness, but this was not their primary purpose. Studies of the effects of model and process parameters on the roughness of thermal spray coatings were not found. 


\section{ANALYSIS}

\section{III.1 Physical Description}

A model for the formation of a thermal spray coating has been developed for calculating the shape and roughness of the coating surface. The characteristics of the thermal spray mass flux are inputs to the model. The model assumes the process is two-dimensional, in the $x-y$ coordinate plane as shown in Figure 2. The $\mathrm{x}$-coordinate is parallel to the substrate surface (parallel to the axis of the
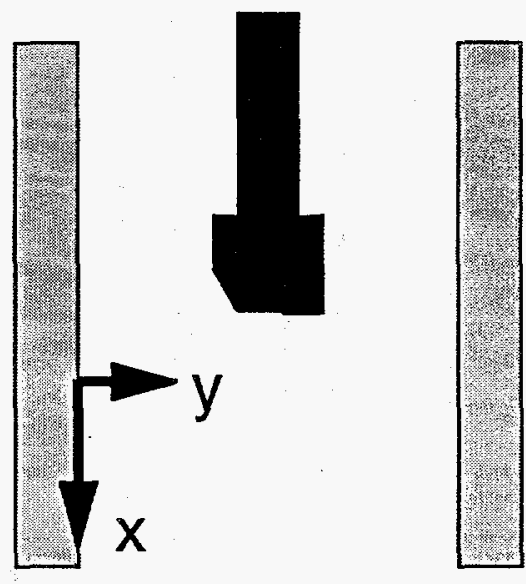

Figure 2. The coordinate system used for the coating formation analysis.

cylinder bore), and the y-coordinate is normal to the substrate surface. The effects of surface curvature in the azimuthal direction of the cylinder bore are ignored which is valid for coating thicknesses which are small compared to the radius of the cylinder bore. Only a small fraction of the axial length of the cylinder bore is modeled. This should be a good assumption as long as the length of cylinder bore included in the model is large compared to the coating thickness.

\section{III.2 Mathematical Description of the Process}

Equation (1) describes the rate of movement of a point on the coating surface due to the rate at which the thermal spray mass accumulates per unit area of the surface, g, divided by the density

$$
\frac{\mathrm{d} \mathbf{r}}{\mathrm{dt}}=\frac{\mathrm{gn}}{\rho}
$$

of the sprayed material, $\rho . \mathbf{r}$ is a position vector for the point $(\mathbf{r}=\mathrm{x} \mathbf{i}+\mathrm{y} \mathbf{j})$ which specifies its $\mathrm{x}$ and $\mathrm{y}$ coordinates, and $\mathbf{n}$ is the unit vector (dimensionless) locally normal to the coating surface (see Figure 3). Equation (1) states that a point on the coating surface moves in the direction of $\mathbf{n}$. The quantity, g, depends on the thermal spray mass flux characteristics, the degree of spreading that a droplet undergoes when it impacts on the surface, and the shading that may take place when a part of the coating surface shades another part of the surface thereby preventing the direct impact of 
thermal spray mass on the surface. The thermal spray mass flux vector, $m$, is characterized by its magnitude, $m$, with units of mass per unit time per unit area, and its direction described in terms of the angle, $\theta$, as defined in Figure 3 . In addition, the droplets in the thermal spray have a diameter, $D$. When $g$ is determined and Equation (1) is solved for each and every point on the coating surface, a complete description of the evolution of the shape and position of the surface is obtained.
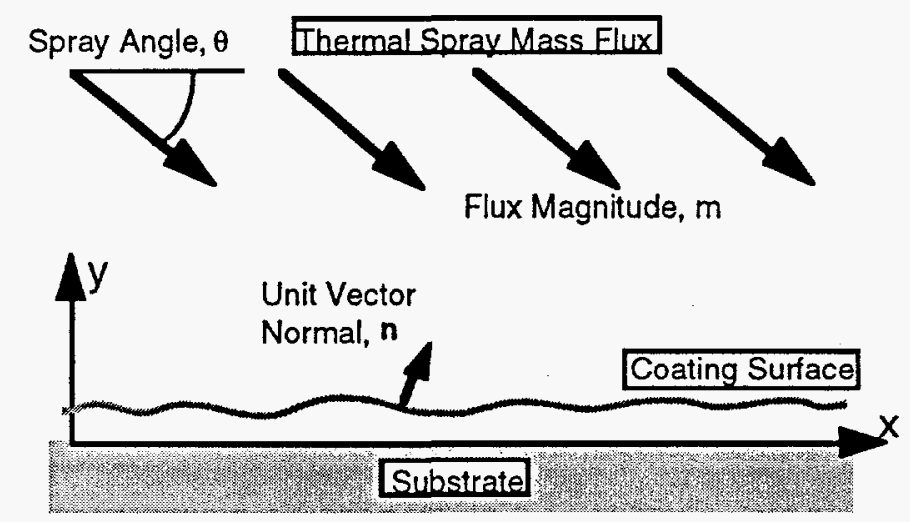

Figure 3. A schematic diagram of the mass flux and coating profile.

\section{III.2.1 The Thermal Spray Mass Flux Distributions}

The values of $\mathrm{m}, \theta$ and $\mathrm{D}$ characterizing a thermal spray are difficult to obtain. Some numerical simulations have been published, but many of these did not include the target in the simulations, which can alter the gas flow field and droplet trajectories. Domnick, Lindenthal, Mundo, Ruger and Sommerfeld (1994) computed the trajectories of water droplets transported by a gas jet impinging on a flat plate at a normal angle. The trajectories of the water droplets were all very close to one another and nearly straight and parallel with the axis of the gas jet in the region remote from the target. As the target was approached, a significant number of the droplets smaller than 20 microns turned with the gas flow field and traveled parallel to the target. Lopez and Oberkampf (1995) computed the transport of molten metal droplets by an axi-symmetric gas jet impinging on a flat plate at a normal angle. They considered droplets as small as 10 microns and all were found to impact on the plate. Some of the smaller droplets turned a small amount as the plate was approached, but all were found to impact on the plate within $25^{\circ}$ of the normal angle. In contrast to water droplets, the larger density of the molten metal droplets results in a large inertial force which prevents the metal droplets from turning and missing the target as the water droplets did in the calculations of Domnick et al. (1994).

An experiment was carried out to study a thermal spray impinging on a flat plate at an angle. The experiment is described in Appendix A. The thermal spray gun used to coat the cylinder bore was used to make a deposit on a flat plate, where the plate was held parallel to the axis of the gun and at a distance from the gun corresponding to the radius of the engine cylinder bore. The gun did not stroke and it did not rotate. Figure 4 shows an infra-red video image of the thermal spray as it impinged on the plate. The image of the main spray coming from the gun can be seen as a narrow, slightly diverging cone as it approaches the plate. It remains approximately straight, i.e. there is not a lot of curvature in the image in agreement with the results of Lopez et al. (1995). The area over which the main spray impacts the target plate is approximately $15 \mathrm{~mm}$ long. Starting from the main spray impact zone, a very bright elongated region parallel to the plate can be seen which extends approximately $5 \mathrm{~mm}$ above the plate and is $70 \mathrm{~mm}$ long. This bright region appears to be due to 
splashing of the thermal spray droplets as they impact on the plate, where the splash product, called over-spray, is ejected off the plate surface and is swept downstream parallel to the plate (positive $\mathrm{x}$-direction) by the gas flow. The location and length of this bright region corresponds to the location and length of the mass deposit which was later found on the plate. Thus, while the main thermal spray jet impacts on the plate over an area that is only $15 \mathrm{~mm}$ long, the mass is redistributed over an area that is $75 \mathrm{~mm}$ long, where the redistribution appears to be due to splashing.

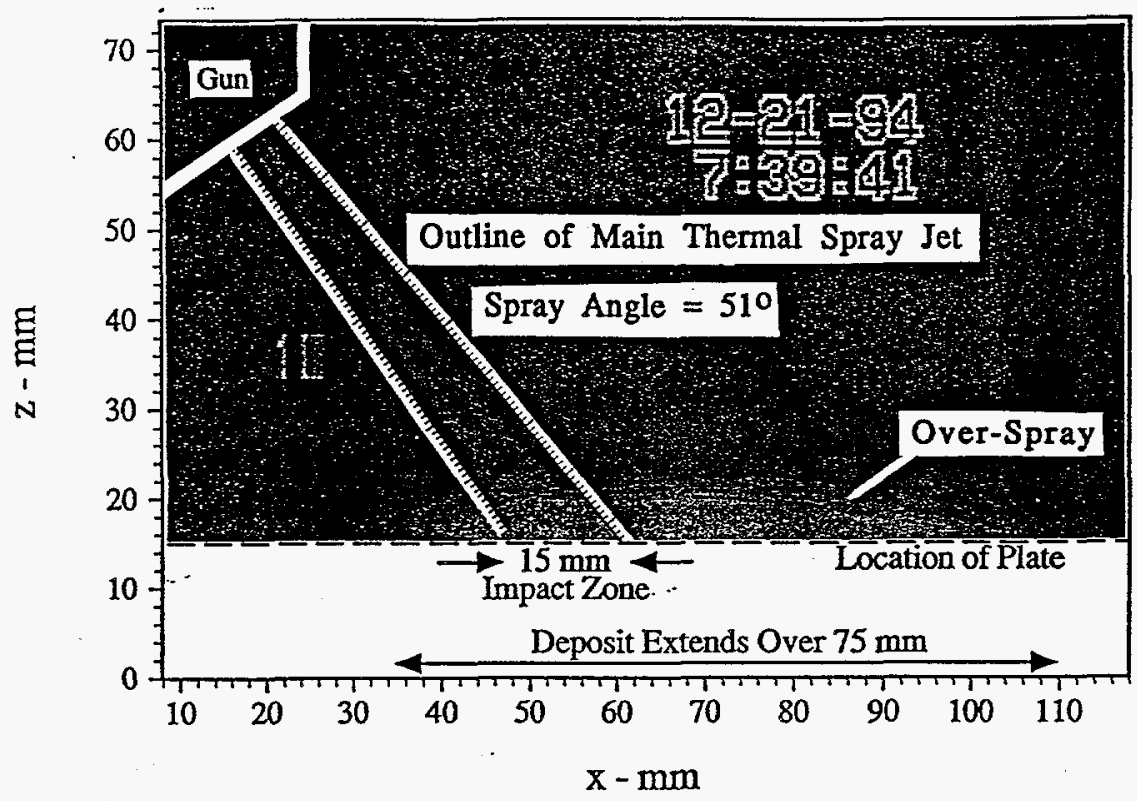

Figure 4. An infra-red image of the HVOF thermal spray impinging on a flat plate.

As discussed in Appendix A, the portion of the mass deposited away from the main spray impact zone was in the form of isolated columns of material that are separated by large spaces. This structure is due to a very small spray angle, $\theta$. As discussed later in this report, small spray angles can result in a large coating roughness. This makes it very important to quantify the deposition conditions of the over-spray, and include it in the thermal spray mass flux used for the roughness calculations. The deposit on the flat plate was divided into six zones; over each zone the deposit structure was approximately uniform. One of the zones coincides with the main spray impact zone, and five other zones are used to span the over-spray portion of the deposit. Appendix A describes the calculations which were carried out to obtain estimates for the mass flux magnitude, $m$, the spray angle, $\theta$, and droplet size, $\mathrm{D}$, within each zone; i.e. $\mathrm{m}_{\mathrm{i}}, \theta_{\mathrm{i}}$ and $\mathrm{D}_{\mathrm{i}}$. These results provide an estimate for the distributions of $\mathrm{m}, \theta$ and $\mathrm{D}$ over the target surface of both the main thermal spray jet and the over-spray, and are shown in Figure 5. The abscissa in Figure 5, $x$ ', is a coordinate parallel to the target surface and measured in a reference frame which, in general, must be considered to move with the thermal spray gun. The mass flux magnitude is expressed in terms of the fraction of the total mass deposit, $w_{i}$, that is deposited within each zone $i$, and is related to $m_{i}$ by Equation (2) (the subscript $i$ in Equation (2) refers to the zone number specified in Figure 5). These distributions show that most of the mass is deposited in the main spray impact zone (zone 2) where $\theta$ and $D$ are relatively large, and that a significant amount of the mass is deposited in the over-spray zones (zones 1 and 3-6) where $\theta$ and D are small. Zone 1 represents the part of the over-spray which is deposited 'up-stream' of the main deposit. The spray angle is measured 
between the direction of the positive $x$-axis and the mass flux vector, as shown in Figure 3 . The spray angle for zone $1,178^{\circ}$, is small in the sense that the angle with the negative $\mathrm{x}$-axis is just $2^{\circ}$.

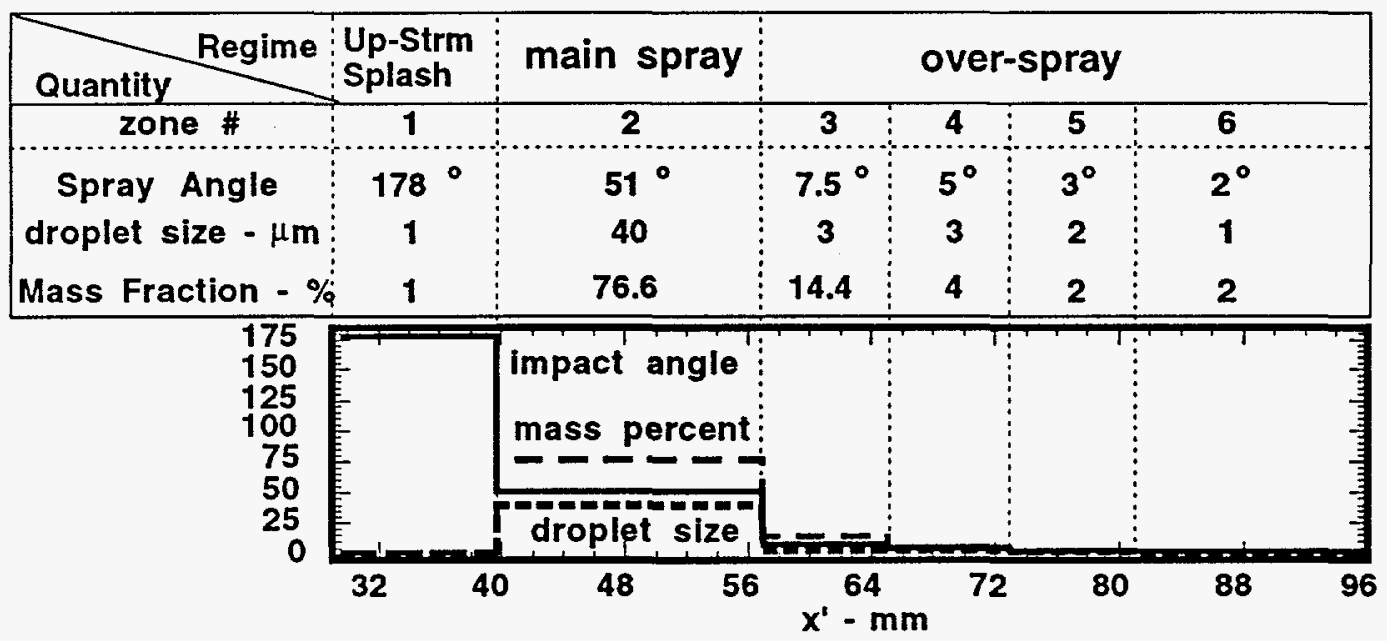

Figure 5. The distributions for the mass deposition rate, spray angle and droplet size in a HVOF thermal spray jet impinging on a flat plate.

$$
\mathrm{w}_{\mathrm{i}}=\frac{\mathrm{m}_{\mathrm{i}} \sin \theta_{\mathrm{i}} \Delta \mathrm{x}_{\mathrm{i}}{ }^{\prime}}{\sum_{\mathrm{i}=1}^{6} \mathrm{~m}_{\mathrm{i}} \sin \theta_{\mathrm{i}} \Delta \mathrm{x}_{\mathrm{i}}{ }^{\prime}}
$$

A uniform coating thickness is obtained on the cylinder bore by moving the thermal spray gun with constant speed along the axis of the bore while the bore is held stationary. In an alternative reference frame, the gun may be considered to be stationary while the bore moves. In this reference frame, each point on the bore surface moves relative to the gun such that it is exposed, sequentially, to all (six) of the mass flux zones shown in Figure 5. Recall that the length of the target included in the analysis represents only a small fraction of the total cylinder bore. If the position of this small fraction of the bore relative to the gun is, $\mathrm{X}^{\prime}$, then the relative motion between the model target and the gun is described as a time dependent value for $\mathrm{X}^{\prime}$, as shown in Figure (6). The gun stroke speed is assumed to be constant. A specific value for time gives a

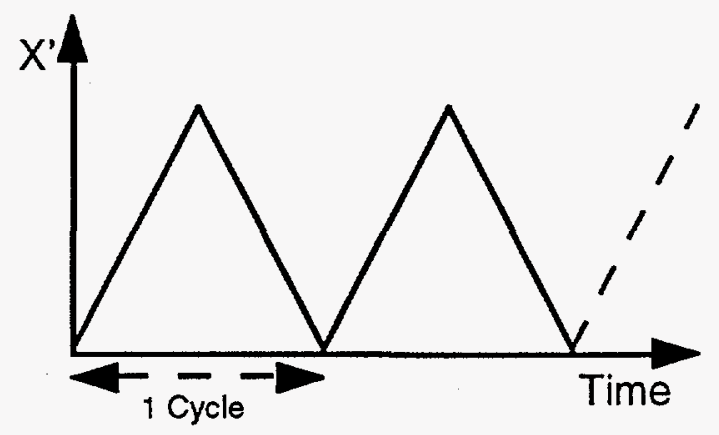

Figure 6. A schematic diagram for the position of the target relative to a moving thermal spray gun as a function of time. 
specific value for $\mathrm{X}^{\prime}$ from Figure 6, which in turn gives specific values for $\mathrm{w}, \mathrm{D}$ and $\theta$, from Figure 5. These time dependent values for $\mathrm{w}, \mathrm{D}$ and $\theta$ are applied uniformly over the length of the target included in the analysis. The spatial variations of $\mathrm{w}, \mathrm{D}$ and $\theta$ over the length of the target are neglected. That is, the length of target included in the analysis is typically $8 \mathrm{~mm}$. A strict application of the data shown in Figure 5 for the case when the position of the model target straddles two zones, e.g. zones 3 and 4 , would require the use of zone 3 values for $w, D$ and $\theta$ for one portion of the target, while zone 4 values would be used for the remaining portion of the target. This is not done, rather the model target is exposed to only one spray zone at any one time.

\section{III.2.2 Describing the Mass Flux in Terms of Droplets}

The thermal spray is assumed to consist of a large number of droplets that are randomly distributed within the spray. To describe the thermal spray mass flux in terms of the droplets, the droplet mass flux field is divided into a large number of small bins, as shown in Figure 7.

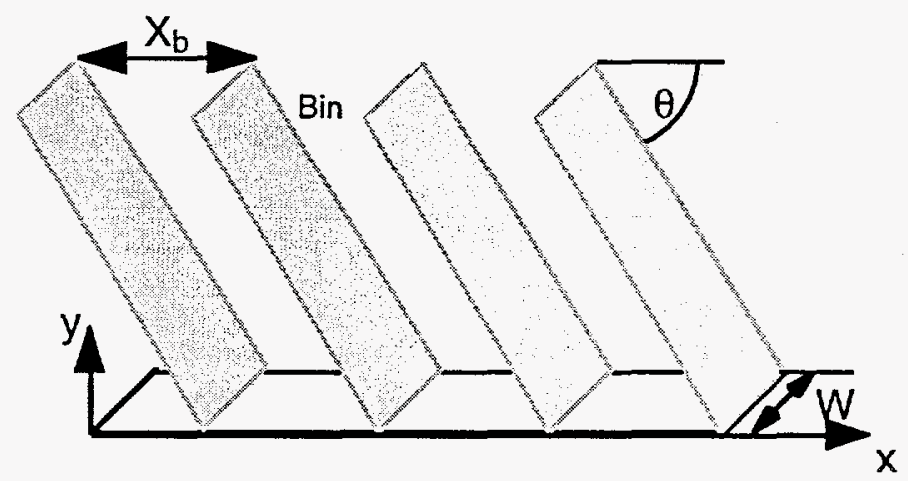

Figure 7. A schematic diagram showing how the droplet mass flux field is divided into a large number of small bins.

The bins are aligned with the direction of the droplet trajectories, $\theta$. The mass flux, $\mathrm{m}$, incident on the target surface may be expressed in terms of the deposition of finite sized droplets as:

$$
\mathrm{m}=\frac{\mathrm{V}_{\mathrm{D}} \rho}{\mathrm{A}_{\mathrm{b}} \delta \mathrm{t}_{\mathrm{a}}}
$$

where $V_{D}\left(=\pi D^{3 / 6}\right)$ is the volume of a spherical droplet, $A_{b}$ is the cross sectional area of a bin measured normal to the direction of the mass flux vector, and $\delta t_{a}$ is the 'average' time interval between droplet impacts on the portion of the target surface within a bin. In order to simulate the stochastic nature of the thermal spray, where the droplets in the spray impact the target with variable intervening time intervals, the actual time intervals between droplet impacts within a bin are given random values within $\pm 50 \%$ of some mean value, $\delta \mathrm{t}_{\mathrm{m}}$. This is done with a random number generator and computing a particular value of the time interval, $\delta \mathrm{t}$, as:

$$
\delta \mathrm{t}=\delta \mathrm{t}_{\mathrm{m}}\left(0.5+R_{\mathrm{n}}\right)
$$


$R_{n}$ is a random number between 0 and 1 with a uniform probability of a value occurring anywhere in that range. In order to obtain the relationship between $\delta t_{m}$ and $\delta t_{a}, \delta t$ from Equation (4) is substituted for $\delta \mathrm{t}_{\mathrm{a}}$ in Equation (3), which is then integrated over a large number of droplet impacts to obtain a good statistical sampling of all the potential values of $\delta$. Since $R_{n}$ has a uniform probability of occurring anywhere within the interval between 0 and 1, integrating Equation (3) over a large number of impacts, $N$, is equivalent to integrating Equation (3) with respect to $R_{\mathbf{n}}$ (where $\delta$ from Equation (4) has been substituted for $\delta t_{a}$ in Equation (3)), over the interval $0<R_{n}<1$. This gives the relationship in Equation (5),

$$
\delta t_{m}=\delta t_{a} \ln 3 .
$$

$\delta \mathrm{t}_{\mathrm{a}}$ is not equal to $\delta \mathrm{t}_{\mathrm{m}}$, because $\delta \mathrm{t}$ appears in the denominator of Equation (3), so $\delta \mathrm{t}_{\mathrm{a}}$ is not the true average of $\delta \mathrm{t}$, rather $1 / \delta \mathrm{t}_{\mathrm{a}}$ is the average value of $1 / \delta \mathrm{t}$. The desired result is the relationship between $\delta t_{m}$ to be used in Equation (4) for generating the time intervals between droplet impacts within a bin, and the specified values of $\mathrm{m}$ and $\mathrm{D}$, and is given in Equation (6):

$$
\delta t_{m}=\frac{\ln (3) V_{D}}{A_{b} m}
$$

The cross sectional area of a bin is evaluated as, $A_{b}=X_{b} W \sin \theta ; X_{b}$ is the length of a bin measured in the $\mathrm{x}$-direction, $\mathrm{W}$ is the width of the model target (and bin, see Figure 7) and may be chosen to be a unit width, but as discussed below, it is chosen to equal the diameter, $d$, of a typical splat.

A special case for the mass flux is the continuous distribution which deposits mass on all exposed points on the coating surface simultaneously. This is different from the stochastic deposition of finite sized droplets where a droplet may impact one exposed point on the coating surface while impacts on other exposed points may not occur until some time later. The amount of mass deposited by the continuous mass flux distribution on each exposed point on the coating surface, at each time step, is given by, $\mathbf{m} \bullet \mathbf{n} \cdot|\Delta \mathbf{r}| \cdot \mathbf{W} \cdot \Delta t$, where $|\Delta \mathbf{r}|$ is the node point spacing. Each element of mass deposited is assumed to spread out over a distance, $L$, given by some specified value for the product, $\xi \mathrm{D}$.

\section{III.2.3 Shading}

When large roughness elements, called scallops, develop on the coating surface, portions of the coating surface downstream of the scallops can be shaded from the thermal spray mass flux. This can have large effects on the subsequent development of the shape of the coating surface. Shaded regions on the coating surface are detected in the analysis by defining the thermal spray mass flux in terms of streamlines. Streamlines define the paths that thermal spray droplets follow to reach the surface of the coating. Shading occurs at a point on the surface when the streamline which intersects that point also intersects another point (or points) on the coating surface which is further upstream (see Figure 8).

It is assumed that close to the coating surface (e.g. within a few hundred microns) the streamlines can be approximated as straight lines. Then, Equation (7) defines a streamline where $\theta$ is the angle of inclination of the streamline with respect to the target (horizontal), and $\mathrm{x}_{S}$ is a constant defining one particular streamline. 


$$
y=\frac{\left(x-x_{s}\right)}{\tan \theta}
$$

The streamlines which intersect each point with coordinates $(x, y)$ on the coating surface, are defined by their respective value of $x_{S}=x-(y / \tan \theta)$. Consider a specific streamline intersecting the coating surface shown in Figure 8. Since the direction of a thermal spray droplet following the streamline is toward the coating surface, i.e. from larger values of $y$ toward smaller values of $y$, the intersection point with the largest value of $y$ is the (true) impact point for droplets following this streamline and the remaining intersection points represent shaded points.

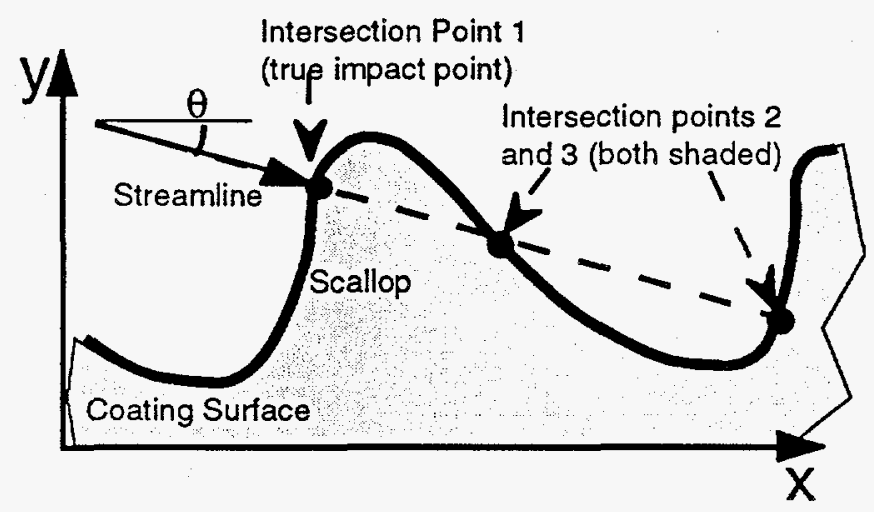

Figure 8. A schematic of the use of streamlines to determine the point on the coating surface which is exposed to the thermal spray mass flux.

The streamlines which intersect the coating surface are defined by computing their respective values of $\mathrm{x}_{\mathrm{S}}$. Streamlines which intersect more than one point on the coating surface are noted along with the points that they intersect. The intersection point with the largest value of $y$ is identified as the impact point for each streamline, and the remaining points are identified as shaded. This defines a set of unshaded, or potential impact points on the coating surface.

\section{III.2.4 Droplet Spreading}

When a thermal spray droplet impacts on the target surface it spreads out into a splat, which, for an impact that is perpendicular to a smooth surface, yields the shape of a flat circular disk. Perpendicular impacts on a rough surface often have 'star like' shapes [Smith, Neiser and Dykhuizen (1994)]. See Dykhuizen (1994) for a review of the impact phenomena of thermal spray droplets. Analyses for the size of a splat as a function of the Reynolds number of a thermal spray droplet have yielded relationships [Madejski 1976, Fukanuma 1994] of the form of Equation (8),

$$
\xi \equiv \frac{L}{D}=c e^{e}
$$

where $\xi$ is a spread coefficient defined as the ratio of the diameter of the disk shaped splat, $L$, to the diameter of the impacting droplet, $\mathrm{D}$. Re is the droplet Reynolds number, $\operatorname{Re}=u D \rho / \mu$, where $u$ is the impact velocity and $\rho$ and $\mu$ are the density and viscosity of the molten metal. These analyses show that the primary mechanism which limits the spreading of a droplet upon impact is the 
conversion of the kinetic energy in the droplet into thermal energy by the viscous dissipation which occurs during the distortion and spreading of the droplet into a disk. Madejski (1976) obtained values for the coefficient, c, and exponent, e, in Equation (8) of 1.2941 and 0.2 respectively. Fukanuma (1994) obtained values for $\mathrm{c}$ and $\mathrm{e}$ of 1.06 and 1/6, respectively. For typical values for the velocity and size of steel thermal spray droplets of $u=230 \mathrm{~m} / \mathrm{s}$ and $\mathrm{D}=30 \bullet 10^{-} 6 \mathrm{~m}$, the values of $\xi$ obtained from the results of Madejski and Fukanuma are 8 and 5, respectively.

Experimental measurements of $\xi$ for thermal spray droplets have been made and show much experimental scatter. Madejski (1976) measured $5<\xi<6$ for the impact of plasma sprayed alumina droplets on (presumably) smooth surfaces. Fantassi, Vardelle, Vardelle and Fauchais (1993) measured $1<\xi<6$ and Bianchi, Blein, Lucchese, Vardelle, Vardelle and Fauchais (1994) measured $\xi=5$ for the impact of plasma sprayed zirconia droplets on smooth surfaces. Moreau, Gougeon and Lamontagne (1995) studied the effect of target surface roughness on $\xi$ for plasma sprayed molybdenum droplets. They measured $7<\xi<10$ for smooth targets, $5<\xi<7$ for fine grit blasted targets ( $\mathrm{R}_{\mathrm{a}}=1$ micron), and $4<\xi<5$ for coarse grit blasted targets ( $\mathrm{R}_{\mathrm{a}}=7$ microns).

Impacts that are not perpendicular have also been studied experimentally by Hasui (1970), Madejski (1976) and Montavon, Coddet, Sampath, Herman and Berndt (1994). In general, nonperpendicular impacts result in an elongation of the splat in the direction of the component of the droplet velocity parallel to the target, such that the splat has more of an oval shape. The data by Madejski (1976) for the length of the splat, L, (which is different from the width of a splat for nonperpendicular impacts) is presented in terms of a modified spread coefficient defined as $\xi^{\prime}=L / D$. Figure 9 shows $\xi^{\prime} / \xi$ as a function of the impact angle, where $\xi^{\prime}=\xi$ for a perpendicular impact. A quadratic polynomial function of the impact angle, $\beta\left(\beta=90^{\circ}\right.$ is perpendicular), given in Equation (9), provides a good approximation to the data as shown in Figure 9.

$$
\frac{\xi^{\prime}}{\xi}=3.5-0.0556 \beta+0.000309 \beta^{2}
$$

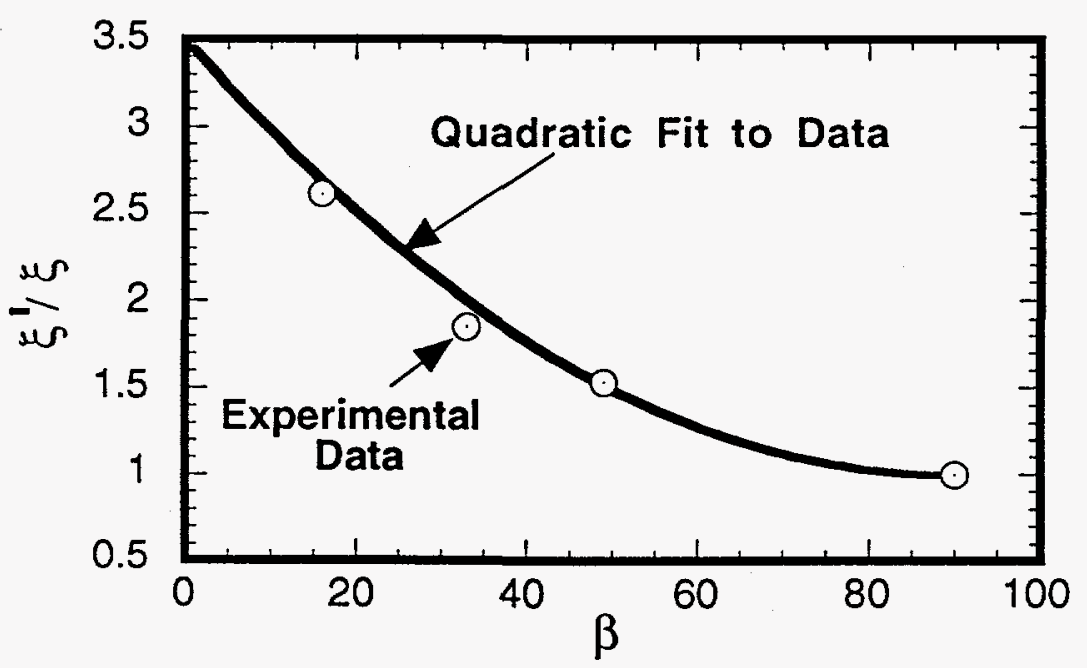

Figure 9. The data by Madejski (1976) for the splat length as a function of impact angle. 
It is assumed that when a droplet impacts on the target surface, it spreads out to form a splat with a length, L, a width, W, and a uniform thickness, T. Due to the non-perpendicular impact of the thermal spray jet in the thermal spray process studied here, there is a predominance of nonperpendicular droplet impacts. Hence, the splat length is computed using Equation (9) to account for the elongation effect that non-perpendicular impacts have on the shape of the splats. A prescribed value of $\xi$ (typically a value of 3 for rough surfaces) corresponding to a perpendicular impact is used along with Equation (9) for $\xi$ to obtain the length of a splat in the model. Here, the impact angle, $\beta$, is the local impact angle which depends not only on the spray angle, $\theta$, but also on the local angle of the coating surface, as shown in Figure 10. The length of a splat is then given by Equation (10).

$$
L=\xi^{\prime} \mathrm{D}
$$

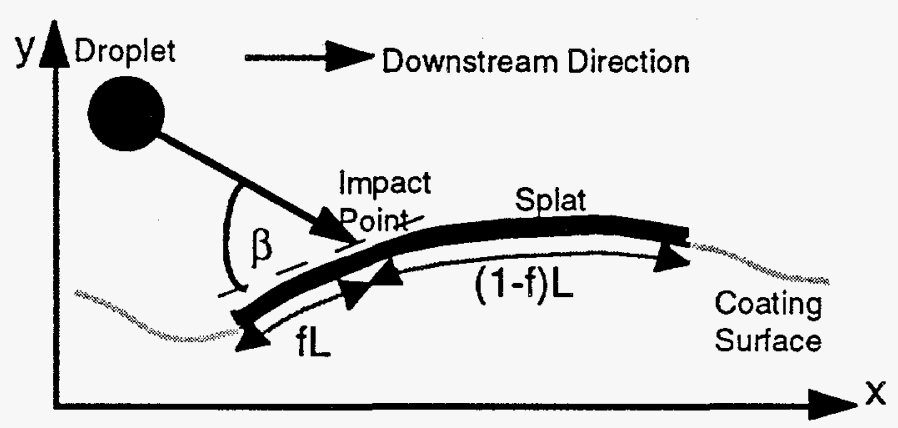

Figure 10. A schematic diagram showing the location of a splat relative to the droplet impact point on the coating surface.

It is assumed that the position of the splat relative to the droplet impact point depends on the impact angle, $\beta$. The appearance of the splats obtained by Madejski (1976) created by nonperpendicular impacts indicates that the splat length is not centered about the impact point, rather the position of the splat center is downstream of the impact point, i.e. in the direction of the component of the droplet velocity locally parallel to the target surface. This is consistent with the analysis of Taylor (1961) for a two-dimensional fluid jet impinging on a flat surface at a nonperpendicular angle. From the conservation of momentum, some of the mass in the jet will flow upstream from the impact point (in the opposite direction of the component of the jet velocity parallel to the target surface), and the remaining mass in the jet will flow downstream. The fraction of the jet that flows upstream, $f$, is given by, $\sin ^{2}(\beta / 2)$, where $\beta$ is the jet spray angle defined in Figure 10 for a droplet impact. Although a droplet impact is not the same as a jet impact, it is assumed that similar phenomena occur where most of the mass in the droplet flows toward the downstream direction of the impact point. Due to the absence of data for the relationship between $f$ and $\beta$ for a droplet impact, a simple linear relationship is used here for the fraction of the splat located upstream of the impact point, $f$, as given in Equation (11).

$$
f=\frac{\beta}{180}
$$

Using Equation (11) for $\mathrm{f}$ positions the center of the splat closer to the impact point compared to that which would be obtained if the relationship of $\sin ^{2}(\beta / 2)$ were used for $f$. Equation (11) gives 
the result, $f=1 / 2$, for $\beta=90$, i.e. the splat center is located at the impact point for a perpendicular impact, as it should based on symmetry considerations.

The splat is assumed to have a uniform thickness. This appears to be a reasonable assumption, at least for normal droplet impacts, based on the measurements by Bianchi et. al (1994). The splat thickness, $T$, is computed based on the volume of a droplet, $V_{D}$, and the length of the splat, L, as shown in Equation (12). Note that the splat shape is assumed to be rectangular in this two-dimensional model.

$$
\mathrm{T}=\frac{\mathrm{V}_{\mathrm{D}}}{\mathrm{LW}}
$$

The splat width, $\mathrm{W}$, is assumed to equal the splat size for a normal impact, $\mathrm{W}=\xi \mathrm{D}$. There is more than one droplet size included in the thermal spray mass flux distributions described in section III.2.1, so a direct application of this equation for W would lead to different splat widths. Since the model is two dimensional it is desirable to give all splats the same width. This is done by basing W on the size used for the main spray droplets, $\mathrm{D}_{\mathrm{m}}$, as shown in Equation (13), for all splats, regardless of the size of the droplets forming the splats.

$$
\mathrm{W}=\xi \mathrm{D}_{\mathrm{m}}
$$

This value of $\mathrm{W}$ is also used for the width of a mass flux bin described in section III.2.2. This is consistent since the rate at which mass is deposited is affected by the time intervals between droplet impacts, which in turn is affected by the width of a bin as shown in Equation (6), where $\mathrm{A}_{\mathrm{b}}=\mathrm{X}_{\mathrm{b}} \mathrm{W} \sin \theta$. At the same time, the rate at which the thickness of the coating increases depends on the width of the splats, as shown in Equation (12). It is consistent to base the rate at which mass is deposited, and the rate at which the coating thickness increases, on the same width, W. Also, it is assumed that the splat follows the local contour of the underlying target surface.

\section{III.2.5 Initial Condition}

The initial condition for the shape of the target surface is the shape of the pre-roughened substrate profile. A simple sine wave can be used to approximate this pre-roughened surface. A more realistic shape is obtained by using profilometer data for the shape of an actual pre-roughened surface. This is obtained from a laser profilometer which is used to scan the surface of a preroughened substrate. The shape of the surface is described in terms of the coordinates of a large number of equally spaced points.

\section{III.2.6 Boundary Condition}

Recall that only a portion of the actual coating is included in the simulations. This computed portion can be affected by portions of the coating surface not included in the model by the shading which they cause, i.e. the portions of the surface not included in the model may cast shadows (and prevent the direct deposition of the thermal spray mass flux) on the computed portion of the coating surface. The computed portion of the coating surface can also be affected by droplet impacts which occur outside of the computed portion of the surface but spread out into splats such that mass is deposited on the computed portion of the surface. These effects are accounted for by using a periodic boundary condition where it is assumed that the computed surface repeats itself. Thus, a shadow cast by one end of the computed surface is assumed to fall on the other end. Similarly, the portion of a splat which moves off one end of the computed surface is assumed to move onto the other end of the computed surface. 


\section{Solution Methodology}

A numerical procedure is used to implement the analysis described above. The surface of the coating is defined with a set of equally spaced node points. These node points track the position and shape of the coating surface as they change due to the deposition of the sprayed material. Equation (1) is discretized as shown in Equation (14).

$$
\frac{\Delta \mathbf{r}_{k}}{\Delta t}=g_{k} \mathbf{n}_{k} \Rightarrow \frac{\Delta x_{k}}{\Delta t} \mathbf{i}+\frac{\Delta y_{k}}{\Delta t} \mathbf{j}=g_{k}\left(\chi_{k} \mathbf{i}+\gamma_{k} \mathbf{j}\right)
$$

where $\mathbf{i}$ and $\mathbf{j}$ are the unit vectors in the $\mathbf{x}$ and $\mathrm{y}$ directions, respectively, and $\chi_{\mathrm{k}}$ and $\gamma_{\mathrm{k}}$ are the $\mathrm{x}$ and $y$ components, respectively, for the unit vector locally normal to the coating surface, and the subscript, $\mathrm{k}$, refers to a specific node. This equation can be split into its $\mathbf{i}$ and $\mathbf{j}$ components to obtain equations for the change in the $\mathrm{x}$ and $\mathrm{y}$ coordinates, $\Delta \mathrm{x}_{\mathrm{k}}$ and $\Delta \mathrm{y}_{\mathrm{k}}$, respectively, which take place due to the mass deposition during the time step, $\Delta t$, for the node $k$. The components of the local normal unit vector at node $\mathrm{k}$ are computed using Equations (15), where $\mathrm{k}-1$ and $\mathrm{k}+1$ refer to the neighboring nodes as shown in Figure 11.

$$
\chi_{k}=\frac{y_{k-1}-y_{k+1}}{\left|\mathbf{r}_{k+1}-\mathbf{r}_{k-1}\right|}, \quad \gamma_{k}=\frac{x_{k+1}-x_{k-1}}{\left|\mathbf{r}_{k+1}-\mathbf{r}_{k-1}\right|}
$$

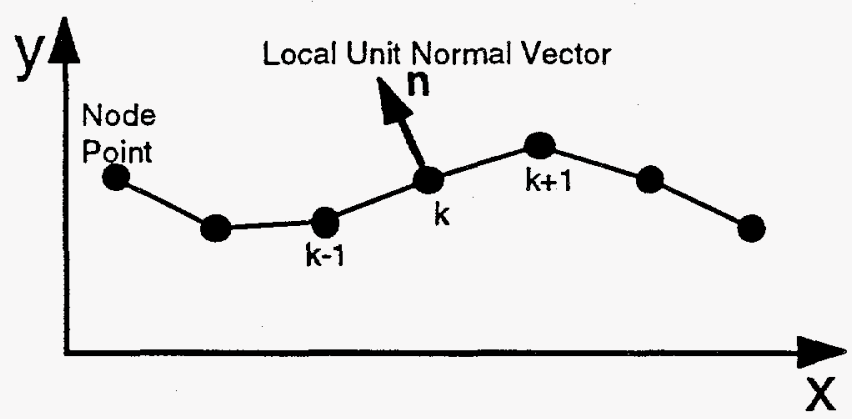

Figure 11. A schematic of the node point distribution along the coating surface.

Equation (14) is solved for each node using an explicit first order method where current values of the nodal coordinates are used to determine the values for $g_{k}$ and for $\chi_{k}$ and $\gamma_{k}$. At the beginning of each time step the nodes are equally spaced along the coating surface and the mass flux characteristics $m, \theta$ and $D$, are updated for all nodes, $k$, in preparation for computing values for $g_{k}$ for the current time step. The extent of shading is determined to identify the set of nodes exposed to the current mass flux. All droplet impact events are then identified along with their position. A droplet impact event occurs within a bin if $t<t_{b}+\delta t_{b}<t+\Delta t$, where $t_{b}$ is the time of the last droplet impact in bin $b$, and $\delta t_{b}$ is the next time interval between droplet impacts within bin $b$. Thus, the time for the next droplet impact in bin $b$ is $t_{b}+\delta t_{b}$, and if this time occurs between the current time, $t$, and the time at the next time step, $t+\Delta t$, then a droplet impact occurs in bin $b$. The location of the impact is the exposed node closest to the center of bin $b$. 
After all droplet impact events are identified, droplet spreading is computed. For each droplet impact event, the local impact angle, $\beta$, is computed using Equation (16) which is based on the relationship between the dot product of two unit vectors and the angle between them, $\cos \beta=-$ $(\mathrm{m} / \mathrm{m}) \bullet \tau_{\mathrm{k}} \cdot(\mathrm{m} / \mathrm{m})$ is the unit vector parallel to the mass flux vector (given by $\left.\cos \theta \mathbf{i}+\sin \theta \mathbf{j}\right)$ and $\tau_{k}=-\chi_{k} \mathbf{i}+\gamma_{k} \mathbf{j}$, which is the unit vector locally tangent to the coating surface.

$$
\beta=\cos ^{-1}\left(\sin \theta \cdot \chi_{k}-\cos \theta \cdot \gamma_{k}\right)
$$

The length of the splat, L, is computed using Equations (9) and (10), the position of the splat relative to the impact point, $f$, is computed using Equations (11) and (16), and the thickness of the splat, $T$, is computed using Equations (12) and (13). The quantity, $T / \Delta t$, is then added to $\mathrm{g}_{\mathrm{k}}$ for all nodes, $\mathrm{k}$, which are within the distance $\mathrm{fL}$ upstream of the impact point, and to all nodes, $\mathrm{k}$, which are within the distance (1-f)L downstream of the impact point. This is done for each droplet impact event. This gives the values for $\mathrm{g}_{\mathrm{k}}$. The coordinates of the node points are then incremented using Equation (17), and time is incremented as $t^{n+1}=t^{n}+\Delta t$, where the superscripts, $n$ and $n+1$, are used to denote old and new values, respectively.

$$
x_{k}^{n+1}=x_{k}^{n}+g_{k} \cdot \chi_{k} \cdot \Delta t, \quad y_{k}^{n+1}=y_{k}^{n}+g_{k} \cdot \gamma_{k} \cdot \Delta t
$$

In general, the process of incrementing the nodal coordinates can result in the generation of loops in the simulated coating surface, as shown in Figure 12. This can occur when a crevasse in

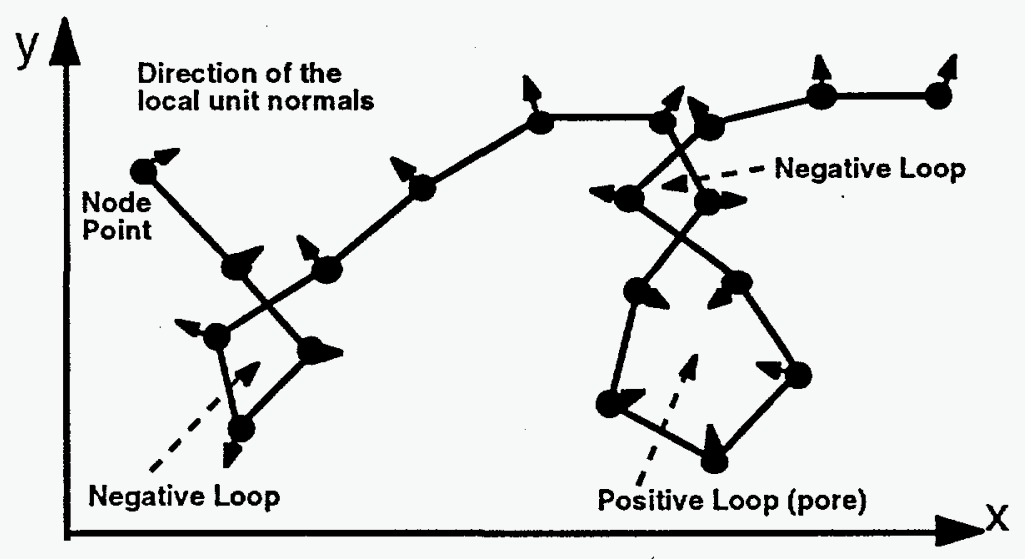

Figure 12. A schematic of erroneous loops in the computed coating surface.

the surface forms where one part of the surface is close to, and faces another part of the surface. Due to mass deposition, both parts of the surface advance in their respective forward directions, i.e. the direction in which they advance due to mass deposition, which may result in a cross over. Both 'negative' and 'positive' loops may form. A 'negative' loop is one where the forward facing direction of the nodes, indicated by the direction of the local unit normal vectors shown in Figure 12 , is pointed outward away from the center of the loop, and a 'positive' loop is one where the forward facing direction is pointed inward towards the center of the loop. A 'positive' loop represents a void or pore which is entrapped within the coating. It is important to eliminate both types of loops because they can result in the generation of erroneous results. During the course of a simulation the surface is searched periodically for 'negative' and 'positive' loops. The 'negative' loops are eliminated by redistributing the nodes along the path of that portion of the coating surface which does not include the loop, as shown in Figure 13. 'Positive' loops are also eliminated, but 


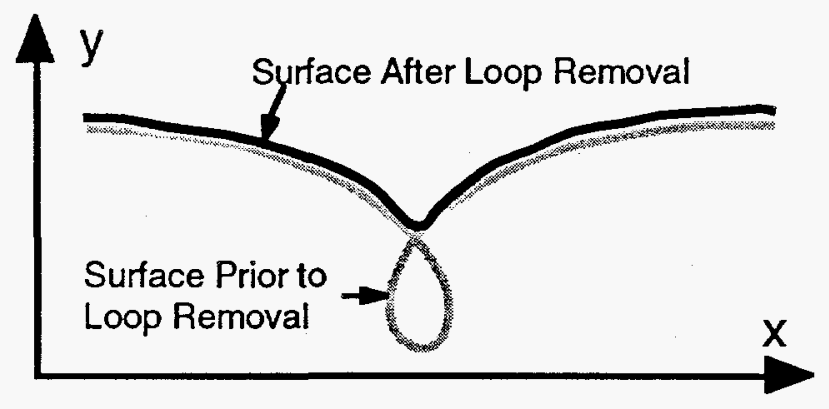

Figure 13. A schematic diagram of the computed coating surface after removal of a loop.

first their size, shape and position are recorded to indicate that a pore has formed. The process of eliminating loops is not necessarily done every time step, e.g. it may be done every five time steps. It is important, however, to search the surface for loops often so that they may be eliminated before they become large.

In general, the process of incrementing the nodal coordinates also results in a non-equal distribution of the node points along the coating surface. If the non-uniformity in the node spacing becomes large, this also can result in the generation of erroneous results. Hence, the nodes are redistributed along the coating surface after every time step in order to maintain the uniform node spacing.

This completes one time step, and at this point the calculations begin again for the next time step beginning with an update of the mass flux characteristics for the current time and an evaluation of the shading that occurs on the current shape of the coating surface. 


\section{RESULTS}

The results for the coating roughness are presented in terms of the roughness variables, $R_{a}$ and $S_{m}$, which provide measures for the average height and spacing, respectively, of the scallops (i.e. roughness elements) which form on the surface of the coating. $\mathrm{R}_{\mathrm{a}}$ is defined as:

$$
R_{a}=\frac{1}{\Delta X_{t}} \int_{0}^{\Delta x_{t}}\left|y-y_{m}\right| d x
$$

where $\Delta X_{t}$ is the length of the model target, and $y_{m}$ is the average height of the coating surface, also called the coating thickness. $S_{m}$ is the average distance between the peaks of the scallops on the coating surface as measured parallel to the $\mathrm{x}$-axis, and is given by Equation (19). As shown in

$$
\mathrm{S}_{\mathrm{m}}=\frac{\Delta \mathrm{X}_{\mathrm{t}}}{\text { Number of Peaks }}
$$

Figure 14, the peak of a scallop is defined as the highest point on the coating surface with a height, $\mathrm{y}$, that is above the elevation given by $y_{\mathrm{m}}+0.5 * \mathrm{R}_{\mathrm{a}}$, and is separated from other peaks by points on the coating surface with heights that are below the elevation given by $y_{m}-0.5 * R_{a}$, called valleys. Note that this is the definition used here, and that other definitions for $S_{m}$ are possible [Sander, 1989].

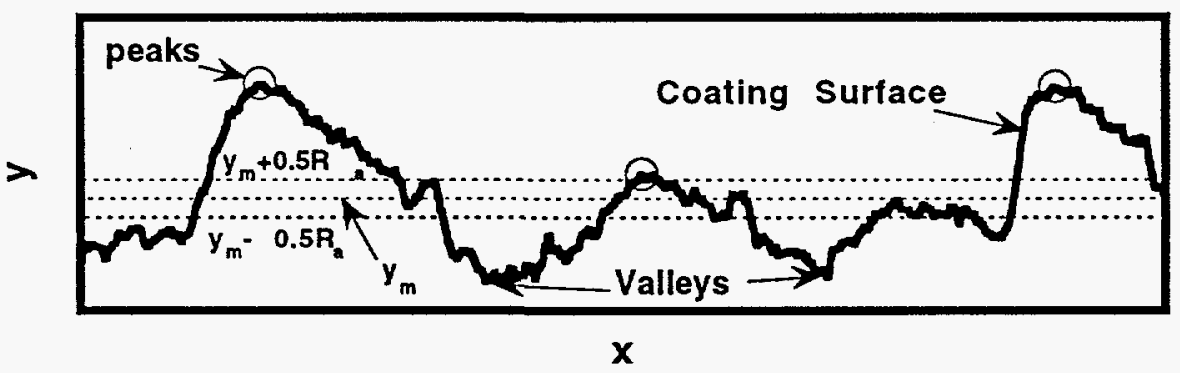

Figure 14. A schematic diagram for the coating surface and the arrangement of peaks and valleys.

$\mathrm{R}_{\mathrm{a}}$ and $\mathrm{S}_{\mathrm{m}}$ are used here to describe the coating surface. Note, however, that these variables are only average quantities and do not provide information about the deviations of the individual heights and spacing of the scallops from the average values.

Results are first presented for a set of simplified conditions where the mass flux was assumed to be continuous, a single value is used for the angle of the mass flux, $\theta$, and a simple sine wave was used to approximate the pre-roughness of the substrate. Then, results showing the effects of a mass flux composed of droplets which are deposited stochastically on the rough surface are presented. Next, results are presented for the mass flux distributions calculated by Lopez et. al (1995). Finally, results are presented for the mass flux distribution shown in Figure 5. 


\section{V.1. Continuous Mass Deposition on Simple Surfaces}

The results presented in this section were obtained from calculations using the continuous mass distribution. Each element of mass deposited is assumed to spread out over a distance, $\mathrm{L}$, given by some specified value for the product, $\xi \mathrm{D}$, where the spread coefficient, $\xi$, is used which is independent of the local impact angle, $\beta$. In addition, a single value is used for the angle of the mass flux, $\theta$, and a sine wave with a wavelength, $S_{m 0}$, and a roughness of, $R_{a 0}$, is used to approximate the pre-roughness.

For the special case of $\mathrm{R}_{\mathrm{a} 0}=0$, i.e. a smooth initial substrate, the coating surface is also smooth for a continuous mass distribution, independent of $\theta$ and $L$. While this is a desirable coating surface, the coating does not bond well to a smooth substrate, so pre-roughened substrates must be used. For $R_{a 0} \neq 0$, the results for the shape of the coating surface were found to depend on the spray conditions, $\theta$ and $\mathrm{L}$, and on the pre-roughness characteristics, $S_{\mathrm{m} 0}$ and $\mathrm{R}_{\mathrm{a} 0}$. Figure 15 shows typical results for the shape of the coating surface where the product of two sine waves was used for the pre-roughened substrate. One sine wave had a wavelength of 100 microns and the

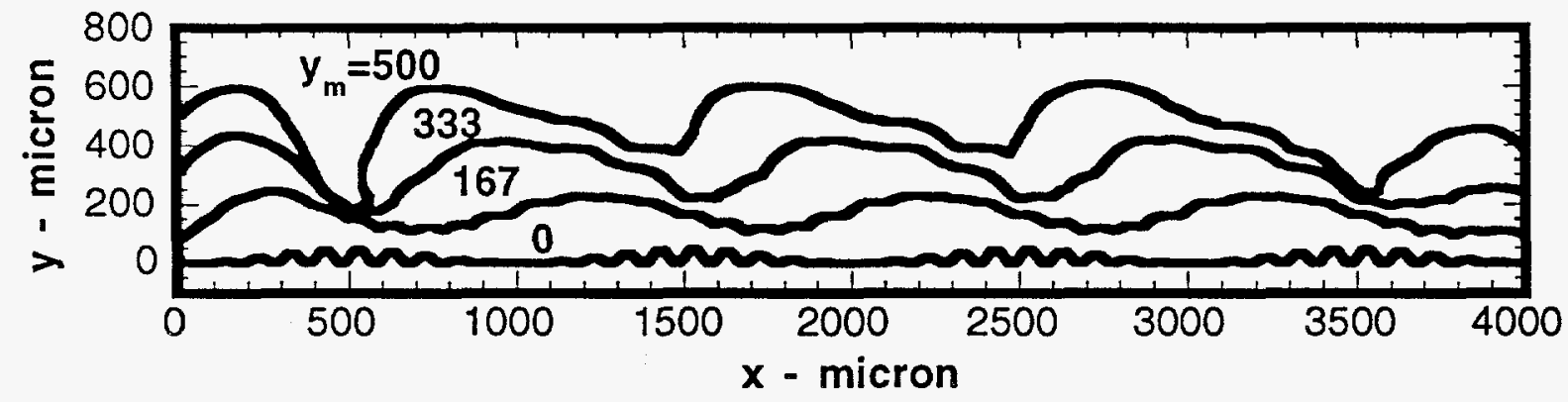

Figure 15. The shape of the coating surface for a continuous mass flux distribution on a sine wave substrate for $\mathrm{L}=400$ microns, $\theta=26^{\circ}$ and $S_{m 0}=1000$ microns (Stable Scallop Regime).

other had a wavelength of 1000 microns. The smaller wavelength had only a small effect on the results, while the larger wavelength had a large effect and is the better measure for $S_{\mathrm{m} 0}$. These results are for a splat size, $\mathrm{L}$, of 400 microns, and a spray angle, $\theta$, of $26^{\circ}$, and were obtained from calculations which did not use the periodic boundary condition. (The results presented throughout most of this section were obtained during the early part of this study when the periodic boundary condition had not yet been implemented.) The results show that, starting from a preroughened surface with small roughness, large scallops can grow resulting in a coating surface with large roughness.

The results shown in Figure 15 have a simple structure, where $S_{m}=S_{m 0}$, i.e. there is a oneto-one correspondence between scallops and waves in the substrate. This is not always the case, where in general, the relationship between $S_{\mathrm{m}}$ and $S_{\mathrm{m} 0}$ depends on the ratio of $S_{\mathrm{m} 0} / \mathrm{L}$, and the spray angle, $\theta$. Figure 16 shows this relationship, where there are four different regimes in $S_{\mathrm{m} 0} / \mathrm{L}$ versus $\theta$ space, called the 'stable scallops', 'no scallops', 'merging scallops' and 'bifurcating scallops' regimes. As the names imply, scallops grow in three of the four regimes. 
The 'stable scallop' regime is located at the middle of Figure 16 and is characterized by $\mathrm{S}_{\mathrm{m}}=\mathrm{S}_{\mathrm{m} 0}$. The result shown in Figure 15 is an example of the 'stable scallop' regime. Other results from this regime for $\mathrm{L}=400$ microns are shown in Figure $17 \mathrm{a}$ and $\mathrm{b}$, for $\theta=15^{\circ}$ and $\mathrm{S}_{\mathrm{m} 0}=700$ microns, and for $\theta=45^{\circ}$ and $S_{m 0}=1000$ microns, respectively. Although the results shown in Figures 15 and 17 are all from the 'stable scallop' regime, where $S_{m}=S_{m 0}$, there is a large difference in the value of $R_{a}$ for these results. Specifically, $R_{a}=330$ microns for $\theta=15^{\circ}, R_{a}=82$ microns for $\theta=26^{\circ}$, and $R_{a}=28$ microns for $\theta=45^{\circ}$. Thus, $R_{a}$ is strongly dependent on the spray angle, with $R_{a}$ decreasing with increasing $\theta$.

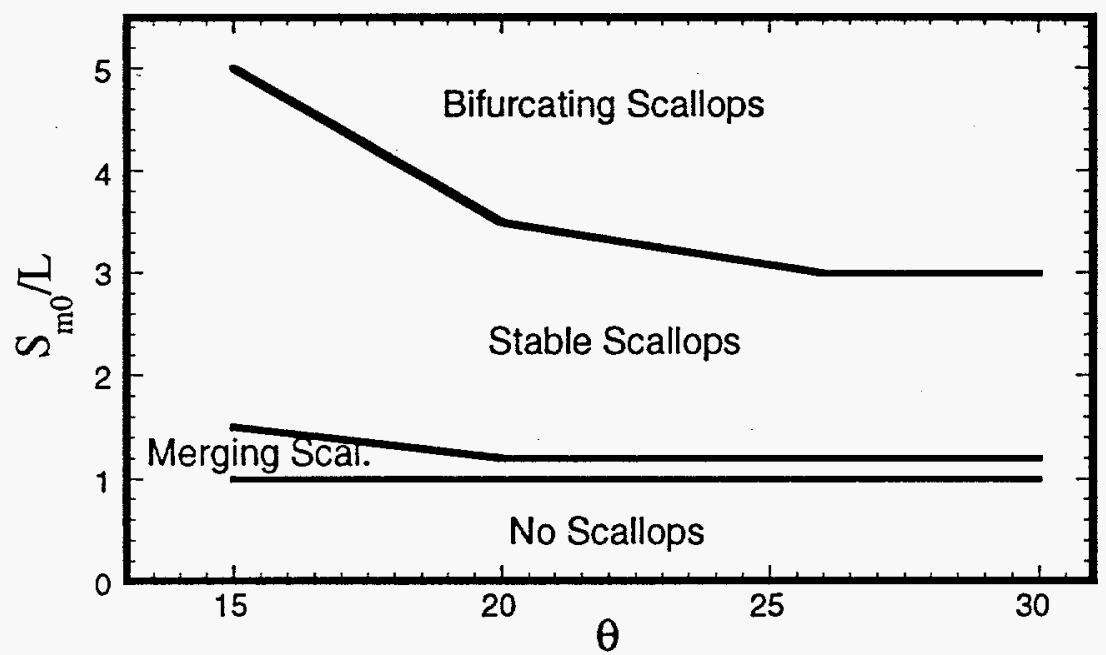

Figure 16. The four regimes for scallop growth in the input parameter space.

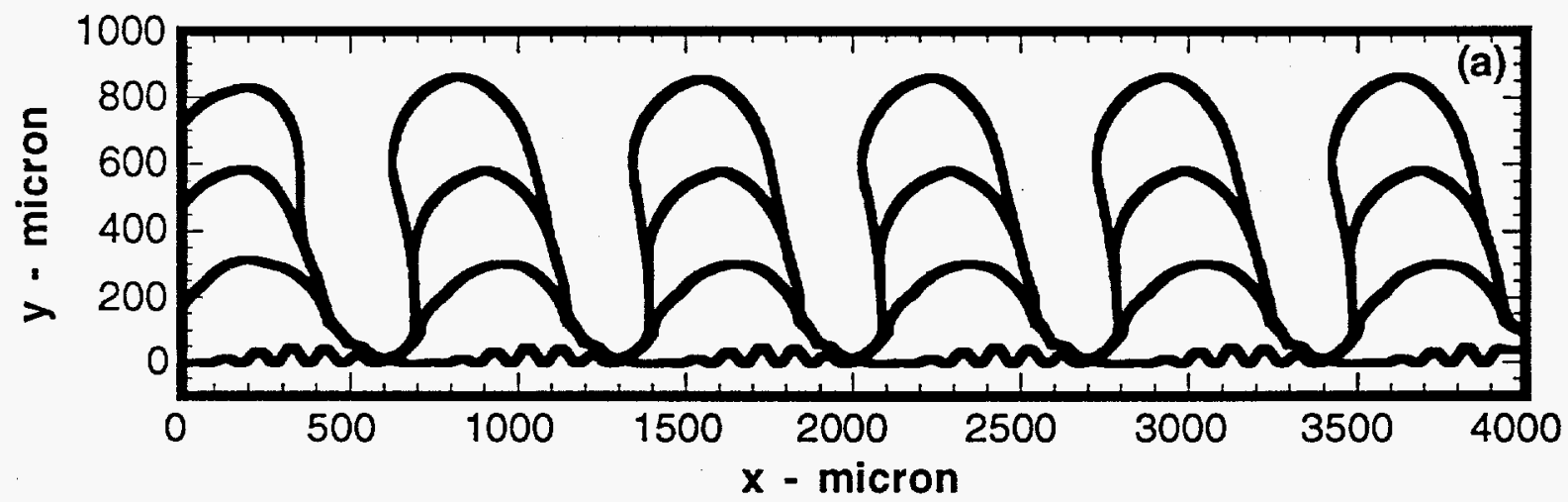

Figure 17a. The shape of the coating surface for a continuous mass flux distribution on a sine wave substrate for $\mathrm{L}=400$ microns, $\theta=15^{\circ}$ and $S_{m 0}=700$ microns (Stable Scallop Regime). 


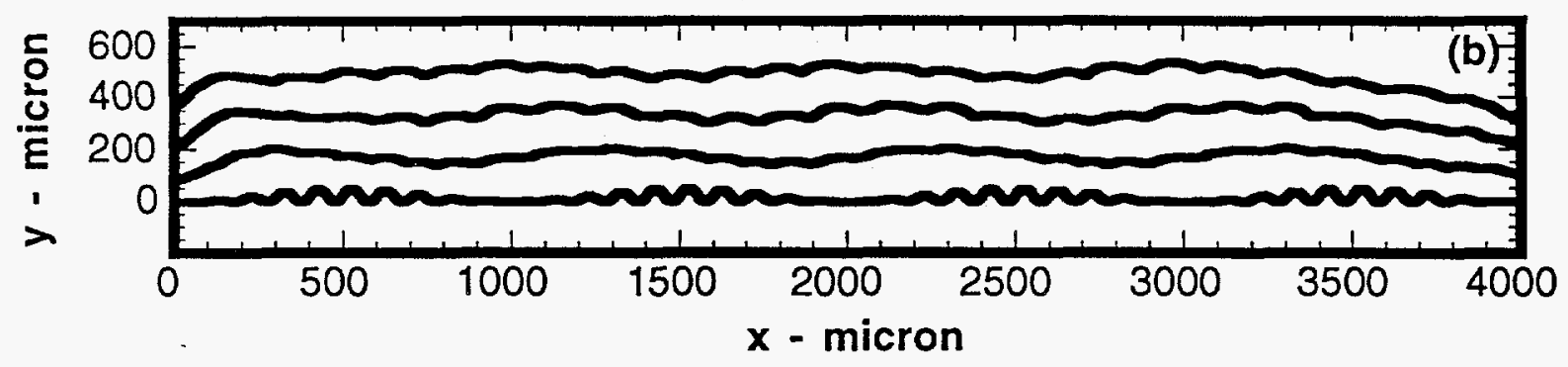

Figure 17b. The shape of the coating surface for a continuous mass flux distribution on a sine wave substrate for $\mathrm{L}=400$ microns, $\theta=45^{\circ}$ and $S_{m 0}=1000$ microns (Stable Scallop Regime).

In the 'bifurcating scallop' regime (cf. Figure 16) scallops begin to grow with $S_{m}=S_{m 0}$ early in the process, but then each scallop bifurcates into two scallops such that $S_{m}=S_{m 0} / 2$, as shown in Figure 18 for $\mathrm{S}_{\mathrm{m} 0} / \mathrm{L}=3.3$. Larger values of $\mathrm{S}_{\mathrm{m} 0} / \mathrm{L}$ can result in each scallop dividing into three (or presumably more) scallops. After bifurcation, the state of the coating surface resides in the stable scallop regime based on $\mathrm{S}_{\mathrm{m}} / \mathrm{L}=0.5 \mathrm{~S}_{\mathrm{m} 0} / \mathrm{L}=1.6$.

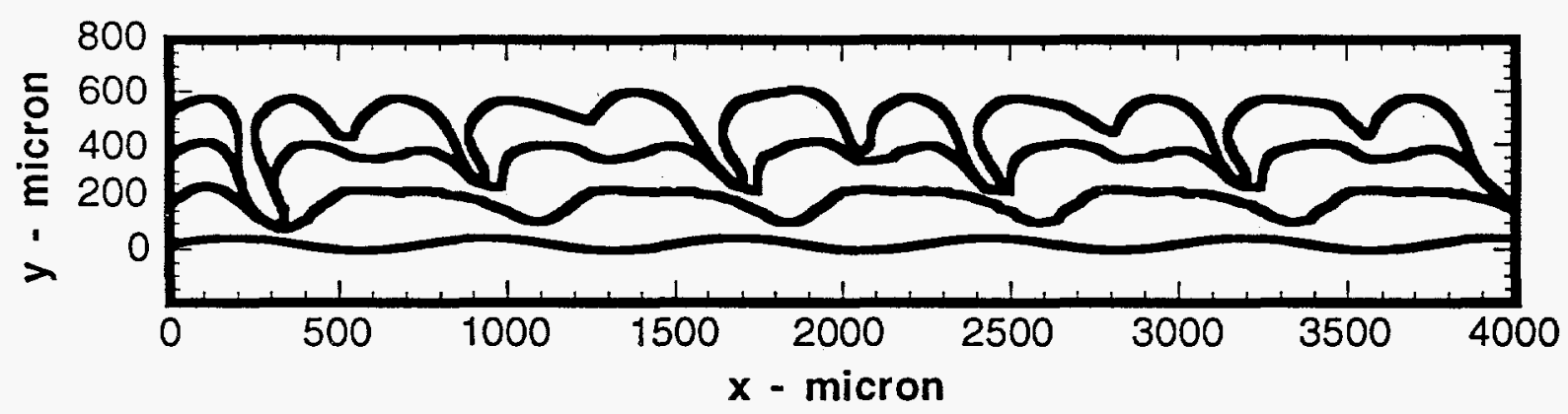

Figure 18. The shape of the coating surface for a continuous mass flux distribution on a sine wave substrate for $\mathrm{L}=230$ microns, $\theta=26^{\circ}$ and $\mathrm{S}_{\mathrm{m} 0}=750$ microns (Bifurcating Scallop Regime).

In the 'no scallop' regime of Figure 16, as the name implies, no scallops grow for $\mathrm{S}_{\mathrm{m} 0}<\mathrm{L}$. The results shown in Figure 19, for $\mathrm{S}_{\mathrm{m} 0} / \mathrm{L}=0.7$, is an example of a coating formed in this regime.

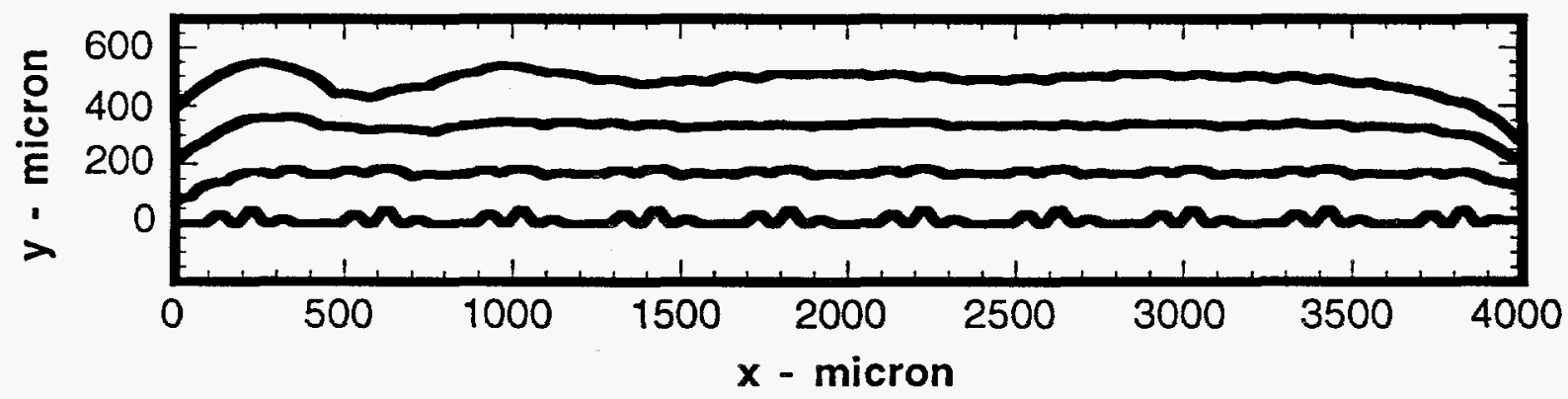

Figure 19. The shape of the coating surface for a continuous mass flux distribution on a sine wave substrate for $\mathrm{L}=550$ microns, $\theta=26^{\circ}$ and $\mathrm{S}_{\mathrm{m} 0}=400$ microns (No Scallop Regime). 
Here the pre-roughness on the substrate surface is quickly covered and a smooth surface prevails thereafter, except for the scallops which begin to form late in the process. These scallops are generated by the non-uniform shape near the edges of the calculated surface which result from the lack of a periodic boundary condition. These scallops would not appear if a periodic boundary condition were used. This is the same result that is obtained for a smooth substrate. Thus, in order for scallops to grow on the coating surface, they must be initiated. That is, a smooth coating surface is unstable and it remains smooth if there are no disturbances present in the substrate surface, such as a pre-roughness with a wavelength which is larger than the splat size.

In the 'merging scallop' regime scallops begin to grow with $S_{m}=S_{m}$ early in the process, but then each pair of scallops merge into one scallop such that $S_{m}=2 S_{m 0}$, as shown in Figure 20 for $S_{m 0} / L=1$. After merging, the state of the coating surface resides in the stable scallop regime based on $\mathrm{S}_{\mathrm{m}} / \mathrm{L}=2 \mathrm{~S}_{\mathrm{m} 0} / \mathrm{L}=2$.

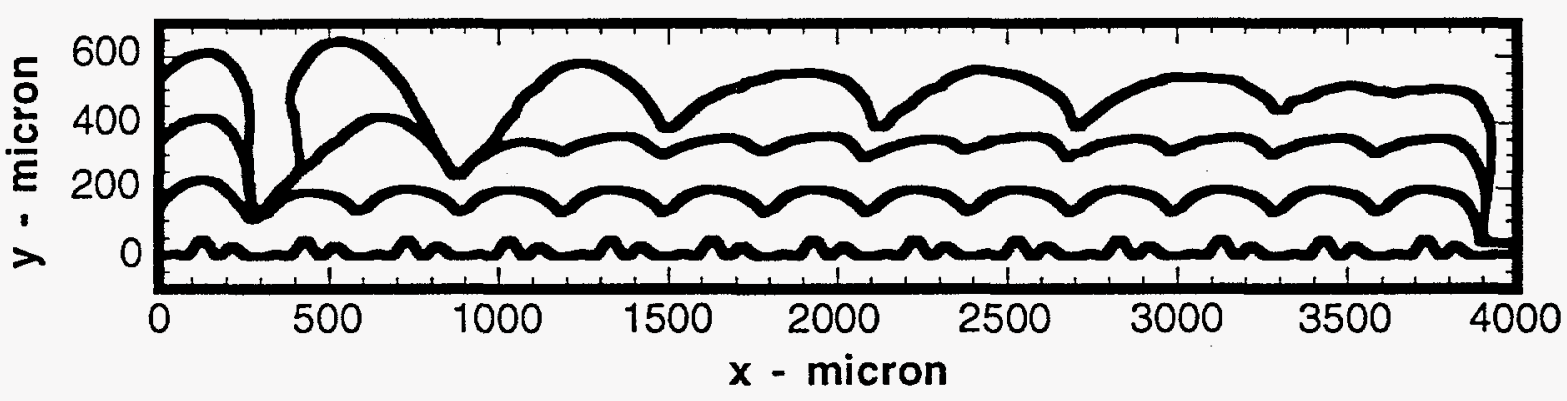

Figure 20. The shape of the coating surface for a continuous mass flux distribution on a sine wave substrate for $\mathrm{L}=300$ microns, $\theta=20^{\circ}$ and $S_{m 0}=300$ microns (Merging Scallop Regime).

The results presented above were for fairly simple and idealized conditions of a continuous mass flux, a single value for the spray angle and a pre-roughness characterized by a single wavelength, and show that large scallops can grow on the coating surface. For these conditions, the scallops grow stably with a spacing, $S_{m}$, such that $L<S_{m}<n L$, where $L$ is the splat size and $n$ is a factor which increases with decreasing spray angle, $\theta$. For $\theta=30^{\circ}, n=3$. For $S_{m} \neq S_{m 0}$, this stable value for $S_{m}$ results from the processes of bifurcation or merging. After the stable value for $\mathrm{S}_{\mathrm{m}}$ is established, it remains constant thereafter.

The development of a stable constant value for $S_{m}$ does not occur in real coatings. It will be shown later in this report that $S_{m}$ becomes larger than the stable value, due to scallop merging. This occurs due to non-uniformities in the shape of the coating surface, which can result from nonuniformities in the profile of the pre-roughened substrate, or it can result from the stochastic nature of the deposition of the finite sized droplets which make up the thermal spray. The results shown in Figure 21 illustrate the effect of a simple example for a target surface with a non-uniform shape. (These results were obtained from calculations using a periodic boundary condition). A continuous mass flux was used with $L=120$ microns and $\theta=26^{\circ}$. A sine wave with a wavelength, $S_{m 0}$, of 300 microns was used as the initial condition for the target profile shape. This gives $S_{m} / L=2.5$, which is well within the stable scallop regime where $S_{m}$ should remain constant and equal to $S_{m 0}$. The results shown in Figure 21a are for an initial condition where the heights of each wavelet in the pre-roughness profile are all the same. As expected, the scallops grow stably with $S_{m}=S_{m 0}$. The results shown in Figure $21 \mathrm{~b}$ are for an initial condition where the heights of the wavelets in the preroughness profile increase within each group of three, in a repeating pattern. Here the scallops, initially growing with $S_{m}=S_{m 0}$, merge with each group of three becoming one large scallop. The effect of the merging process is to increase the coating roughness from $R_{a}=92$ microns (Fig. 21a) 
to $\mathrm{R}_{\mathrm{a}}=133$ microns (Fig. $21 \mathrm{~b}$ ). This simple example illustrates the effect of scallop merging on $\mathrm{R}_{\mathrm{a}}$, although neither result shown in Figure 21 is a desirable coating profile

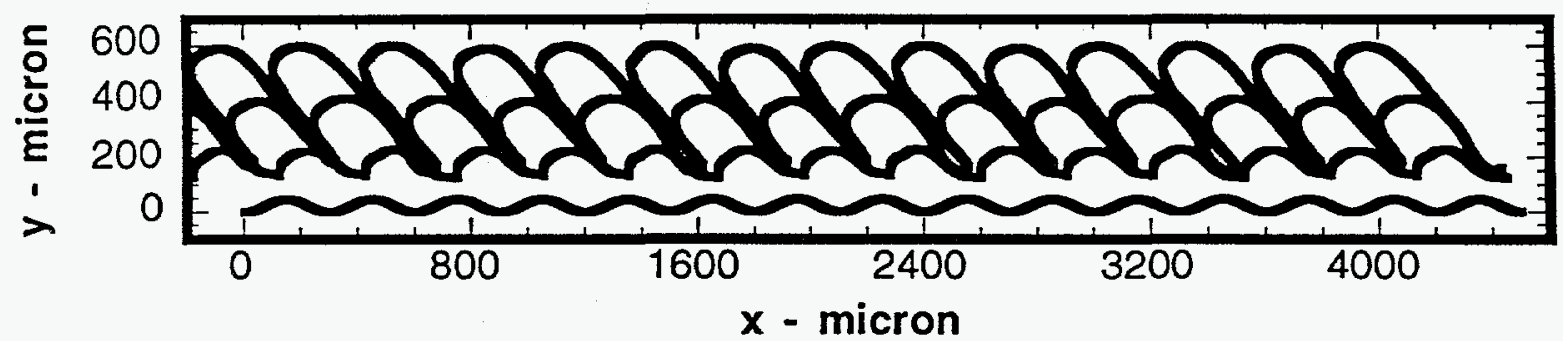

Figure 21a. The shape of the coating surface for a continuous mass flux distribution on a sine wave substrate for $\mathrm{L}=120$ microns, $\theta=26^{\circ}$ and $S_{m 0}=300$ microns (Stable Scallop Regime).

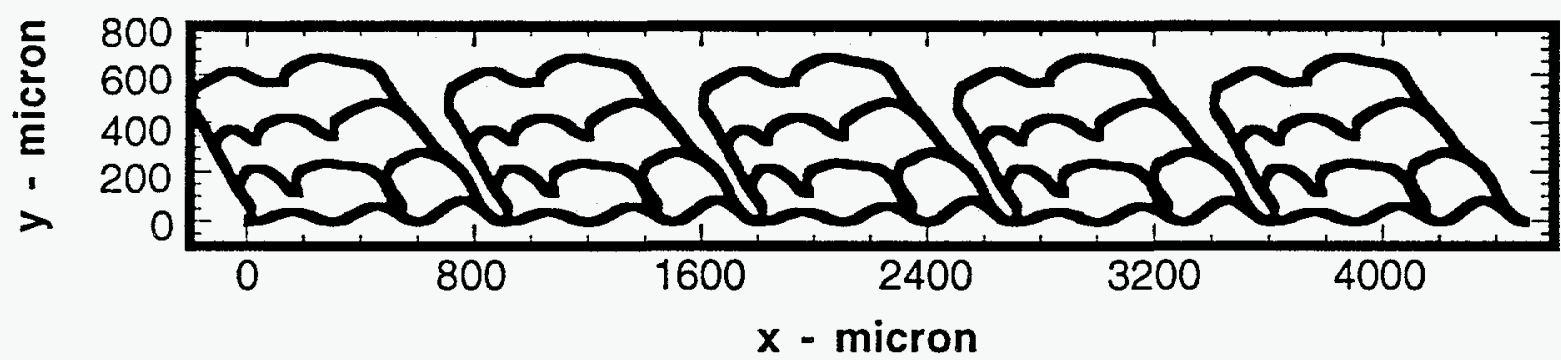

Figure 21b. The shape of the coating surface for a continuous mass flux distribution on a sine wave substrate with staggered amplitudes within each group of three wavelets for $L=120$ microns, $\theta=26^{\circ}$ and $\mathrm{S}_{\mathrm{m} 0}=300$ microns (Stable Scallop Regime).

Although the relationship between scallop spacing, $S_{\mathrm{m}}$, and the splat size, $\mathrm{L}$, and spray angle, $\theta$, established above based on the assumptions of continuous mass deposition and simple profiles for the pre-roughened surface does not strictly apply to real coatings, this relationship is qualitatively correct. That is, in general, $S_{\mathrm{m}}$ increases with splat size, which increases with droplet size. Also, $S_{\mathrm{m}}$ increases with decreasing $\theta$. These results help in interpreting some of the results presented later in this report.

\section{V.2. Stochastic Droplet Deposition}

The results presented in this section were obtained using a simulation for the stochastic deposition of thermal spray droplets onto the substrate. The spray angle was assumed to have a single constant value of, $\theta=26^{\circ}$, and a droplet size, $D$, of 40 microns, was used. A value of three was used for the spread coefficient, $\xi$.

Figure 22 shows the final shape of the coating surface, as well as the surface shape for several intermediate stages, where a smooth substrate was used for the initial condition. In contrast 


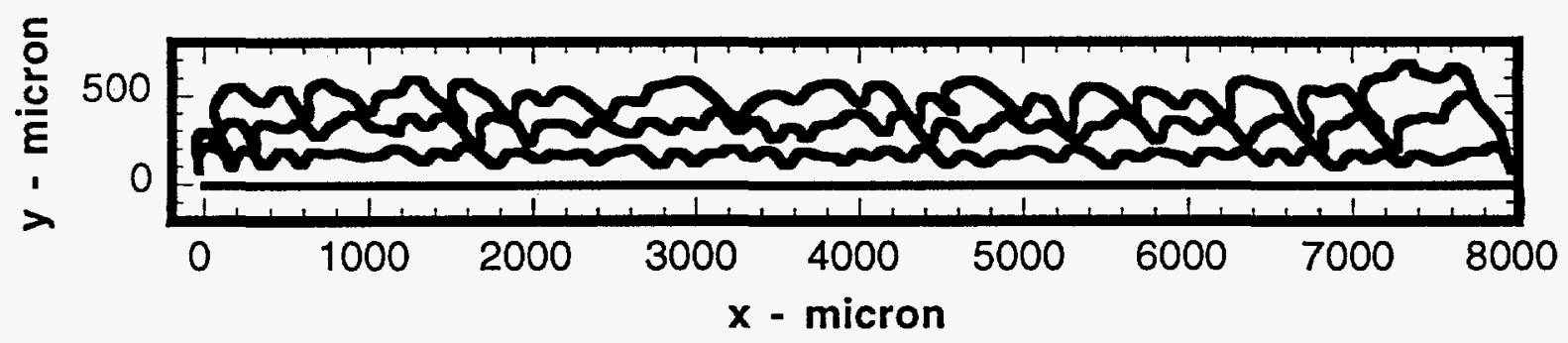

Figure 22. The shape of the coating surface for stochastic droplet deposition on a smooth substrate for $D=40$ microns, $\xi=3$ and $\theta=26^{\circ}$.

to the smooth coating surface obtained using continuous mass deposition onto a smooth substrate, the results obtained using a simulation of the stochastic droplet deposition show that a very rough surface has now developed. This is due to the small roughness elements formed by the first few droplets which were deposited on the substrate. These small roughness elements provide the disturbances which initiate the growth of large scallops. This effect also occurs for stochastic droplet deposition on a pre-roughened substrate, regardless of the value of $S_{\mathrm{m} 0}$. Thus, any attempt to make smooth coatings by using a substrate which does not have disturbances which can initiate scallop growth, e.g. by making $\mathrm{S}_{\mathrm{m} 0}<\mathrm{L}$, will be unsuccessful because of the disturbances created by the discrete thermal spray droplets themselves.

The results for $R_{a}$ and $S_{m}$ for a smooth substrate are shown in Figure 23 as a function of the coating thickness, $y_{m}$, along with results for a rough substrate (profilometer data for the profile of a pre-roughened substrate is used as the initial condition, where $R_{a 0}=9$ microns and $S_{m 0}=148$ microns). Also shown are results for a rough substrate where the variable spread coefficient, $\xi$,
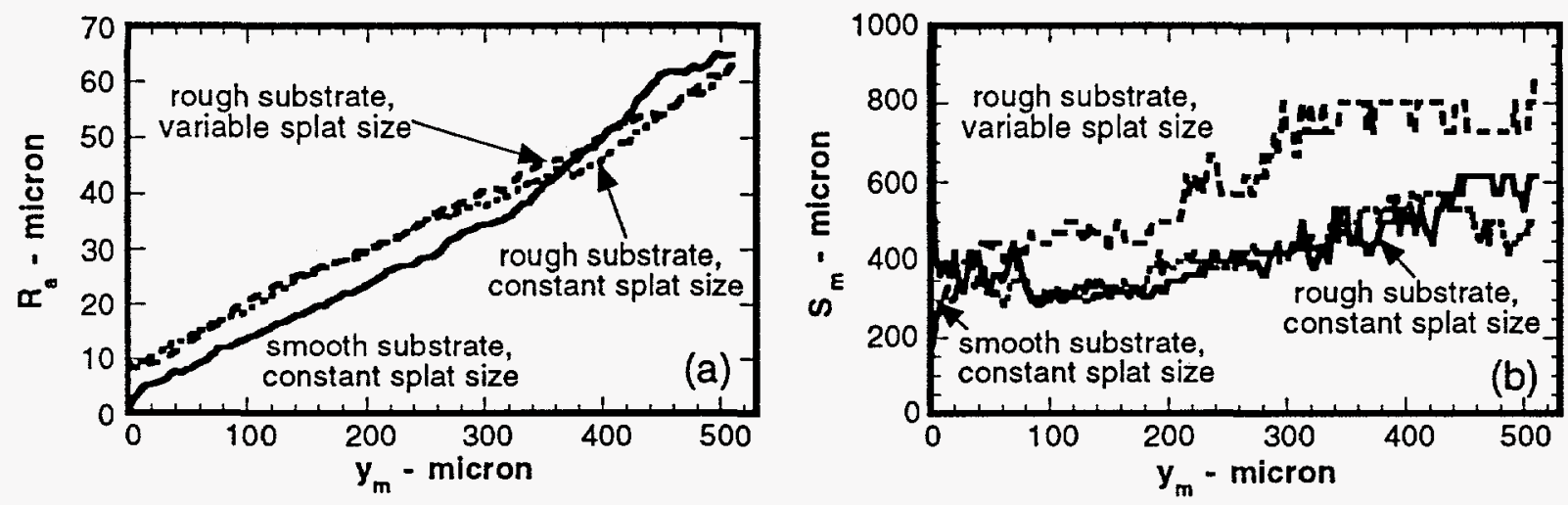

Figure 23. The results for the coating roughness as a function of the coating thickness for stochastic droplet deposition on smooth and rough substrates for $D=40$ microns and $\xi=3$ for constant and variable spread coefficients. (a) $R_{a}$, (b) $S_{m}$.

given in Equation (9) as a function of the local impact angle, $\beta$, was used. All three sets of results show that both $R_{a}$ and $S_{m}$ increase throughout most of the coating process. The results for $R_{a}$ and $S_{m}$ for the smooth and rough substrates are approximately the same. Thus, the differences between the smooth and rough substrates do not appear to be important when the thermal spray mass is composed of droplets which are deposited stochastically. These results for $\mathrm{R}_{\mathrm{a}}$ are also in close agreement with the results for the variable spread coefficient. In contrast, the results for $S_{m}$ for the 
variable spread coefficient are significantly larger than for the constant spread coefficient. This result is consistent with the results obtained using continuous mass deposition on simple surfaces (section V.1), where the stable scallop spacing was found to be proportional to the splat size, i.e. $\mathrm{S}_{\mathrm{m}} \propto \mathrm{L}=\xi \mathrm{D}$. Thus, the larger spread coefficient given by $\xi$ ' results in a larger splat size and a larger value for $S_{m}$.

The results for the final values of $S_{m}$ for the constant spread coefficient, scaled by the splat size ( $L=120$ microns for this case), are approximately 5 and 4 for the rough and smooth substrates, respectively. These values are in the bifurcating scallop regime for the spray angle used here $\left(\theta=26^{\circ}\right)$. These results provide an example where values of $S_{m}$ occur which are larger than the stable values, due to scallop merging resulting from non-uniformities in the coating profile shapes.

\section{V.3. Computed Mass Flux Distribution}

The results presented above are for a single value of the spray angle, $\theta$. The results of Lopez et. al (1995) for droplet trajectories in an axi-symmetric thermal spray jet impinging on a target substrate at a normal angle shows that the droplets do not all strike the target at the same angle. Her results for three different droplet sizes and for droplets with different initial positions in the exit plane of a thermal spray gun, $\mathrm{zg}$ (measured from the center of the exit plane), are shown in Table 1. The results show that droplets with initial positions near the center of the gun exit plane $(\mathrm{zg}=0)$

\begin{tabular}{|c|c|c|c|c|}
\hline $\mathrm{Zg}$ & $\mathrm{N}_{\mathrm{D}}$ & \multicolumn{3}{|c|}{$\theta$ - degrees } \\
\hline Normalized & Normalized & $\mathrm{D}=10 \mu \mathrm{m}$ & $\mathrm{D}=40 \mu \mathrm{m}$ & $\mathrm{D}=80 \mu \mathrm{m}$ \\
\hline 0 & 0.0686 & 90 & 90 & 90 \\
\hline 0.125 & 0.0711 & 89.2 & 89.2 & 89.2 \\
\hline 0.250 & 0.0737 & 88.6 & 88.6 & 88.6 \\
\hline 0.375 & 0.0790 & 87.7 & 87.7 & 87.7 \\
\hline 0.500 & 0.0844 & 86.6 & 86.6 & 86.6 \\
\hline 0.625 & 0.0663 & 84.6 & 84.9 & 84.9 \\
\hline 0.750 & 0.0482 & 81.0 & 81.4 & 81.4 \\
\hline 0.875 & 0.0304 & 74.9 & 77.0 & 77.0 \\
\hline 1.000 & 0.0127 & 65.0 & 71.0 & 71.0 \\
\hline
\end{tabular}

Table 1. The droplet impact angle and number density for a droplet flux distribution (the distribution is symmetric about $\mathrm{z}_{\mathrm{g}}=0$; only one half is shown).

strike the target at a more perpendicular angle than droplets with initial positions near the edge of the gun exit plane $(\mathrm{zg}=1)$. Also shown in Table 1 are experimental results for the droplet number density distribution, $N_{D}$, along the exit plane of a thermal spray gun [Roemer, 1994]. These results show that there are more droplets near the center of the gun exit plane than near its edge. The measured results for the droplet number density are used to weight the computed results for the droplet impact angles to obtain a droplet mass flux distribution which was used to calculate coating roughness. This distribution was assumed to be symmetric about $\mathrm{zg}=0$. (Only one half of 
the distribution used in the calculations is shown in Table 1.) This flux distribution was swept over the target (i.e. to simulate gun stroking) for 40 cycles.

Figure 24 shows the shape of the coating surfaces for $D=10,40$ and 80 microns. The results for $\mathrm{R}_{\mathrm{a}}$ are 8,14 and 30 microns, for $\mathrm{D}=10,40$ and 80 microns, respectively. Although the impact angles for $D=10$ microns are smaller (more off-normal) than for $D=40$ and 80 microns, $R_{a}$ is the smallest for $D=10$ microns. The impact angles shown in Table 1 are very large for all the values for $D$, and this appears to have reduced the effect that the small differences between the angles for $\mathrm{D}=10$ microns and 40 microns (or 80 microns) might have otherwise had on the coating roughness. The primary parameter affecting the roughness results appears to be the droplet size, where $R_{a}$ increases with increasing $D$.

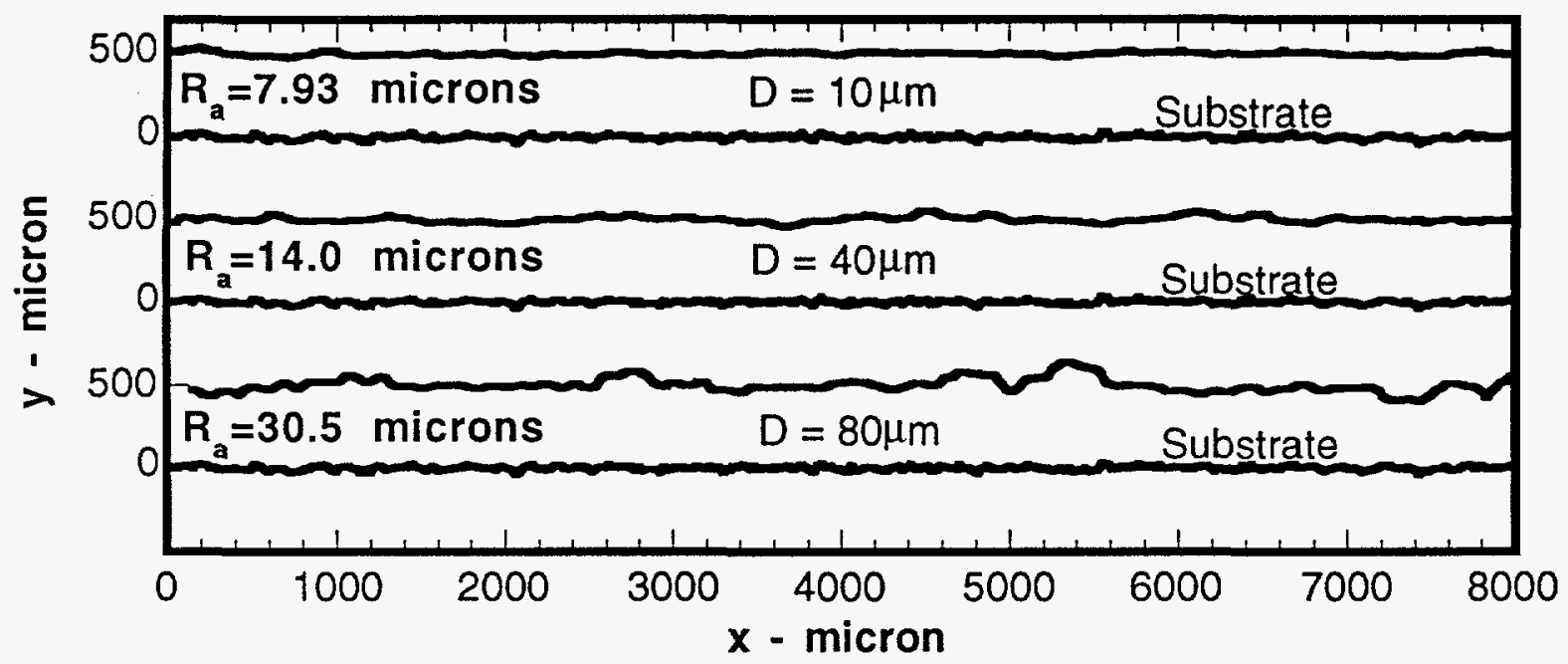

Figure 24. The shapes of the coating surfaces for stochastic droplet deposition onto a rough substrate for calculated droplet trajectories transported by an axi-symmetric gas jet for $\mathrm{D}=10,40$ and 80 microns. 


\section{V.4. Empirical Mass Flux Distribution}

The mass flux shown in Figure 5, which is based on experimental data, is composed of two basic parts, the main spray and the over-spray. The spray angles in the over-spray portion are very small, a characteristic that was not considered in the calculations presented above. This mass flux distribution, which specifies not only the spray angles, but also the fraction of the mass deposited for each spray angle and the droplet size for each spray angle, was used to obtain the results presented in this section. The number of gun strokes over the target was assumed to be 55 . Profilometer data for the profile of a pre-roughened substrate were used as the initial condition.

Figure 25a shows the results for the shape of the coating surface. Although most of the thermal spray mass $(77 \%)$ is deposited at a relatively large spray angle $\left(\theta=51^{\circ}\right)$ the small spray angles for the over-spray is important and results in the development of some large scallops and a very rough surface. The results from the calculations are compared to experimental data for the profile of a coating, shown in Figure 25b. A laser profilometer was used to measure this profile, which scans the coating surface vertically from above; consequently, it is not capable of resolving 'over-hanging' features of the scallops, as obtained in the calculations. There is, however, good agreement between the computed and measured coating profiles for the general size and shape of the scallops.

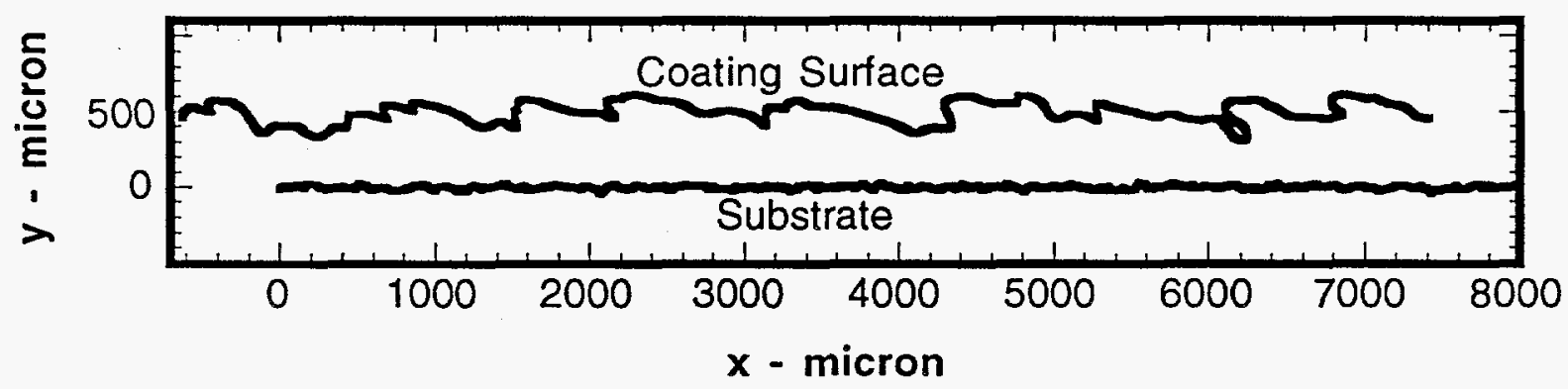

Figure 25a. The calculated shape of the coating surface for the empirical mass flux distribution.

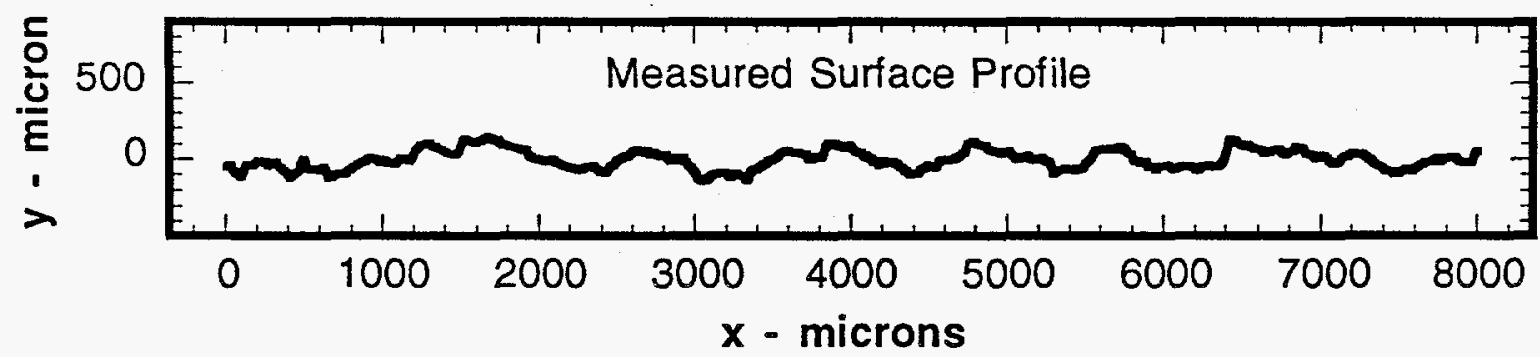

Figure 25b. The measured shape of the coating surface.

Results for $R_{a}$ and $S_{m}$ are presented as a function of the coating thickness, $y_{m}$, in Figure 26. Figure 26a shows $y_{m}$ as a function of time. The growth rate of $y_{m}$ is approximately constant, although this growth rate does vary; e.g. the growth rate is larger during times when the target is exposed to the main spray which has a larger mass flux than that of the over-spray. This is difficult to see in the figure due to the high frequency with which the flux changes back and forth between 
the main spray and the over-spray portions due to the large stroking velocity of the gun. Figures $26 \mathrm{~b}$ and $\mathrm{c}$ show the results for $\mathrm{R}_{\mathrm{a}}$ and $\mathrm{S}_{\mathrm{m}}$, both of which increase throughout the coating process. Figure $26 \mathrm{~d}$ shows the ratio, $\mathrm{R}_{\mathrm{a}} / \mathrm{S}_{\mathrm{m}}$, which is interpreted as an average value for the scallop aspect ratio. Unlike $R_{a}$ and $S_{m}, R_{a} / S_{m}$ comes to a near steady value following an initial development period. The initial values for $R_{a}$ and $S_{m}$, i.e. the values for the pre-roughened substrate, are 9 microns and 148 microns, respectively, giving $\mathrm{R}_{\mathrm{a}} / \mathrm{S}_{\mathrm{m}}=0.061$. During the deposition of the first few microns of material $R_{a}$ increases by a small amount while $S_{m}$ rapidly increases to 258 microns, resulting in a decrease in $R_{a} / S_{m}$ to a value of $0.04 . R_{a} / S_{m}$ then increases to a value of about 0.06 when the coating thickness is 100 microns and varies about that value for the remainder of the coating process. Thus, it appears that if a sufficient amount of material is deposited $R_{a} / S_{m}$ will vary about a constant value, which is 0.06 for the mass flux distribution used here. This condition is referred to here as a 'fully developed' condition.
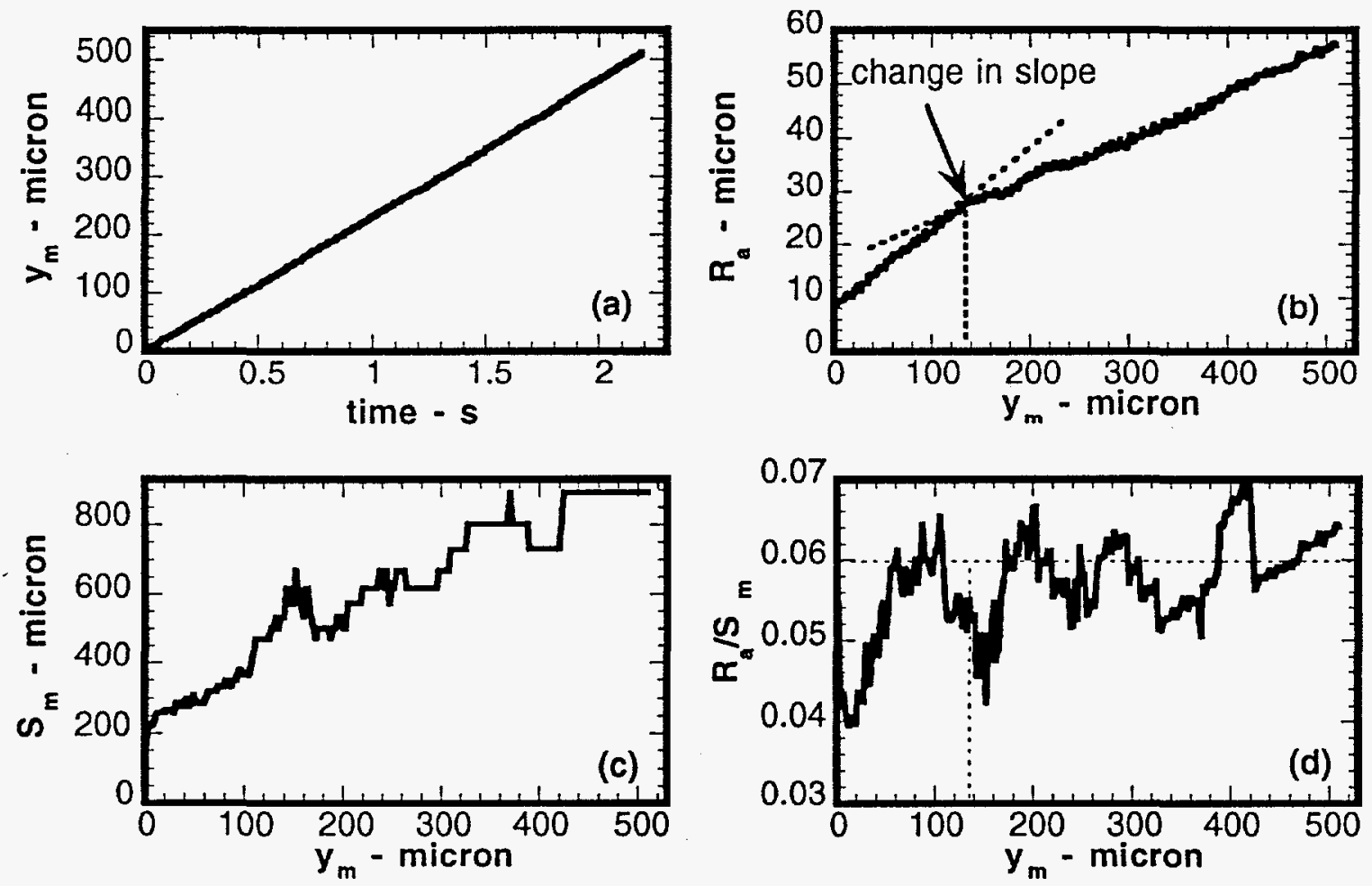

Figure 26. Calculated results for the coating roughness for the empirical mass flux distribution. (a) Coating thickness, $y_{m}$. (b) $R_{a}$, (c) $S_{m}$ and (d) $R_{a} / S_{m}$.

The rate of change of $R_{a}$ with coating thickness decreases when $R_{a} / S_{m}$ is close to the value of 0.06 , i.e. near a coating thickness of 100 microns. Thus, the rate of increase of $R_{a}$ with coating thickness appears to depend on $R_{a} / S_{m}$ and is larger when $R_{a} / S_{m}$ is less than its fully developed value. Recall that the initial value for $R_{a} / S_{m}(0.061)$ was close to its fully developed value, but that it rapidly decreases to a smaller value (0.04) due to the rapid increase in $S_{m}$ for $0<y_{m}<10$ microns (see Figure 26c). This rapid increase in $S_{m}$ occurs when the first few droplets are deposited which cover the pre-roughened surface with relatively large splats. It is also possible that numerical diffusion contributes to this increase in $\mathrm{S}_{\mathrm{m}}$. This numerical diffusion results from the redistribution of the node points which is done at every time step to obtain equal spacing between the node points. 


\section{V.4.1. Comparison with experimental data}

A set of experiments was done where coatings of various thicknesses were made on preroughened cylinders. A laser profilometer measured the profiles of the coatings along lines one inch in length (parallel to the cylinder axis) at several locations on each coating. The roughness of the coatings was determined from the profile data. Figure 27 a shows the experimental results for $\mathrm{R}_{\mathrm{a}}$. As discussed in Appendix A, the droplet sizes in the mass flux distribution used in the calculations were adjusted to fit the experimental data shown in Figure 27; hence the close agreement between the calculated and measured results. The experimental results do confirm that both $R_{a}$ and $S_{m}$ increase throughout the coating process, and that $R_{a} / S_{m}$ attains a fully developed condition'. They also show what appears to be a change in the growth rate of $R_{a}$ near $y_{m}=170$ microns. However, the experimental data does not show the large decrease in $R_{a} / S_{m}$ at the beginning of the coating process which was seen in the calculated results. The reason for this discrepancy is not clear.
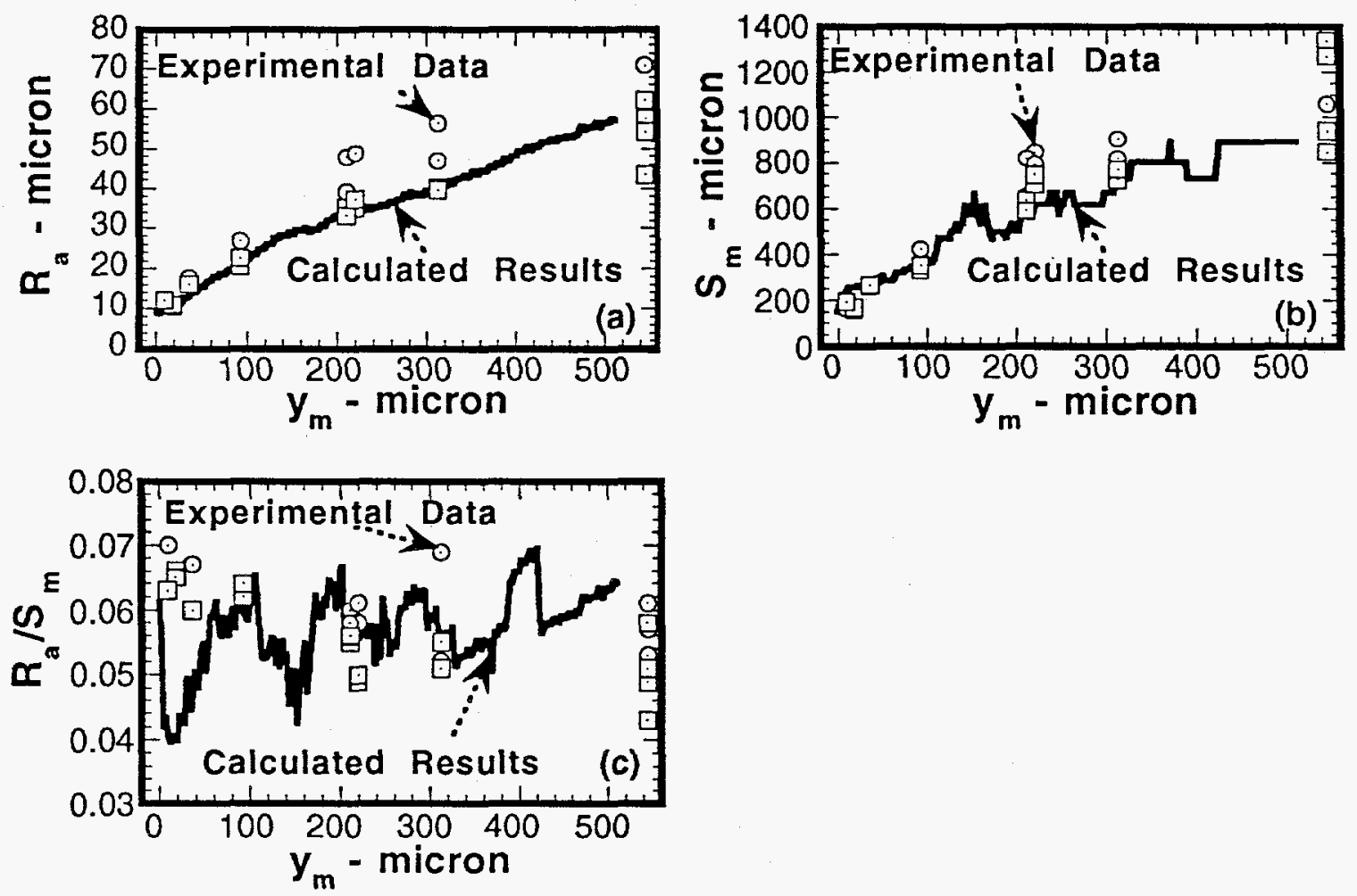

Figure 27. Comparison of calculated and measured results for the coating roughness as a function of the coating thickness, $y_{m}$. (a) $R_{a}$. (b) $S_{m}$. (c) $R_{a} / S_{m}$.

\section{V.4.2 Parameter study}

The calculations were used in a parameter study to determine the sensitivity of the coating surface roughness to the parameters. The parameters considered were the shape of the preroughened substrate profile, the droplet size, the splash fraction (the fraction of the mass deposited by the over-spray portion of the mass flux distribution) and the spray angle for the over-spray. For each parameter varied, the results are compared to the results shown above, which are referred to as the 'standard' results and the values used to obtain the standard results are referred to as the standard parameter values. The standard parameter values are the mass flux distribution parameter 
values shown in Figure 5 for the droplet size (which consists of a set of values for the main spray and over-spray), the splash fraction (standard value $=0.236$ ), and the spray angles for the overspray (which consists of a set of values for the five zones making up the over-spray portion of the mass flux distribution). The profilometer data for the profile of an actual pre-roughened substrate $\left(R_{\mathrm{a} 0}=9\right.$ microns, $S_{\mathrm{m}}=148$ microns) is the standard pre-roughness profile.

Given the relationship identified above between the growth rate of $R_{a}$ and the value of $R_{a} / S_{m}$, it would appear that one method for reducing $\mathrm{R}_{\mathrm{a}}$ is to choose an initial condition, i.e. the preroughness, such that its value for $R_{a} / S_{m}$ is equal to the fully developed value, and its value for $S_{m}$ is large enough to prevent the rapid increase in $S_{m}$ shown in the standard results. This would then eliminate the period of rapid growth of $R_{a}$ associated with small values of $R_{a} / S_{m}$ which occur for small $y_{m}$ in the results for standard conditions. Such a profile for the pre-roughness was obtained by using a sine wave for the initial pre-roughness distribution with an amplitude and wavelength such that $R_{a} / S_{m}=0.06$. (Note that the relationship between the roughness of a sine wave, $R_{a}$ (sine), and its amplitude, $A$, is $R_{a}($ sine $)=2 A / \pi$. Also, $S_{m}$ is the same as the wavelength of a sine wave.) Computed depostion on a smooth substrate was also considered for comparison. Figure 28 shows the results from these calculations, along with results for the standard conditions.
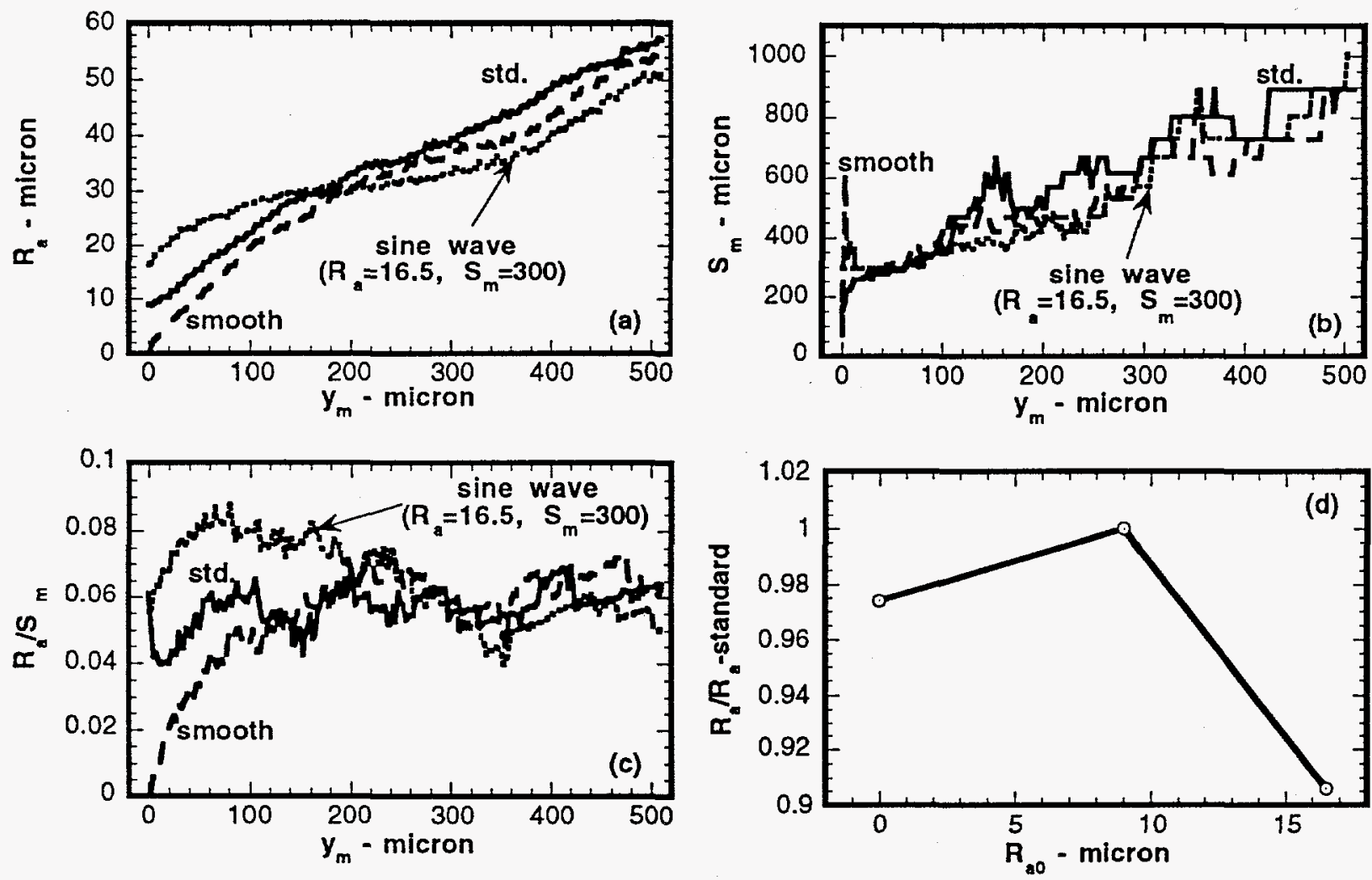

Figure 28. The effect of the pre-roughness profile shape on the coating roughness. (a) $\mathrm{R}_{\mathrm{a}}$. (b) $S_{m}$. (c) $R_{a} / S_{m}$. (d) Final value for $R_{a}$ normalized by the standard result.

The differences between the final results for $R_{a}$ shown in Figure $28 \mathrm{a}$ for the three pre-roughness profiles considered are all small. However, the growth rate of $R_{a}$ for the sine wave pre-roughness profile is significantly smaller than for the other cases. This can be seen in Figure $28 \mathrm{a}$ by noting that the difference between the final and initial roughness values for case using a pre-roughened surface consisting of a sine wave ( $\Delta R_{a}=34$ microns) is significantly less than the results for the 
standard and smooth pre-roughened surfaces $\left(\Delta R_{a}=49\right.$ and 54 microns, respectively). The slower growth rate for $R_{a}$ is due to the larger values for $R_{a} / S_{m}$ (see Figure $28 \mathrm{c}$ ) for the sine wave preroughness profile for small $\mathrm{y}_{\mathrm{m}}$ than it is for the other cases. Unfortunately, the benefit of this smaller growth rate is partially off set by the larger initial value for $R_{a}$ for the sine wave preroughness profile. The results for $\mathrm{S}_{\mathrm{m}}$, shown in Figure $28 \mathrm{~b}$, are all approximately the same. Figure $28 \mathrm{~d}$ shows the final results for $\mathrm{R}_{\mathrm{a}}$ (normalized by the standard result) as a function of the initial value for $R_{a}$. Note that the smallest final value for $R_{a}$ was obtained for the largest initial value for $R_{a}$.

The effect of droplet size on $R_{\mathfrak{a}}$ is shown in Figure 29a. As shown in Figure 5, the thermal spray mass flux is composed of six zones with different droplet sizes. The results presented in
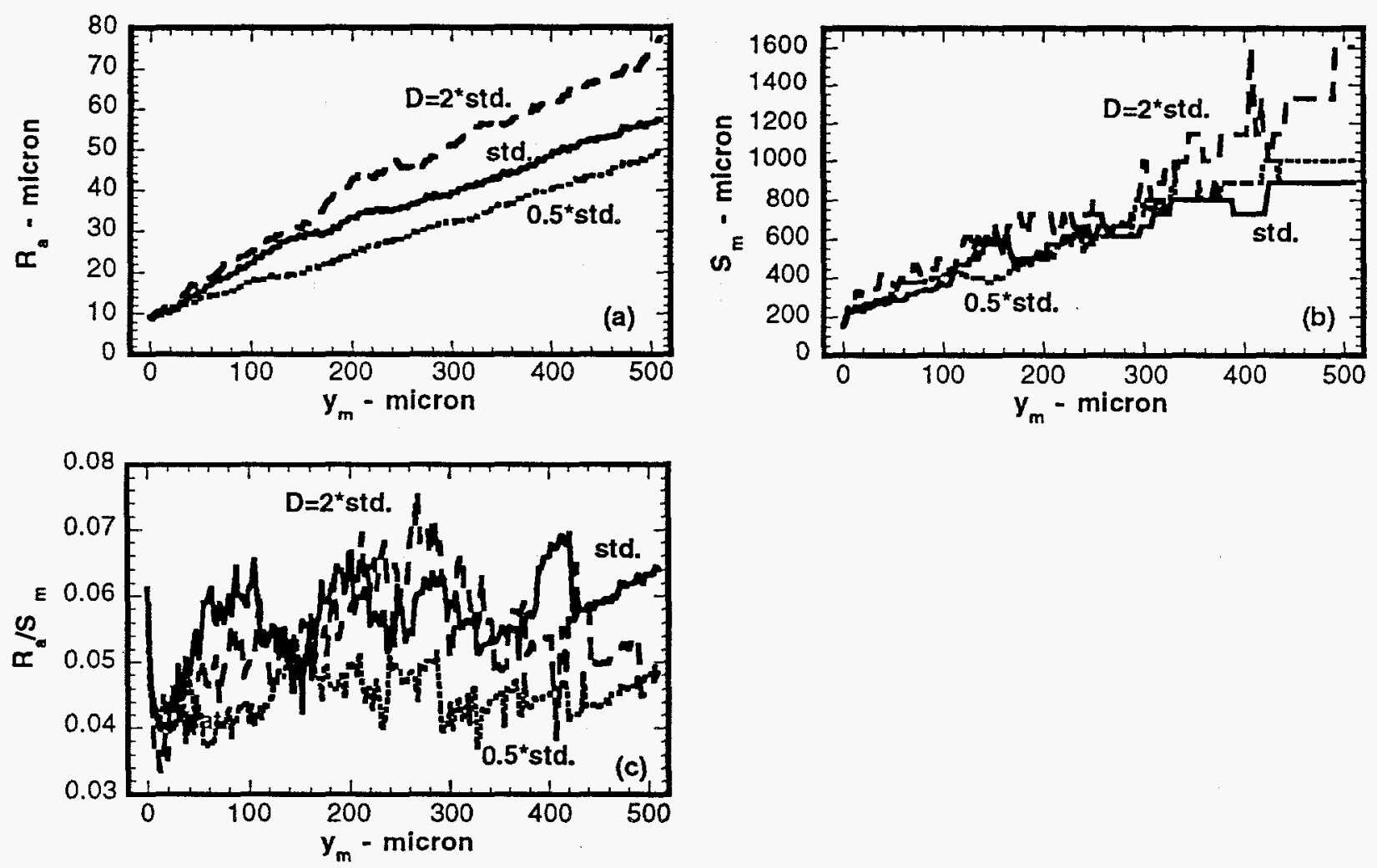

Figure 29. The effect of the droplet size on the coating roughness. (a) $R_{a}$. (b) $S_{m}$. (c) $R_{a} / S_{m}$.

Figure 29 were obtained from calculations where the set of droplet sizes were all scaled by the same factor indicated in the figure. As shown in Figure $29 a, R_{a}$ increases with the droplet size. Figure $29 \mathrm{~b}$ shows that the effect of droplet size on $\mathrm{S}_{\mathrm{m}}$ is small. This is contrary to the results for continuous mass deposition (section V.1), which showed that $S_{m}$ was proportional to the splat size (which is proportional to the droplet size). Those results were for a constant spray angle, while the results here are for a variable spray angle, including very small angles. It appears that the small spray angles used here are the dominating factor in determining $S_{m}$. The decrease in $R_{a}$ may be due to a mass deposition which is more spatially uniform which occurs when $\mathrm{D}$ is decreased, i.e. as $\mathrm{D}$ decreases the number of droplets deposited increases for a given coating thickness. The larger number of droplets are likely to have a more uniform distribution of impact points .

Note that the spread coefficient, splash fraction and all other characteristics of the mass flux were kept constant for the different droplet sizes considered. In reality, any method of altering the 
droplet size would probably also alter some of the other mass flux characteristics, such as the splash fraction. Due to the lack of knowledge of the specific relationship between droplet size and splash fraction for molten metal droplets, this effect was not included in the calculations.

The effect of the splash fraction, $\mathrm{f}_{\mathrm{s}}$, is shown in Figure 30 . As shown in Figure 5 , the mass flux distribution is composed of six zones, where one of these represents the main spray portion of the mass flux, and the remaining five zones represent the over-spray portion of the mass flux. In the standard case described earlier, the sum of the deposit mass fractions for the five zones making up the over-spray portion of the mass flux is $23.4 \%$, i.e. the splash fraction is 0.234 for the standard case. The result labeled ' $0.75^{*}$ std.' is for a mass flux distribution similar to the standard case except that the individual mass flux magnitudes for each of the zones making up the overspray portion of the mass flux were all reduced by a factor of 0.75 such that their sum was 0.175 (i.e. $f_{s}=0.175$ ), and the mass flux magnitude of the main spray was increased to 0.825 . Similarly for the result labeled ' $0.5^{*}$ std.'. The droplet sizes and spray angles of each of the zones in the mass flux distribution were not changed. Figure 30a shows that as the splash fraction decreases, $R_{a}$ decreases. Figure $30 \mathrm{~b}$ shows that $S_{\mathrm{m}}$ also decreases with decreasing $\mathrm{f}_{\mathrm{s}}$. Figure $30 \mathrm{c}$ shows that $\mathrm{R}_{\mathrm{a}} / \mathrm{S}_{\mathrm{m}}$ is much slower to attain its 'fully developed' value as $\mathrm{f}_{\mathrm{s}}$ decreases.
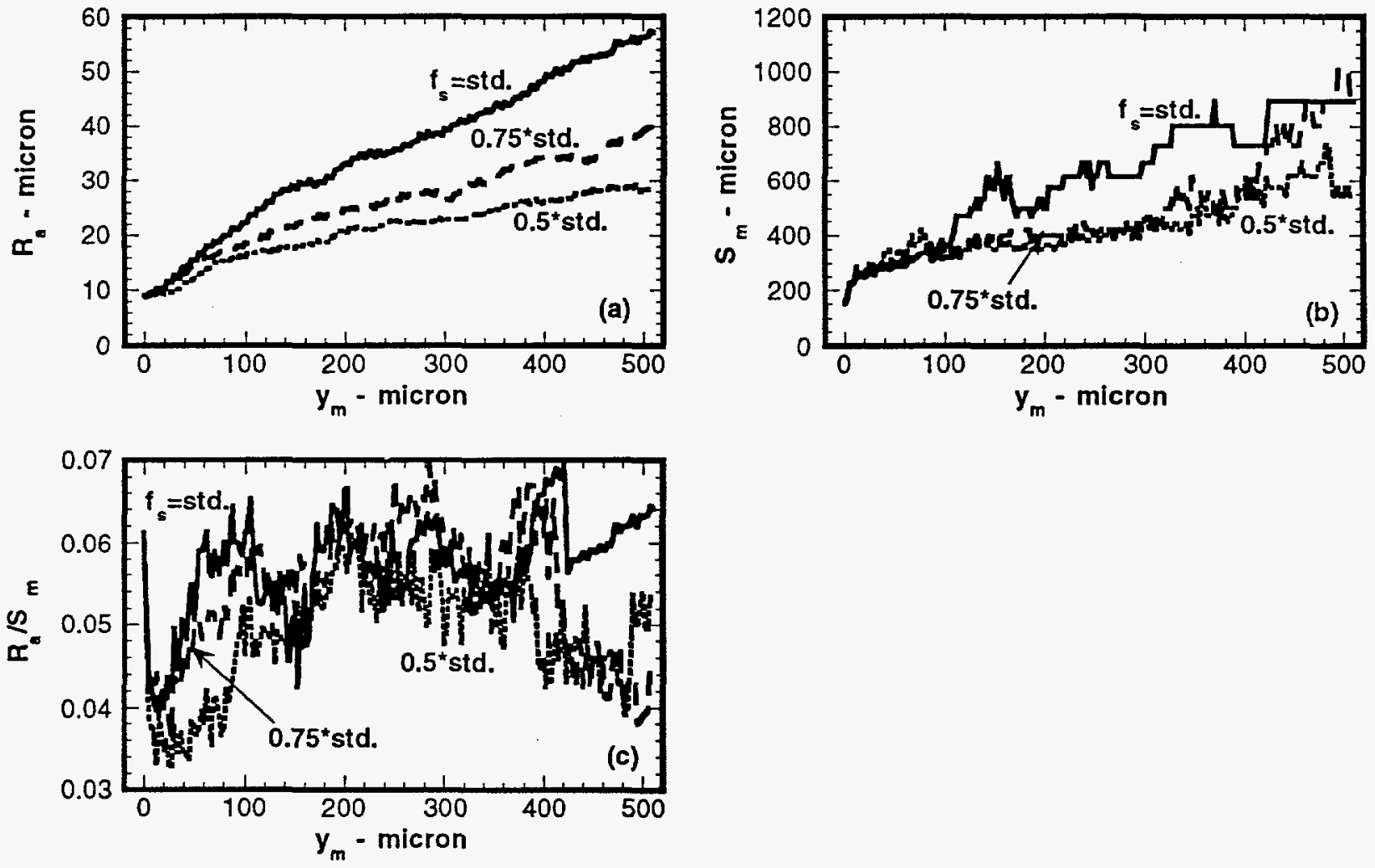

Figure 30. The effect of the splash fraction on the coating roughness. (a) $R_{a}$. (b) $S_{m}$. (c) $R_{a} / S_{m}$.

The effect of the spray angles for the over-spray portion of the mass flux distribution, $\theta_{s}$, is shown in Figure 31. The curve labeled '1.5*std.' is for a mass flux similar to the standard flux except the spray angles for the five zones making up the over-spray portion of the mass flux are all increased by a factor of 1.5. The splash fraction and droplet sizes for the over-spray are all unchanged. Similarly, for the curve labeled ' $2 *$ std.'. The results show (Figure $31 \mathrm{a}$ ) that $\mathrm{R}_{\mathrm{a}}$ is very sensitive to $\theta_{\mathrm{s}}$. Figure $31 \mathrm{~b}$ shows that $S_{\mathrm{m}}$ decreases with increasing $\theta_{\mathrm{s}}$. This is consistent with the results 
presented in section V.1 for continuous mass deposition on simple surfaces, where it was found that the 'stable' value of $S_{m}$ decreases with increasing spray angle. Similar to the effect of $f_{s}$, $\mathrm{R}_{\mathrm{a}} / \mathrm{S}_{\mathrm{m}}$ is much slower to attain its 'fully developed' value for increasing $\theta_{\mathrm{s}}$ (see Figure $31 \mathrm{c}$ ).
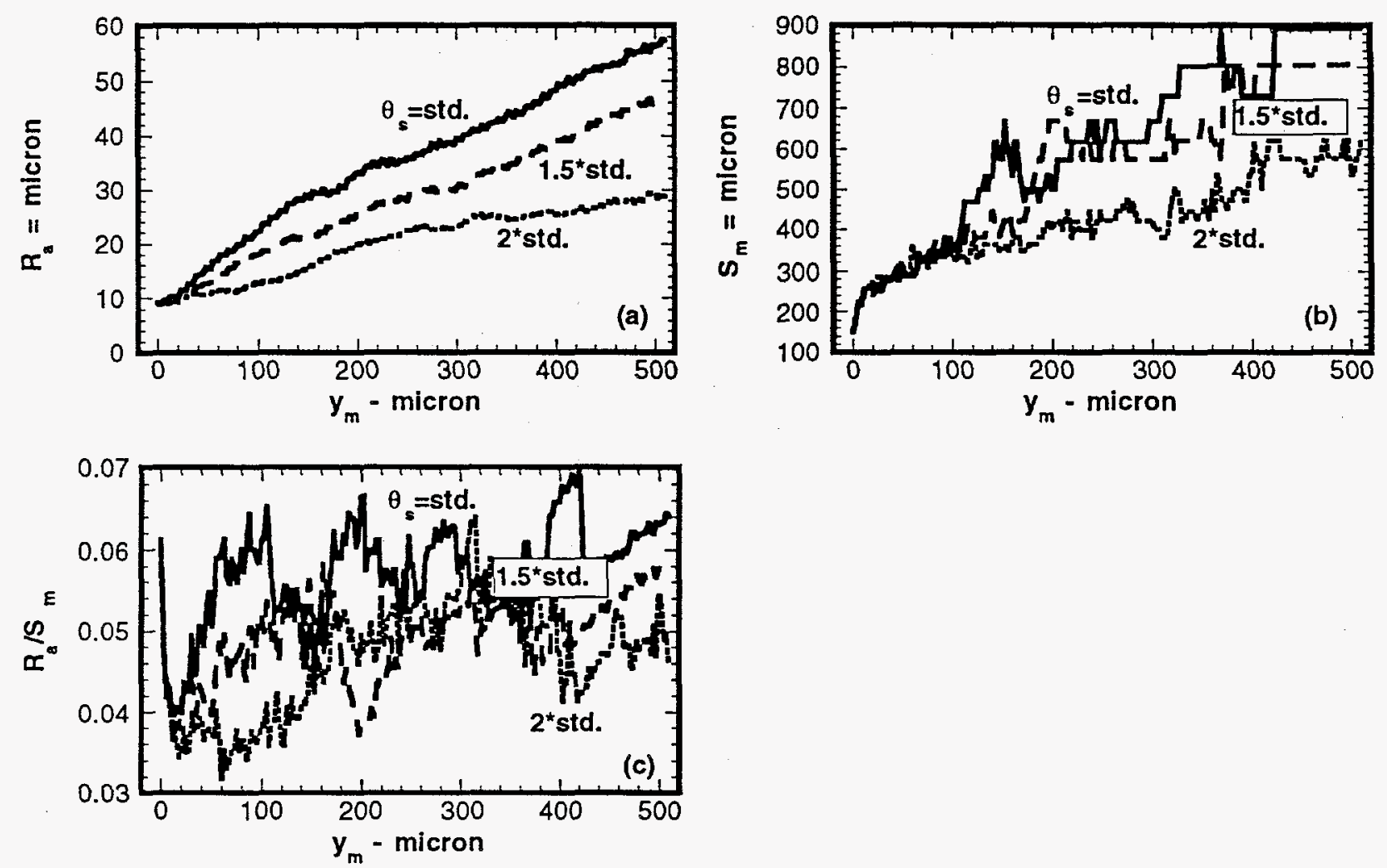

Figure 31. The effect of the spray angle for the over-spray on the coating roughness. (a) $R_{a}$. (b) $\mathrm{S}_{\mathrm{m} \cdot}$ (c) $\mathrm{R}_{\mathrm{a}} / \mathrm{S}_{\mathrm{m}}$.

\section{V.4.3 Porosity}

The analysis provides a prediction for the formation of pores in the coating based on purely geometrical considerations; i.e. when the shape of the coating surface is such that two parts of the surface come into contact with one another it can close off a void, or pore, which cannot be filled in by additional mass deposition. This is not the only mechanism possible for pore formation in thermal spray coatings. Cirolini et. al (1991) (see also Harding et. al, 1995) proposed that pores form when a splat on the coating surface curls up creating a void beneath the edge of the splat. Fukanuma (1994) proposed that pores form when an impacting droplet traps gas between it and the coating surface such that the resulting splat does not completely follow the underlying surface contour. These mechanisms for pore formation are not accounted for here.

One of the factors which has been observed to contribute to large pore formation in the coating is open porosity in the substrate (Campbell, 1996), i.e. existing porosity in the substrate which is partially exposed by the pre-roughening process such that a large hole in the substrate surface is created. The coating process is often unable to fill such holes, and simply seals them off leaving a large open pore buried in the coating. The photo-micrograph shown in Figure 32a is an example of two such pores. 
Calculations were done in an attempt to reproduce the large pores in the photo-micrograph shown in Figure 32a. The portion of the substrate profile visible in the photo-micrograph was digitized and used as the initial condition for the calculations. The results, shown in Figures $32 \mathrm{~b}$ and $c$, predict the formation of one of the large pores in the photo-micrograph. The other large pore did not form in the calculations. Figure $32 \mathrm{c}$ shows the coating surface evolving in the calculation; note that the two parts of the coating surface on either side of the large hole in the substrate first approach one another, then come into contact and seal off the pore.
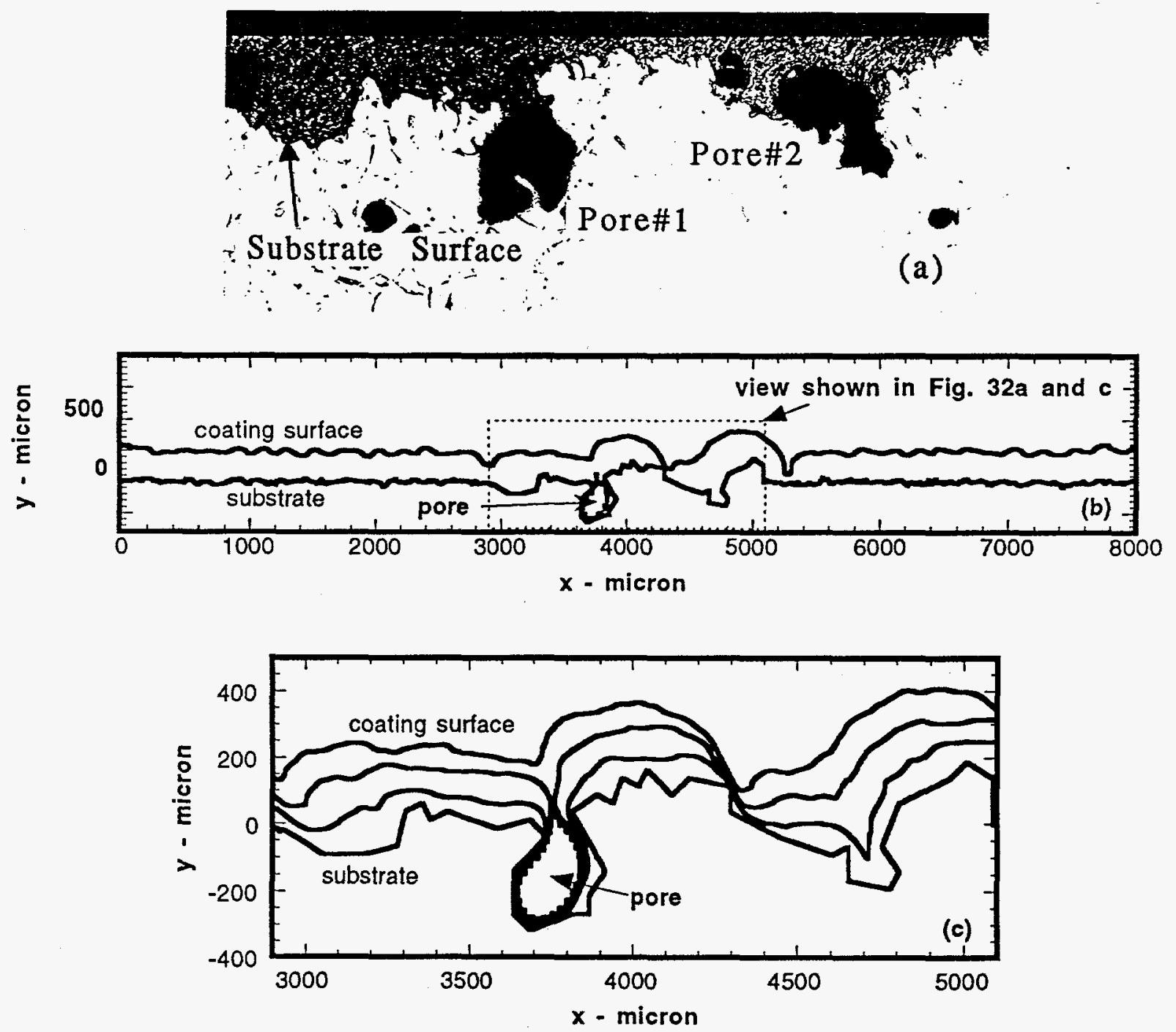

Figure 32. The shape of the coating surface and the shape of a large pore which forms in a hole in the substrate. (a) Photo-micrograph after final machining of the sprayed surface. (b) Calculated result. (c) Enlarged view of the calculated result.

The photo-micrograph shown in Figure 33a is a second example of a large pore forming in a coating on a substrate with a large hole in it. The calculated results are shown in Figure $33 \mathrm{~b}$, which also show the formation of a large pore. This example and the one shown in Figure 32 confirm that the analysis can explain the formation of large pores in the coating due to large holes in the substrate. 

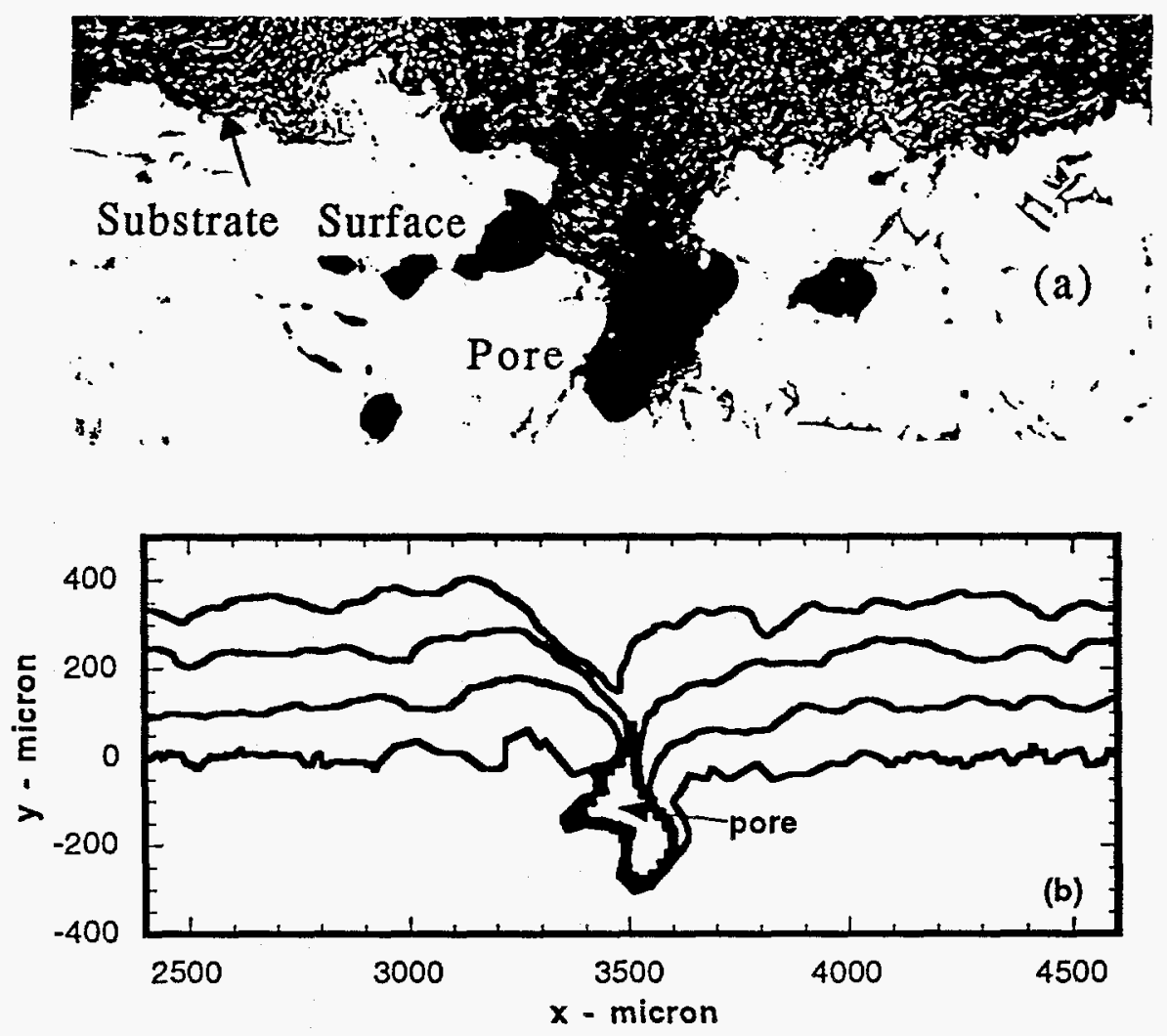

Figure 33. The shape of the coating surface and the shape of a large pore which forms in a hole in the substrate. (a) Photo-micrograph. (b) Calculated result.

Porosity has also been observed in the coatings, even in the absence of large holes in the substrate, although the pores in this case are much smaller than those shown above. Calculations were carried out to appraise the ability to predict the formation of such pores. The calculations used the standard profile for a pre-roughened substrate (without any large holes) as the initial condition, and the thermal spray gun was assumed to traverse over the target for a total of 40 cycles. The computed coating surface was searched for 'positive-loops' after every time step. The results, shown in Figure 34, confirm that the analysis predicts the formation of small pores within the coating. In this case, a total of 36 pores formed. The overall porosity, defined here as the total cross sectional area of all the pores divided by the cross sectional area of the coating, was $0.23 \%$.

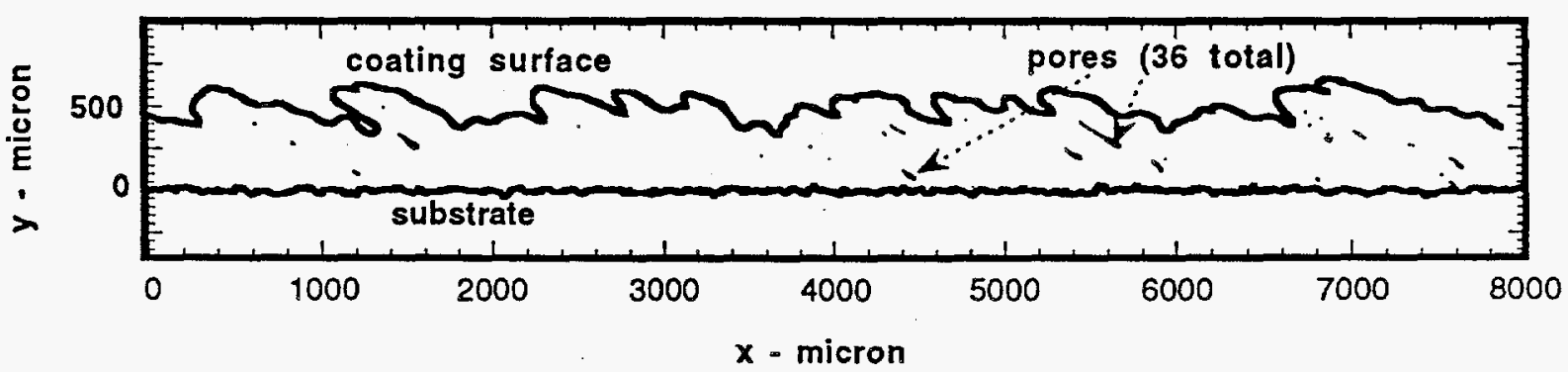

Figure 34. Results for the distribution of porosity in a coating. 


\section{SUMMARY}

The formation of a thermal spray coating has been analyzed to identify methods to reduce the surface roughness of the coating. In developing the analysis, a new methodology was developed. The method uses a string of equally spaced node points to define the shape of the coating surface and tracks the change in this shape as the thermal spray mass is deposited. The method allows for the calculation of arbitrary shapes for the coating surface which may be very complex.

The analysis accounts for the shading which occurs when large roughness elements, called scallops, form on the coating surface. These scallops block the thermal spray from directly depositing on regions of the coating surface adjacent to the scallops. Also included is the spreading that occurs when a thermal spray droplet impacts with the coating surface and spread out over distances that are large relative to the droplet itself.

One of the thermal spray mass flux distributions used for the droplet size, spray angle and mass flux magnitude was based on experimental data. The data show that there are two basic parts to the thermal spray mass flux, the main spray part and the over-spray part. The main spray part of the mass flux is that portion of the mass deposited directly by the thermal spray jet coming from the thermal spray gun. The conditions of this portion of the mass flux include large values for the droplet size and the spray angle. The over-spray part of the mass flux is that portion of the main thermal spray droplets which splashes on impact with the target surface, and is ejected off of the target surface and back into the gas flow field next to the target. The splashed droplets are then transported to other regions of the target surface where they are re-deposited. The deposition conditions of the over-spray include small values for the droplet size and the spray angle. The spatial distributions of the thermal spray droplets in both portions of the spray are random, which results in a stochastic deposition of the droplets onto the target. This stochastic deposition of the droplets is accounted for in the model.

The results from this study have shown that there are two causes of the formation of scallops on the coating surface, both of which must be present. These are 1), a small spray angle (i.e. less than $30^{\circ}$ ), and 2), geometrical features on the target surface which initiate the growth of the scallops; that is, a smooth coating surface is unstable and it remains smooth only if there are no disturbances present in the target surface. Geometrical features in the target surface with a spacing larger than the splat size initiate the growth of scallops. These features may be in the profile of the pre-roughened substrate or they can be created by the thermal spray itself.

The growth of scallops was found to be a complex phenomena. The average height, $R_{a}$, and spacing, $S_{\mathrm{m}}$, of the scallops increases with increasing coating thickness. The increase in scallop spacing can be caused by scallop merging, where two or more scallops merge to become one large scallop. Merging results from either a scallop spacing which is approximately equal to the splat size, or from a non-uniformity in the scallop heights. Small spray angles can also result in an increase in scallop spacing. Following an initial development period of the coating, the ratio of $R_{a} / S_{m}$ was found to come to a steady, 'fully developed' value. The growth rate of $R_{a}$ was found to be larger for values of $R_{a} / S_{m}$ which were less than the fully developed value than when $R_{a} / S_{m}$ was equal to its fully developed value.

A parameter study was carried out to identify methods for reducing the coating roughness. The parameters considered were the shape of the profile for the pre-roughened substrate, the droplet size, the spray angle for the over-spray and the splash fraction (the fraction of the thermal spray mass composing the over-spray). Figure 35 summarizes the results from this parameter study, where the results have been normalized by the value of the coating roughness representative of the current process (the standard value). The effect of the form of the profile for the pre-roughness was found to be small. The largest reduction in coating roughness obtained by modifying this 
profile was $9 \%$ and was obtained from a profile in the shape of a sine wave with a wavelength of 300 microns. Decreasing the droplet size by a factor of two decreased the roughness by $13 \%$. Increasing the spray angle for the over-spray by a factor of two decreased the roughness by $50 \%$, and decreasing the splash fraction decreased the roughness by $51 \%$.
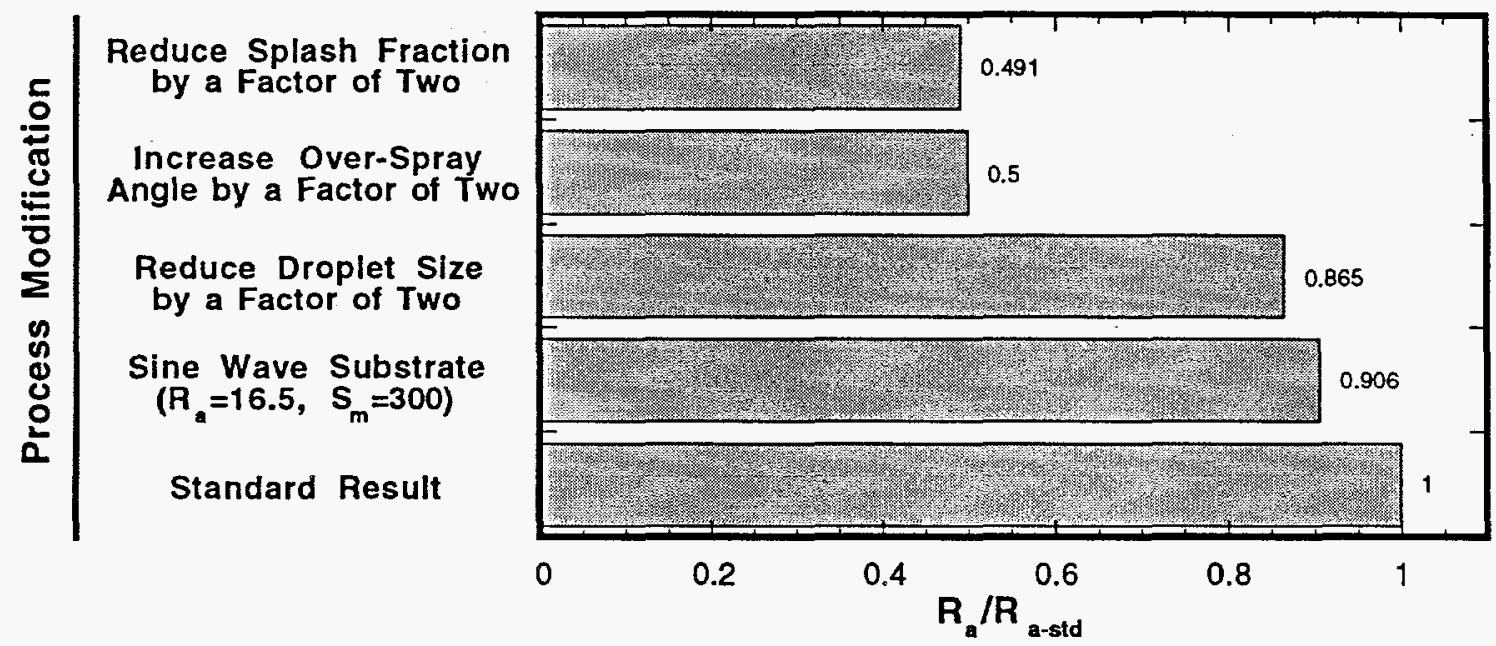

Figure 35. The effect of modifications to the model parameters on the results for coating roughness.

The fraction of a droplet which splashes is determined by the droplet size, impact velocity and angle, the target surface roughness, droplet viscosity, etc.. Thus, reducing the droplet splash fraction requires first, some knowledge of the relationship between these quantities and the splash fraction, and second, a means for controlling these quantities. The spray angle for the over-spray is determined by the gas flow field adjacent to the target surface. This gas flow field may be altered by developing a thermal spray gun which includes a second gas jet as shown in Figure 36 . This conceptual design shows the second gas jet impinging on the over-spray plume containing the splashed droplets, and driving them down onto the target and increasing their spray angle. Alternatively, a second gas jet could be used to blow the over-spray beyond the target surface such that it does not re-deposit, in effect, reducing the splash fraction.

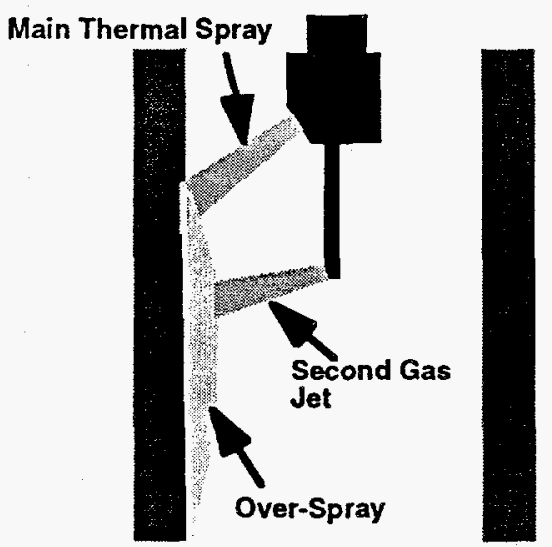

Figure 36. A conceptual design for a new thermal spray gun for increasing the spray angle of the over-spray. 


\section{BIBLIOGRAPHY}

L. Bianchi, F. Blein, P. Lucchese, M. Vardelle, A. Vardelle and P. Fauchais, "Effect of Particle Velocity and Substrate Temperature on Alumina and Zirconia Splat Formation", in Proceedings of the 7th National Thermal Spray Conference, Boston, Massachusetts, (ASM International, Materials Park, Ohio), pp 569-574, 1994.

L. Byrnes and M. Kramer, "Method and Apparatus for the Application of Thermal Spray Coatings Onto Aluminum Engine Cylinder Bores", in 1994 Advances in Thermal Spray Science and Technology, (ASM International, Materials Park, Ohio) pp 39-42, 1994.

R. C. Campbell, private communication, January 1996.

S. Cirolini, J. H. Harding and G. Jacucci, "Computer Simulation of Plasma-Sprayed Coatings. I. Coating Deposition Model", Surface and Coating Technology, 48, pp 137-145, 1991.

J. Domnick, A. Lindenthal, C. Mundo, M. Rüger and M. Sommerfeld, "Combined Experimental and Numerical Investigations of the Spray Coating Process", in Proceedings of the 7th International Coating Process Science \& Technology Symposium. Atlanta, Georgia, edited by xxxx, (ASM International, Materials Park, Ohio), pp 1-20, 1994.

R. C. Dykhuizen, "Review of Impact and Solidification of Molten Thermal Spray Droplets", J. of Thermal Spray Technology, 3, pp 351-361, 1994.

S. Fantassi, M. Vardelle, A. Vardelle and P. Fauchais, "Influence of the Velocity of Plasma Sprayed Particles on the Splat Formation", in Proceedings of the 1993 National Thermal Spray Conference, Anaheim, California, (ASM International, Materials Park, Ohio), pp 1-6, 1993.

H. Fukanuma, "A Porosity Formation and Flattening Model of an Impinging Molten Particle in Thermal Spray Coatings", J. of Thermal Spray Technology, 3, pp 33-44, 1994.

J. H. Harding, P. A. Mulheran, S. Cirolini, M. Marchese and G. Jaccuci, "Modeling the Deposition Process of Thermal Barrier Coatings", J. of Thermal Spray Technology, 4, pp 34-40, 1995.

B. Hassan, A. R. Lopez and W. L. Oberkampf, "Computational Analysis of a Three-Dimensional High-Velocity Oxygen-Fuel (HVOF) Thermal Spray Torch", in 1995 Advances in Thermal Spray Science and Technology edited by C. C. Berndt and S. Sampath, (ASM International, Materials Park, Ohio) pp 193-198, 1995.

A. Hasui, S. Kitahara and T. Fukushima, "On Relation between Properties of Coating and Spraying Angle in Plasma Jet Spraying", Transactions of National Research Institute for Metals, 12, pp 9-20, 1970.

O. Knotek, E. Lugscheider, P. Jokiel, U. Schnaut and A. Wiemers, "Chromium Coatings by HVOF Thermal Spraying: Simulation and Practical Results", in Proceedings of the 7th National Thermal Spray Conference, Boston, Massachusetts, edited by xxxx, (ASM International, Materials Park, Ohio), pp 179-184, 1994.

A. R. Lopez and W. L. Oberkampf, "CFD-ACE Particle Simulations of a $90^{\circ}$ Jet Impinging on a Flat Plate", memorandum to M. P. Kanouff, Sandia National Laboratories, August 1, 1995.

J. Madejski, "Solidification of Droplets on a Cold Surface", Int. J. Heat Mass Transfer, 19 , pp 1009-1013, 1976. 
G. Montavon, C. Coddet, S. Sampath, H. Herman and C. C. Berndt, "Vacuum Plasma Spray Forming of Astroloy: An Investigation of Processing Parameters", in Proceedings of the 7th National Thermal Spray Conference, Boston, Massachusetts, (ASM International, Materials Park, Ohio), pp 469-475, 1994.

C. Moreau, P. Gougeon and M. Lamontagne, "Influence of Substrate Preparation on the Flattening and Cooling of Plasma-Sprayed Particles", J. of Thermal Spray Technology, 4, pp 2533,1995 .

P. A. Mulheran, J. H. Harding, R. Kingswell and K. T. Scott, "Modeling the Structural Development in a Plasma Sprayed Coating", in Proceedings of the International Thermal Spray Conference and Exposition, Orlando, Florida, (ASM International, Materials Park, Ohio), pp 749753, 1992.

R. A. Neiser, J. E. Brockmann, T. J. O'Hern, R. C. Dykhuizen, M. F. Smith, T. J. Roemer and R. E. Teets, "Wire Melting and Droplet Atomization in a HVOF Jet", in 1995 Advances in Thermal Spray Science and Technology edited by C. C. Berndt and S. Sampath, (ASM International, Materials Park, Ohio) pp 99-104, 1995.

R. A. Neiser, Private communication, January 1996.

T. Roemer, "HVOF M2 Exit Aperture Particle Distribution", memorandum to M. P. Kanouff, Sandia National Laboratories, November 30, 1994.

M. Sander, A Practical Guide to the Assessment of Surface Texture, (Fienprïf Perthen GmbH, Göttingen, 1991).

M. F. Smith, R. A. Neiser and R. C. Dykhuizen, "An Investigation of the Effects of Droplet Impact Angle in Thermal Spray Deposition", in Proceedings of the 7th National Thermal Spray Conference, Boston, Massachusetts, (ASM International, Materials Park, Ohio), pp 603-608, 1994.

Sir G. Taylor, "Oblique Impact of a Jet on a Plane Surface", Proc. Roy. Soc. A, 260, pp 96-100, 1961. 


\section{APPENDIX A. THE THERMAL SPRAY MASS FLUX DISTRIBUTION EXPERIMENT}

Figure A1 shows a schematic of the experiment used to study the distribution of mass deposited on a flat plate by a thermal spray gun with an angled air cap. A Metco DJ thermal spray gun with an M2 air cap was used to make the deposit on the plate. The gun was operated in the low BTU condition and steel wire (1/8 inch diameter) was fed at $44 \mathrm{in} / \mathrm{min}$. After gun start-up, the gun traversed across the plate (normal to the plane of Figure A1) at $100 \mathrm{in} / \mathrm{min}$. A total of $15 \mathrm{passes}$ were used to make the deposit. A grit blasted aluminum plate with dimensions 2 in by 8 in by 0.125 in was used as the target.

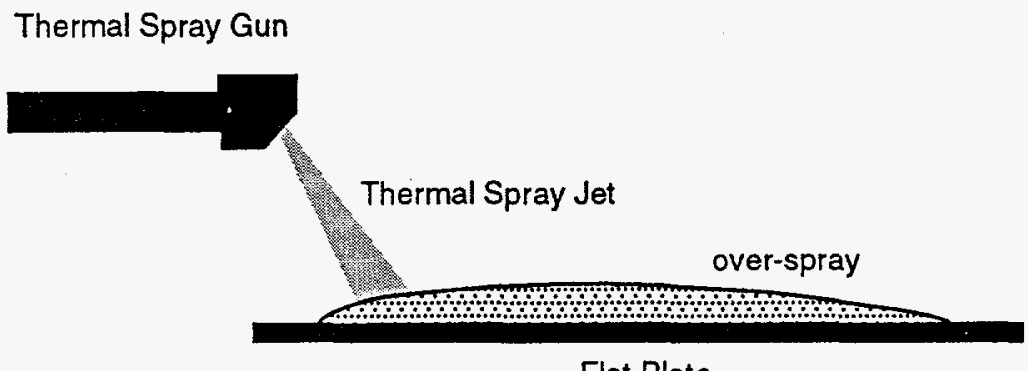

Flat Plate

Figure A1. A sketch of the experimental arrangement used to make a thermal spray deposit on a flat plate. The thermal spray gun traversed across the plate (normal to the plane of the figure).

As discussed in section III.2.1, an infra-red picture (shown in Figure 4) of the experiment showed a long bright image above the plate starting from a location near the impact area of the main thermal spray jet. This image, shown schematically in Figure A1, was formed by the light emitted from the thermal spray mass which had splashed due to the impact of the droplets in the main jet with the plate, where the splashed mass, called over-spray was ejected off the plate and swept downstream (parallel to the plate) by the gas flow. The location and length of this image correlated with the location and length of the deposited mass which was later found on the plate.

The deposit was cut and polished to create a metallurgical sample of the cross section (in the plane of Figure A1) of the deposit which was then photographed. The photograph of the deposit cross section was digitized giving geometric definition of its boundaries. This digitized data, shown in Figure A2, was used to make an estimate of the distribution of mass (i.e. volume of mass) deposited along the plate. The deposit was divided into six zones that could be characterized as locally uniform in terms of the shape and thickness of the deposit. One of the zones covers that portion of the deposit made by the fraction of the mass in the main thermal spray jet which did not splash, and the other five zones in combination cover the over-spray portion of the deposit. The over-spray portion of the deposit was easily distinguished from the main spray deposit because it consisted of isolated columns of material with large gaps in between them. Calculations were done to determine the droplet size and spray angle in each of the over-spray deposit zones by assuming these quantities to be locally uniform and by using a trial and error procedure to identify the values of these input parameters which gave results for the shape of the deposit which compared with the results from the experiment. The calculated results for the width of the columns and the distances between them were found to be sensitive to the droplet size and the spray angle used.

Figures $2 b$ through $2 \mathrm{e}$ show calculated results for the shape of the deposit in comparison to the experimental results for the different zones along the plate. The comparison is good for the overall width of the columns and the distances between them. The spray angle for the main spray deposit (zone 2) is $51^{\circ}$, which is based on video images of the main spray (see Fig. 4). The droplet size for the main spray deposit is 40 microns, which is based on the measurements by Neiser, 1996. 

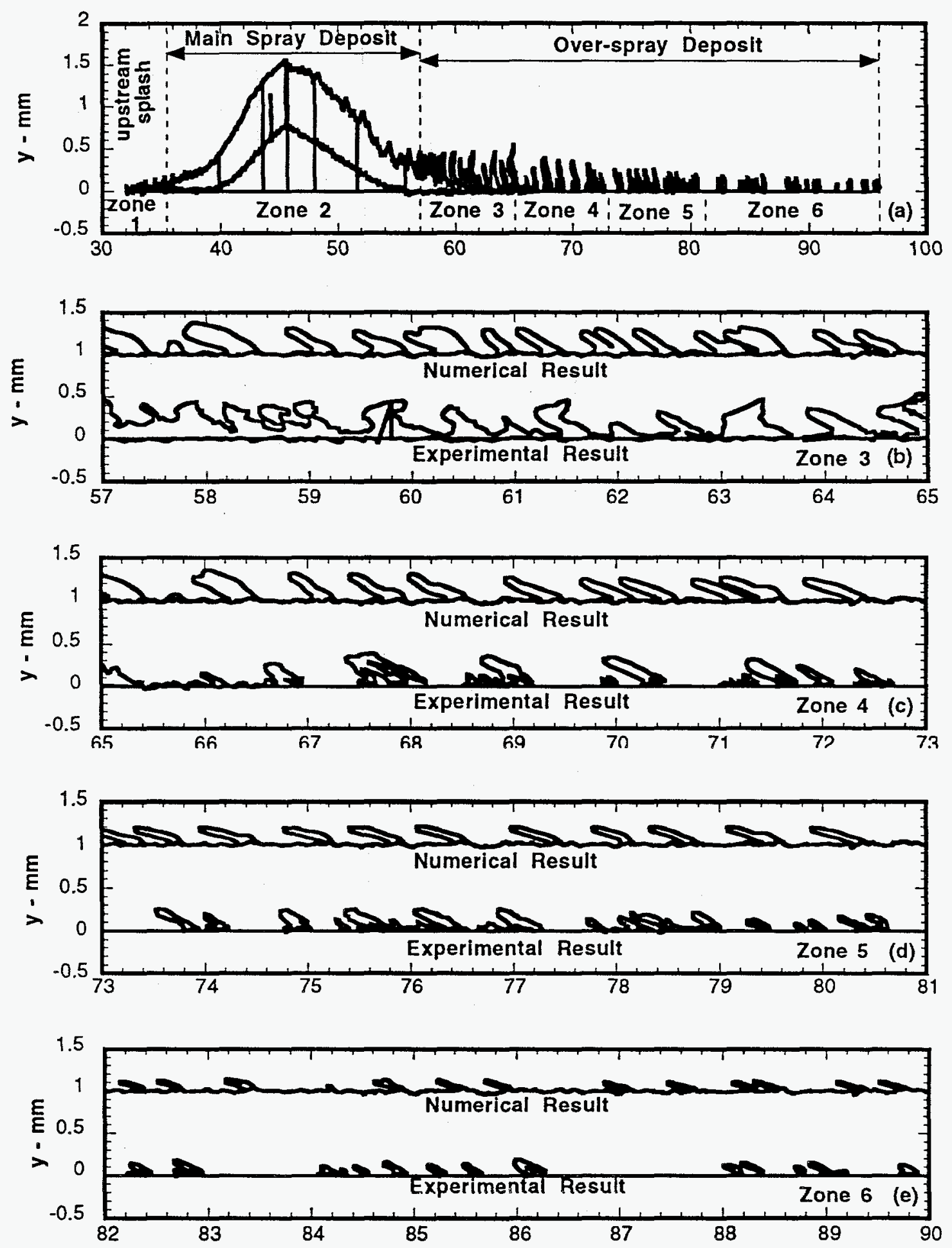

Figure A2. Comparison of numerical and experimental results for the shape of the deposit formed on a flat plate. The digitized experimental data of the deposit cross section (a), and both numerical and experimental results for zone $3(\mathrm{~b})$, zone 4 (c), zone 5 (d) and zone $6(\mathrm{e})$. 
The final results for the distribution of the flux magnitude, the droplet size and the spray angle are shown in Figure 5 in section III.2.1. This experiment showed that the thermal spray mass is deposited over a large length of the target and that the deposition conditions (spray angle and droplet size) vary by large amounts over this length of target.

The distributions for the mass flux magnitude, droplet size and spray angle found by the procedure described above, were used to calculate the roughness of a coating as a function of the coating thickness. The results from these calculations are shown in Figure A3 (labeled, 'Original $\mathrm{D}_{\mathrm{s}}{ }^{\prime}$ ), along with experimental data for the coating roughness on cylinders. The calculated results for the average scallop spacing, $S_{m}$, were in good agreement with the data, but the calculated results for the coating roughness, $R_{a}$, and the ratio, $R_{a} / S_{m}$, were significantly larger than the experimental results. The conditions for the gas flow rates and exhaust vent used in the flat plate experiments used to determine the mass flux distribution were different than those used in the cylinder experiments used to determine the coating roughness. It is possible that these differences resulted in different mass flux distributions. Since the conditions used in the cylinder experiments are more representative of the conditions used by GM to coat engine cylinder bores, it was decided that it is more important to match the cylinder experiment data, i.e. the data for the coating roughness, than the flat plate experiment data. Good agreement was obtained between the calculated and measured results for the coating roughness, by reducing the droplet sizes in the over-spray found in the procedure described above by a factor of 0.2 (shown in Figure A3, labeled, 'Reduced $D_{S}$ '). The mass flux magnitudes and spray angles were not changed. The mass flux distributions shown in Figure 5 of section III.2.1 include the reduced droplet size for the overspray.
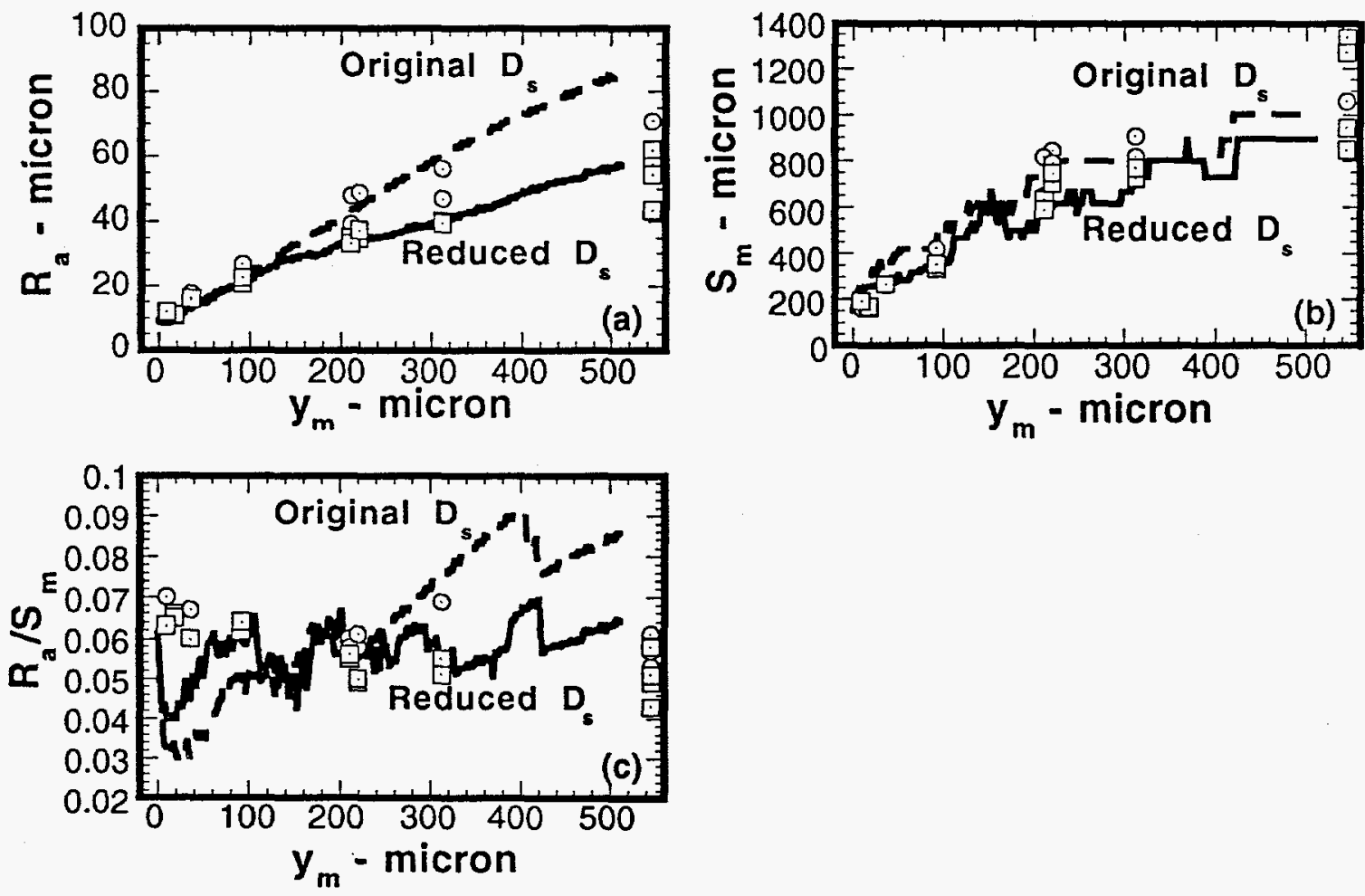

Figure A3. Comparison of calculated and measured results for the coating roughness as a function of the coating thickness. Two sets of calculated results are shown, the 'Original $\mathrm{D}_{S}$ ' results and the 'Reduced $D_{s}$ ' results, where the latter used smaller values for the droplet sizes in the over-spray portion of the mass flux than did the former. 


\section{APPENDIX B. CONVERGENCE OF THE SOLUTIONS}

The calculations were tested for convergence with respect to the numerical parameters. The numerical parameters are the number of node points used to define the coating surface, the time step, the random number sequence and the number of time steps taken between the process of searching and removing loops from the calculated coating surface. The effect of doubling the number of node points used to define the coating surface relative to the number used (3200) in most of the calculations carried out for this study is shown in Figure B1. There is good agreement between the two sets of results for the first half of the simulations, but some differences develop during the latter half of the simulations. The maximum difference is $16 \%$. Both sets of results show the characteristic change in slope at $y_{m}=120$ microns. Overall, there is a fair agreement for the magnitude and basic structure of the results for $R_{a}$ as a function of $y_{m}$.

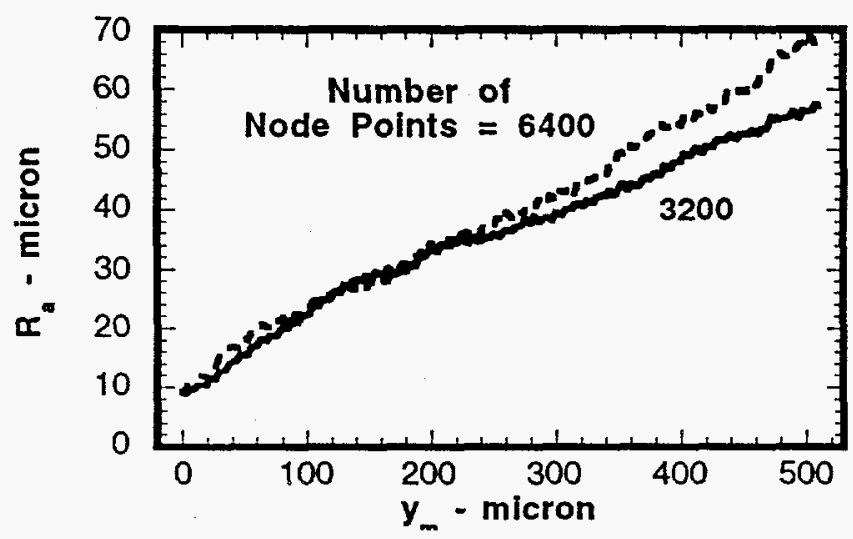

Figure B1. The effect of the number of node points on the results for $R_{\mathbf{a}}$.

The effect of the time step used to integrate Equation (1) is shown in Figure B2. The nominal value for the time step used for most of the calculations carried out for this study is $0.0002 \mathrm{~s}$. Results for the nominal value of the time step as well as a time step equal to 0.0001s are shown in the figure. The two sets of results are in very close agreement indicating the results using the nominal time step are well converged.

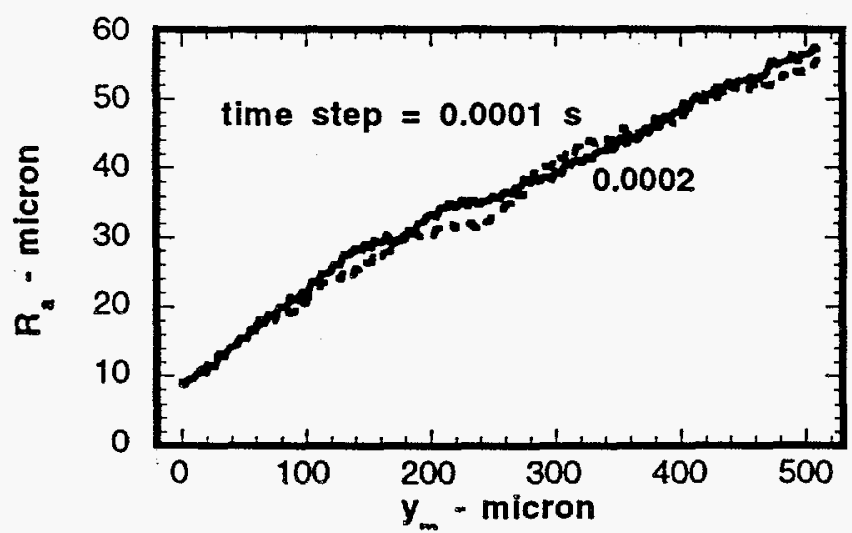

Figure B2. The effect of the time step used to integrate the governing equations on the results for $\mathrm{Ra}$. 
The effect of the random number sequence used in Equation (4) for determining the droplet impact times is shown in Figure B3. The random number sequence affects the order of the droplets deposited on the target. This order will not be important if the number of droplets deposited in the simulations is large enough such that a good statistical average effect of the stochastic deposition of droplets on the coating roughness is obtained. That is, if the number of droplets deposited is sufficiently large, the differences created by altering the order of their deposition will cancel out and the end results will be independent of the order of deposition. Typically, 22 million droplets are deposited in the simulations. Two sets of results are shown in Figure B3 which were obtained using different random number sequences. The close agreement between the two sets of results shows that the results represent a good statistical average, and are well converged with respect to the random number sequence.

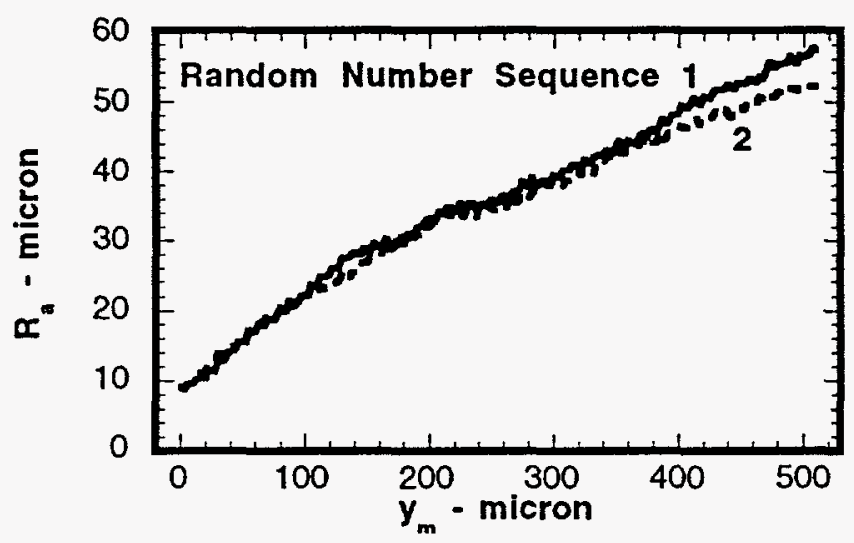

Figure B3. The effect of the random number sequence used to determine the order of the droplets deposited on the target.

The effect of the number of time steps taken, $\mathrm{N}_{\mathrm{L}}$, for each execution of the process where loops in the coating surface are searched for and removed is shown in Figure B4. Loops in the computed coating surface can occur due to the explicit integration scheme used to perform the calculations. These loops can degrade the accuracy of the calculations so it is important that they be removed while they are still small relative to the overall size of the computed coating surface. However, this process increases the CPU time for completing the calculations, so it is done as seldom as possible without compromising the accuracy of the calculations. The nominal value used for $\mathrm{N}_{\mathrm{L}}$ is five. Results using the nominal value as well as a value of one for $\mathrm{N}_{\mathrm{L}}$ are shown in Figure B4. The close agreement between the two sets of results shows that the results are well converged with respect to $\mathrm{N}_{\mathrm{L}}$. 


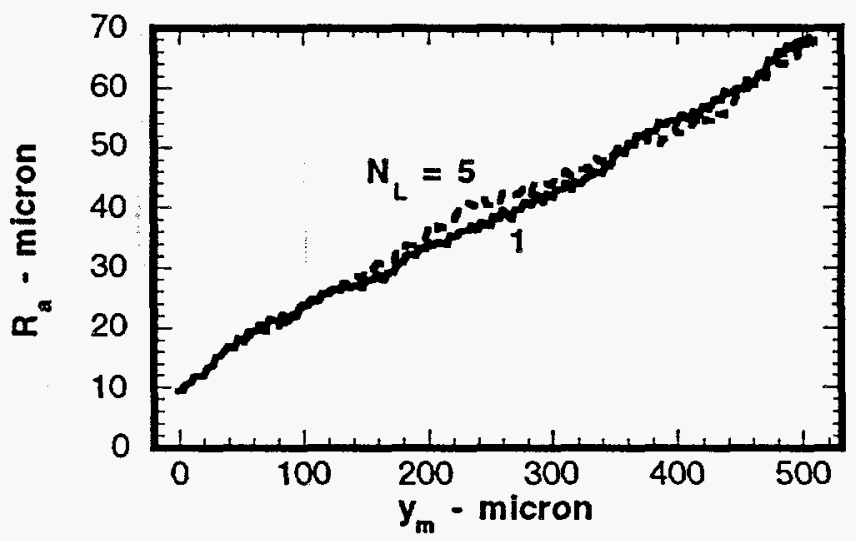

Figure B4. The effect of the number of time steps taken, $\mathrm{N}_{\mathrm{L}}$, for each execution of the process where loops in the coating surface are searched for and removed. 
INITIAL DISTRIBUTION

UNLIMITED RELEASE

North Dakota State University

Attn: Professor G. P. Bierwagen

Department of Polymers and Coatings

54 Dunbar Hall

Fargo, North Dakota 58105

University of California

Attn: Professor R. Greif

Mechanical Engineering Department

Rm 6107 Etchevery Hall

Berkeley, California 94720

University of Minnesota

Attn: Dr. I. Gates

Box 43, 151 Amundson Hall

421 Washington Avenue SE

Minneapolis, Minnesota 55455

University of Minnesota

Attn: Dr. M. Pekurovsay

Chemical Engineering and Material Science

Department

421 Washington Avenue

Minneapolis, Minnesota 55455

Abitibi-Price Inc.

Attn: B. A. Sproule

Sheridan Park

2240 Speakman Drive

Mississauga, Ontario L5K 1 A9

Anocoil Corporation

Attn: R. E. Hammond

Plate Division

P. O. Box 1318

Rockville, Connecticut 06066

Avery Dennison

Attn: Dr. L. Sartor

Research Center

2900 Bradley Street

Pasadena, California 91107-1599
BASF AG

Attn: Hans-Günter Wagner

Engineering R \& D

ZET/EA - L544

67056 Ludwigshafen

Germany

R. C. Campbell

2016 South Dexter Street

Flint, Michigan 48503-4524

Combustion Research and Flow Technology, Inc.

Attn: Dr. Neeraj Sinha

174 North Main Street

Building 3

P. O. Box 1150

Dublin, PA 18917

Danavision

Attn: R. L. Sullivan

D. V Research Sdn Bhd

Locked Bag No. 11

Nilai Industrial Estate

71800 Nilai

Negen Sembilan, Malaysia

DuPont Central Research \& Development

Attn: Dr. E. D. Cohen

Corporate Coating Technology Center

Experimental Station

P. O. Box 80328

Wilmington, DE 19880-0328

DuPont Medical Products

Attn: Dr. D. E. Jacober

Diagnostic Imaging

Research \& Development

P. O. Box 267

Brevard, NC 28712

Fasson

Attn: T. McKeon

Roll Division

3011 Independence Drive

Fort Wayne, IN 46808

FLEXcon

Attn: D. R. Miller

1 FLEXcon Industrial Park

Spencer, MA 01562-2642 
Fluent Inc.

Attn: E. W. Grald

Centerra Resource Park

10 Cavendish Court

Lebanon, NH 03766-1442

Fuji Photo Film Co., LTD.

Attn: C. Kobayashi

Miyanodai Technology Development Center

798, Miyanodai, Kaisei-Machi

Ashigarakami-Gun

Kanagawa, 258 Japan

Fuji Photo Film Co., LTD.

Attn: K. Miyamoto

Ashigara Factory

210, Nakanuma Minamiashigara-Shi

Kanagawa, 250-01 Japan

General Motors North American Operations

(2)

Attn: A. McMillan

Attn: Dr. J. N. Hall

Manufacturing Building

Mail Code: AMD-36

Mound Road 1-9

Box 9040

Warren, Michigan 48090-9040

General Motors Powertrain (2)

Attn: L. Byrnes

Attn: M. Kramer

895 Joslyn Avenue

Pontiac, Michigan 48340

General Motors Research and Development

Attn: Dr. R. E. Teets

Physics Department

30500 Mound Road

Box 9055

Warren, Michigan 48090-9055

W. L. Gore \& Associates, INC.

Attn: D. E. Hazlebeck

Fabrics Division

297 Blue Ball Road

Elkton, MD 21921

Dr. E. B. Gutoff

194 Clark Road

Brookline, MA 02146-5824
Hoechst Diafoil

Attn: F. G. Funderburk

Hood Road

P. O. Box 1400

Greer, SC 29652

International Paper

Attn: Dr. P. J. Veverka

Corporate Research Center

Long Meadow Road

Tuxedo, NY 10987

Konica Corporation

Attn: K. Kazama

No. 1 Sakura-machi Hino-shi

Tokyo 191, Japan

L.P.T.M.-C.N.R.S.

Attn: Dr. B. Prunet-Foch

4 Ter Route des Gardes

F-92190 Meudon

France

Lehrstuhl Für Strömungsmechanik

Technische Fakultät

Friedrich-Alexander-Universität

Erlangen-Nürnberg

Attn: Dr.-Ing. G. Brenn

Cauerstrasse 4

D-91058 Erlangen

Germany

Nippon Steel Corporation

Attn: H. Kanai

Technical Development Bureau

1 Kimitsu, Kimitsu-City

Chiba Pref. 299-11 Japan

Norton Company

Attn: Dr. W. P. Yang

P.O. Box 808

Troy, NY 12181-0808

Owens-Corning Science \& Technology

Center

Attn: G. Gao

2790 Columbus Road, Route 16

Granville, Ohio 43023-1200 
Philips Research

Attn: Dr. ir. J.H. Lammers

Nederlandse Philips Bedrijven B.V.

Prof. Holstlaan 4 (WB31)

5656. AA Eindhoven

The Netherlands

Polaroid Corporation

Attn: E. J. Choinski

1265 Main Street - W4

Waltham, Massachusetts 02154

Polaroid Corporation

Attn: Dr. D. Huang

1265 Main Street, W4-1H

Waltham, Massachusetts 02254

Polaroid Corporation

Attn: S. N. Najjar

1 Upland Road, Building N-2 2B

Norwood, Massachusetts 02062

Polaroid Corporation

Attn: Dr. S. X. Pan

1265 Main Street, W1-2

Waltham, Massachusetts 02154

Polaroid Corporation

Attn: R. F. Praino, Jr.

One Upland Road, Building N2-1D

Norwood, Massachusetts 02062

Polaroid Corporation

Attn: Dr. M. Sujanani

1265 Main Street, W5-2

Waltham, Massachusetts 02254

Polychrome Americas

Polychrome Corporation

Attn: Y. Maruko

160 Terminal Avenue

Clark, NJ 07066-1372

SKC Limited

Attn: K. Chang-Hwan

\#633 Chongja-1Dong, Changan-Gu.

Suwon, Kyonggi-Do, 440-300

Korea

3M Imaging Systems

Group Engineering

Attn: Dr. M. S. Carvalho

3M Center, Building 235-BF-19

St. Paul, MN 55144-1000
3M Engineering Systems and Technology

Attn: S. Fuchigami

3M Center, Building 570-1W-03

St. Paul, MN 55144-1000

Toshiba Corporation

Attn: S. Yamaguchi

70, Yanagi-Cho, Saiwai-Ku

Kawasaki, 210 Japan

United Technologies Research Center

Attn: Dr. T. J. Barber

411Silver Lane, MS 129-20

East Hartford, Connecticut 06108 


$\begin{array}{lll}\text { MS 0333 } & 1841 & \text { A. J. Hurd } \\ \text { MS 0825 } & 9115 & \text { B. Hassan } \\ \text { MS 0825 } & 9115 & \text { A. R. Lopez } \\ \text { MS 0825 } & 9115 & \text { W. L. Oberkampf } \\ \text { MS 0825 } & 9115 & \text { W. H. Rutledge } \\ \text { MS 0826 } & 9111 & \text { K. S. Chen } \\ \text { MS 0826 } & 9111 & \text { W. Hermina } \\ \text { MS 0826 } & 9111 & \text { P. R. Schunk } \\ \text { MS 0827 } & 9114 & \text { R. T. McGrath } \\ \text { MS 0828 } & 9102 & \text { R. D. Skocypec } \\ \text { MS 0835 } & 9113 & \text { R. C. Dykhuizen } \\ \text { MS 0841 } & 9100 & \text { P. J. Hommert } \\ \text { MS 1129 } & 1841 & \text { M. F. Smith } \\ \text { MS 1129 } & 1841 & \text { R. A. Neiser } \\ \text { MS 1129 } & 1841 & \text { T. J. Roemer } \\ \text { MS } 9001 & 8000 & \text { T. Hunter } \\ & & \text { Attn: J. B. Wright, 2200 } \\ & & \text { M. E. John, } 8100 \\ & & \text { L. A. West, } 8200 \\ & & \text { W. J. McLean, } 8300 \\ & & \text { R. C. Wayne, } 8400 \\ & & \text { P. N. Smith, } 8500 \\ \text { P. E. Brewer, } 8800 \\ \text { D. Crawford, } 8900\end{array}$

MS $90428742 \quad$ M. R. Birnbaum

MS 90428345 C. M. Hartwig

MS $90428345 \quad$ P. A. Spence

MS 90438743 M. P. Kanouff (10)

MS 90438743 A. R. Ortega

MS 94018700 T. M. Dyer

MS 94058743 P. E. Nielan

MS 90218815 Technical Communications Department, for OSTI (10)

MS 90218815 Technical Communications Department/Technical Library, 4414

MS $08994414 \quad$ Technical Library (4)

MS 9018 8940-2 Central Technical Files (3) 